Document downloaded from:

http://hdl.handle.net/10251/156326

This paper must be cited as:

Boiti, C.; Jornet Casanova, D.; Oliaro, A. (2019). The Gabor wave front set in spaces of ultradifferentiable functions. Monatshefte für Mathematik. 188(2):199-246.

https://doi.org/10.1007/s00605-018-1242-3

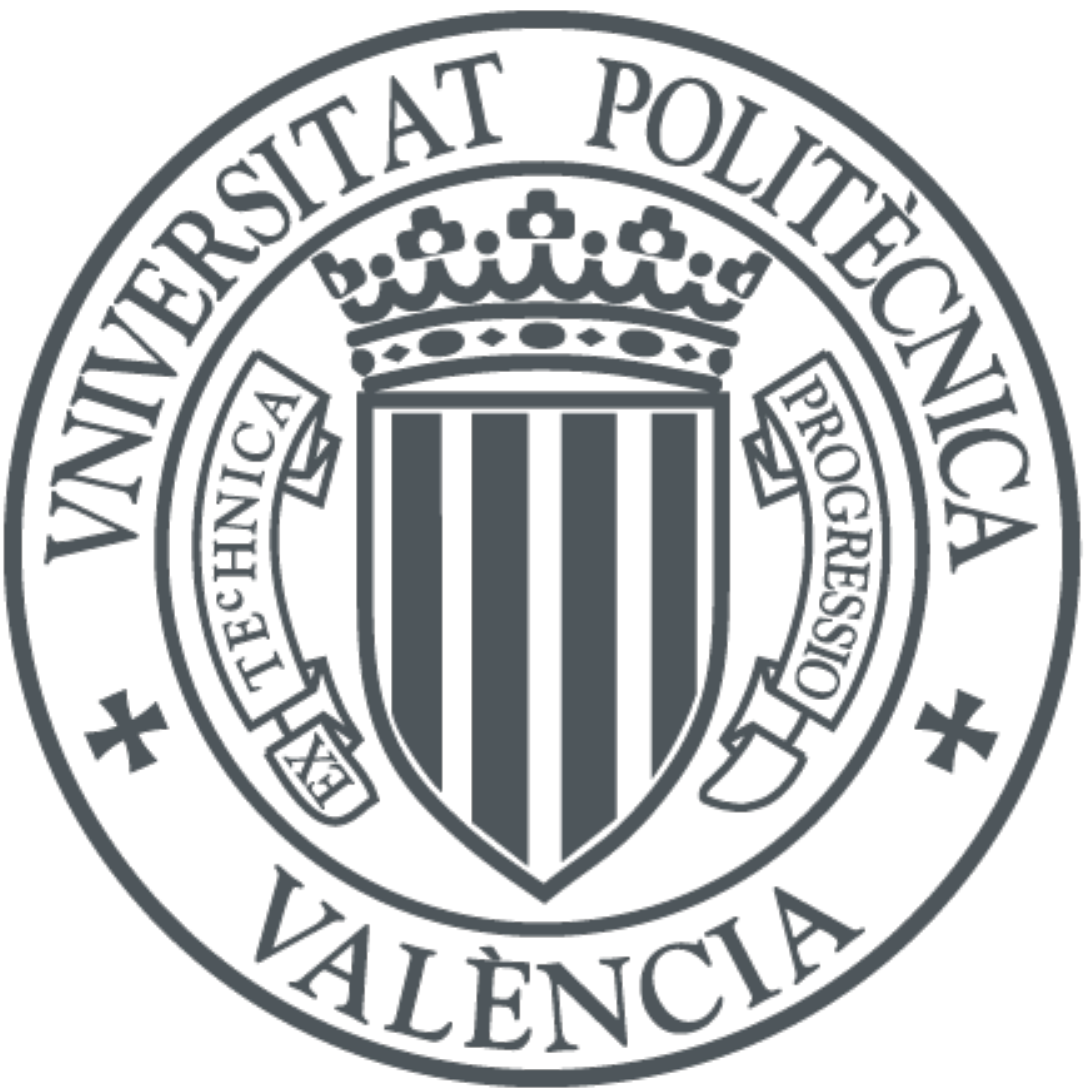

The final publication is available at

https://doi.org/10.1007/s00605-018-1242-3

Copyright Springer-Verlag

Additional Information 


\title{
THE GABOR WAVE FRONT SET IN SPACES OF ULTRADIFFERENTIABLE FUNCTIONS
}

\author{
CHIARA BOITI, DAVID JORNET, AND ALESSANDRO OLIARO
}

\begin{abstract}
We consider the spaces of ultradifferentiable functions $\mathcal{S}_{\omega}$ as introduced by Björck (and its dual $\mathcal{S}_{\omega}^{\prime}$ ) and we use time-frequency analysis to define a suitable wave front set in this setting and obtain several applications: global regularity properties of pseudodifferential operators of infinite order and the micro-pseudolocal behaviour of partial differential operators with polynomial coefficients and of localization operators with symbols of exponential growth. Moreover, we prove that the new wave front set, defined in terms of the Gabor transform, can be described using only Gabor frames. Finally, some examples show the convenience of the use of weight functions to describe more precisely the global regularity of (ultra)distributions.
\end{abstract}

\section{Introduction}

The wave front set is a basic concept in the local theory of linear partial differential operators and it extends the one of singular support of a distribution. It deals with the analysis of the singularities of a function (or distribution) and, at the same time, describes the directions along which the high frequencies (in terms of the Fourier transform) responsible for those singularities propagate. In the classical context of Schwartz distributions theory it was originally defined by Hörmander [21]. There is a huge literature on wave front sets for the study of the regularity of linear partial differential operators in spaces of distributions or ultradistributions in a local sense; see, for instance, $[21,23,24,25,34,15,1,2,35,7,6]$ and the references therein.

In global classes of functions and distributions (like the Schwartz class $\mathcal{S}$ and its dual) the concept of singular support does not make sense, since we require the information on the whole $\mathbb{R}^{d}$. However, we still can define a global wave front set to describe the micro-regularity of a distribution, where the cones are taken with respect to the whole of the phase space variables. In fact, in [22] Hörmander introduced two different types of global wave front sets: the $C^{\infty}$ wave font set, in the Beurling setting, for temperate distributions $u \in \mathcal{S}^{\prime}$, and the analytic wave front set, in the Roumieu setting, for ultradistributions $\mathcal{S}_{A}^{\prime}$ of Gelfand-Shilov type, addressed to the study of quadratic hyperbolic operators. Unfortunately, these global versions of wave front set have been almost ignored in the literature, whereas they will represent the key point of our discussion. Only very recently, Rodino and Wahlberg [35] recovered the concept of $C^{\infty}$ wave front set of [22] and showed that it can be reformulated in terms of the short-time Fourier transform, which treats simultaneously the variables and covariables of a function (or distribution) in order to quantify the energy of a signal at some time $x_{0}$ and some frequency $\xi_{0}$. Since the wave front set has to do with a simultaneous analysis of points (variables) and

2010 Mathematics Subject Classification. Primary 35A18; Secondary 46F05, 42C15, 35S05.

Key words and phrases. Gabor wave front set, weighted Schwartz classes, short-time Fourier transform, Gabor frames. 
directions (covariables), it is very natural to try to apply methods of time-frequency analysis in connection with the wave front set. Indeed, in [35] the authors use this advantage to show also that the original wave front set introduced by Hörmander can be described merely with the information given by a Gabor frame, which is a fundamental tool in the theory of timefrequency analysis with applications in signal processing and related issues in function space theory and numerical analysis. Besides, recent applications of Gabor frames concern also the analysis of partial differential equations and pseudodifferential equations (see the references quoted in the introduction of [35] for more information). On the other hand, Nakamura [29] introduces the homogenous wave front set for the study of propagation of micro-singularities for Schrödinger equations, and it turns out to be equal to the Gabor wave front set [37]. Cappiello and Schulz [12] recover the analytic wave front set of [22], defined in terms of a very general known version of the FBI transform as introduced originally by Sjöstrand [39], show that it can be written using the Gabor transform (with Gaussian window) and study some cases not treated by Hörmander for Gelfand-Shilov ultradistributions of Gevrey type.

The modern theory of general linear PDEs has been largely addressed to local problems, i.e., to the study of solutions in a suitable small neighbourhood of a point in $\mathbb{R}^{d}$. More recently, several authors have considered the study of (pseudo)differential operators from a global point of view; see, for example $[22,30,29,37]$. The Fourier transform and pseudo-differential calculus find in $\mathbb{R}^{d}$ their natural setting. In fact, some problems in Quantum Mechanics, Signal Analysis and other applications in Physics and Engineering are represented by the study of solutions in the whole Euclidean space $\mathbb{R}^{d}$. Motivated by these connections, the theory of timefrequency analysis has become a very suitable tool for a better understanding of the study of (pseudo)differential operators in the global setting and, in particular, in the Schwartz class $\mathcal{S}$ (see [35]) or in Gelfand-Shilov spaces of Gevrey type (see [12]).

In the present paper we work in the classes of ultradifferentiable functions $\mathcal{S}_{\omega}\left(\mathbb{R}^{d}\right)$, where $\omega$ is a weight function in the sense of Braun, Meise and Taylor [11], which we assume to be also subadditive, in order to have a consistent definition of modulation spaces given by exponential weights. Hence, we recover in particular the classes as introduced by Björck [3], with the difference that we impose that the composition of the weight and the exponential is convex, which allows the use of convex analysis techniques. The classes under consideration are suitable for our purposes, since they are invariant under Fourier transform and provide a big scale of spaces that contain as a particular case the Schwartz class when the weight function is $\omega(t)=\log (1+t), t>0$. We have seen in the literature the benefits of time-frequency analysis when applied to such classes (see [19]), even in combination with the global theory of (pseudo)differential operators (see e.g. the paper by the same authors [8] and the references therein, or $[32,33]$ when the classes are defined by sequences in the sense of Denjoy-Carleman; see [27] for a detailed study of the structure of these spaces when defined by sequences). We have to mention also that our classes always contain compactly supported functions (they are non-quasianalytic) and we recover Gelfand-Shilov spaces of Beurling type of index $s>1$ when the weight function is $\omega(t)=t^{1 / s}$ (i.e. a Gevrey weight).

The purpose of our paper is to define the Beurling version of the analytic wave front set found in $[22,12]$ (where the authors only treated the Roumieu case) in the setting of $\mathcal{S}_{\omega^{-}}^{\prime}$ ultradistributions, show that it can be described in terms of Gabor frames (as it is done in the setting of temperate distributions in [35]) and apply it to the study of the global regularity 
of (pseudo)differential operators of infinite order (in [35] the authors cannot treat operators of infinite order, since they have symbols with polynomial growth). So, we extend, among other results, part of the work [35] to the ultradifferentiable setting and treat the Beurling case, which is new in the literature (the authors in $[22,12]$ treat the Roumieu case only for Gelfand-Shilov ultradistributions).

From [19], we know that a function $f \in \mathcal{S}_{\omega}\left(\mathbb{R}^{d}\right)$ can be characterized in terms of the growth of its Gabor transform, i.e. of its short-time Fourier transform. We use this fact to extend to the ultradifferentiable setting some known properties of the Gabor transform in the frame of the Schwartz class $\mathcal{S}$, that we could not find in the literature for $\mathcal{S}_{\omega}$, and we add them here for the reader's convenience (see Section 2).

In Section 3 we consider the global $\omega$-wave front set $\mathrm{WF}_{\omega}^{\prime}(u)$, for $\omega$-tempered distributions $u \in \mathcal{S}_{\omega}^{\prime}\left(\mathbb{R}^{d}\right)$, defined as the complement of the points $z_{0} \in \mathbb{R}^{2 d} \backslash\{0\}$ for which there exists an open conic set $\Gamma$ containing $z_{0}$ such that

$$
\sup _{z \in \Gamma} e^{\lambda \omega(z)}\left|V_{\varphi} u(z)\right|<+\infty, \quad \forall \lambda>0
$$

where $V_{\varphi} u$ is the Gabor transform of $u$ with respect to the window $\varphi \in \mathcal{S}_{\omega}\left(\mathbb{R}^{d}\right)$ (we prove that the definition does not depend on the choice of $\varphi$ ). This definition of wave front set seems natural since the Gabor transform allows to analyze simultaneously the ultradistribution with respect to variables and covariables.

However, in many applications to signal processing and related topics, often Gabor frames come out to be the most appropriate tool (see, for instance, $[14,20]$ ). For this reason it is also useful to consider a Gabor $\omega$-wave front set $\mathrm{WF}_{\omega}^{G}(u)$, defined in terms of the decay of the Gabor coefficients $\langle u, \Pi(\lambda) \varphi\rangle$ of the ultradistribution $u \in \mathcal{S}_{\omega}^{\prime}\left(\mathbb{R}^{d}\right)$ (see Definition 3.3), where $\Pi(\lambda) \varphi(y)=e^{i\left\langle y, \lambda_{2}\right\rangle} \varphi\left(y-\lambda_{1}\right)$ with $\lambda=\left(\lambda_{1}, \lambda_{2}\right)$ in a suitable lattice $\Lambda$. Actually this is equivalent to analyze the decay of the Gabor transform of $u$ on a conical set intersected with $\Lambda$, so that it is natural to study the relation between these two wave front sets. One of the main results of this paper, Theorem 3.17, is that $\mathrm{WF}_{\omega}^{\prime}(u)=\mathrm{WF}_{\omega}^{G}(u)$ for all $u \in \mathcal{S}_{\omega}^{\prime}\left(\mathbb{R}^{d}\right)$, if the lattice is sufficiently dense. In the particular case of $\omega(t)=\log (1+t)$ we recover the results of [35] about wave front sets of tempered distributions.

In order to examine Gabor $\omega$-wave front sets, we need suitable modulation spaces with exponential weights, in the setting of $\omega$-ultradistributions. To this aim we prove in Section 3 those results about modulation spaces which differ from the classical ones (cf. e.g. [18]). Moreover, we prove two natural properties for the Gabor $\omega$-wave front set. Namely, for an ultradistribution $u \in \mathcal{S}_{\omega}^{\prime}\left(\mathbb{R}^{d}\right)$, we show that $\mathrm{WF}_{\omega}^{\prime}(u)$ is empty if and only if $u \in \mathcal{S}_{\omega}\left(\mathbb{R}^{d}\right)$, and that it is not affected by translations and modulations (time-frequency shifts), as expected in the global setting.

In Section 4 our results in the former sections are applied to study the global regularity of some kind of pseudodifferential operators of infinite order with our global wave front set. For a global symbol $a(x, \xi)$ with exponential growth in the second variable, defined in the spirit of [15] (see Definition 4.2), we consider the Kohn-Nirenberg quantization

$$
a(x, D) f(x):=(2 \pi)^{-d} \int_{\mathbb{R}^{d}} e^{i\langle x, \xi\rangle} a(x, \xi) \widehat{f}(\xi) d \xi
$$


which is well defined for $f \in \mathcal{S}_{\omega}\left(\mathbb{R}^{d}\right)$. We analyze the kernel of the Gabor transform of this pseudo-differential operator to prove that

$$
\mathrm{WF}_{\omega}^{\prime}(a(x, D) u) \subseteq \text { conesupp }(a)
$$

where cone $\operatorname{supp}(a)$ is the conic support of $a(x, \xi)$, as defined in [22] (see also Definition 4.10). As far as we know, this is new in the literature. As a consequence, we have that the KohnNirenberg quantization $a(x, D)$, for a symbol $a(x, \xi) \in \mathcal{S}_{\omega}\left(\mathbb{R}^{2 d}\right)$ with compact support, is a globally $\omega$-regularizing pseudo-differential operator, in the sense that for every $u \in \mathcal{S}_{\omega}^{\prime}\left(\mathbb{R}^{d}\right)$ we have that $a(x, D) u \in \mathcal{S}_{\omega}\left(\mathbb{R}^{d}\right)$.

We also study the micro-pseudolocal behaviour of a linear partial differential operator with polynomial coefficients using purely the properties of the Gabor transform (Proposition 4.13) and also of a very general type of localization operators (Theorem 4.15), obtaining in the Beurling setting the analogous result of [12, Proposition 3.3]. Finally, in Section 5 we calculate the wave front set of some concrete ultradistributions and show, in particular, the usefulness of working with different weight functions, as in Example 5.4, where we analyze the global $\omega$-wave front set of some ultradistributions for different weight functions $\omega$.

\section{Preliminaries and the short-time Fourier transform in $\mathcal{S}_{\omega}\left(\mathbb{R}^{d}\right)$}

Given a function $f \in L^{1}\left(\mathbb{R}^{d}\right)$, the Fourier transform of $f$ is defined as

$$
\mathcal{F}(f)=\hat{f}(\xi)=\int_{\mathbb{R}^{d}} e^{-i\langle x, \xi\rangle} f(x) d x,
$$

with standard extensions to more general spaces of functions and distributions.

Definition 2.1. A non-quasianalytic subadditive weight function is a continuous increasing function $\omega:[0,+\infty) \rightarrow[0,+\infty)$ satisfying the following properties:

$(\alpha) \omega\left(t_{1}+t_{2}\right) \leq \omega\left(t_{1}\right)+\omega\left(t_{2}\right) \quad \forall t_{1}, t_{2} \geq 0 ;$

(ß) $\int_{1}^{+\infty} \frac{\omega(t)}{t^{2}} d t<+\infty$;

$(\gamma) \exists a \in \mathbb{R}, b>0$ s.t. $\quad \omega(t) \geq a+b \log (1+t) \quad \forall t \geq 0$;

( $\delta) \varphi_{\omega}(t):=\omega\left(e^{t}\right)$ is convex.

We then define $\omega(\zeta):=\omega(|\zeta|)$ for $\zeta \in \mathbb{C}^{d}$.

We denote by $\varphi_{\omega}^{*}$ the Young conjugate of $\varphi_{\omega}$, defined by

$$
\varphi_{\omega}^{*}(s):=\sup _{t \geq 0}\left\{s t-\varphi_{\omega}(t)\right\}, \quad s \geq 0 .
$$

Note that $\varphi_{\omega}^{*}$ is increasing and convex, and $\varphi_{\omega}^{* *}=\varphi_{\omega}$ by Fenchel-Moreau Theorem (see for example [10]). Moreover, $\varphi_{\omega}^{*}(s) / s$ is increasing since

$$
\varphi_{\omega}^{*}(0)=\sup _{t \geq 0}\left(-\varphi_{\omega}(t)\right) \leq 0
$$


and therefore, for $0<s_{1}<s_{2}$, by the convexity of $\varphi_{\omega}^{*}$ :

$$
\varphi_{\omega}^{*}\left(s_{1}\right)=\varphi_{\omega}^{*}\left(\frac{s_{1}}{s_{2}} s_{2}+\left(1-\frac{s_{1}}{s_{2}}\right) 0\right) \leq \frac{s_{1}}{s_{2}} \varphi_{\omega}^{*}\left(s_{2}\right)+\left(1-\frac{s_{1}}{s_{2}}\right) \varphi_{\omega}^{*}(0) \leq \frac{s_{1}}{s_{2}} \varphi_{\omega}^{*}\left(s_{2}\right) .
$$

It will be also useful in the sequel the following inequality

$$
2^{j} e^{-\lambda \varphi_{\omega}^{*}\left(\frac{j}{\lambda}\right)} \leq e^{-3 \lambda \varphi_{\omega}^{*}\left(\frac{j}{3 \lambda}\right)}, \quad \forall j \in \mathbb{N}_{0}, \lambda>0 .
$$

Estimates of this kind are well known (see, for instance, $[11,5]$ ), usually stated under slightly different conditions on $\omega$. We give here a short proof of (2.1) for the sake of completeness. By definition of $\varphi_{\omega}^{*}$ for $\varphi_{\omega}(t)=\omega\left(e^{t}\right)$ and by the subadditivity of $\omega$ :

$$
\begin{aligned}
\varphi_{\omega}^{*}(s) & =\sup _{t \geq 0}\left\{t s-\varphi_{\omega}(t)\right\} \geq \sup _{t \geq 1}\left\{t s-\varphi_{\omega}(t)\right\}=\sup _{\sigma \geq 0}\left\{(\sigma+1) s-\varphi_{\omega}(\sigma+1)\right\} \\
& =s+\sup _{\sigma \geq 0}\left\{\sigma s-\omega\left(e e^{\sigma}\right)\right\} \geq s+\sup _{\sigma \geq 0}\left\{\sigma s-3 \varphi_{\omega}(\sigma)\right\}=s+3 \varphi_{\omega}^{*}\left(\frac{s}{3}\right) .
\end{aligned}
$$

Therefore, for $s=j / \lambda$ and multiplying by $\lambda$ :

$$
\lambda \varphi_{\omega}^{*}\left(\frac{j}{\lambda}\right) \geq j+3 \lambda \varphi_{\omega}^{*}\left(\frac{j}{3 \lambda}\right)
$$

and hence

$$
2^{j} e^{3 \lambda \varphi_{\omega}^{*}\left(\frac{j}{3 \lambda}\right)} \leq e^{\lambda \varphi_{\omega}^{*}\left(\frac{j}{\lambda}\right)} .
$$

Definition 2.2. We define $\mathcal{S}_{\omega}\left(\mathbb{R}^{d}\right)$ as the set of all $u \in \mathcal{S}\left(\mathbb{R}^{d}\right)$ such that

(i) $\forall \lambda>0, \alpha \in \mathbb{N}_{0}^{d}: \sup _{\mathbb{R}^{d}} e^{\lambda \omega(x)}\left|D^{\alpha} u(x)\right|<+\infty$,

(ii) $\forall \lambda>0, \alpha \in \mathbb{N}_{0}^{d}: \sup _{\mathbb{R}^{d}} e^{\lambda \omega(\xi)}\left|D^{\alpha} \widehat{u}(\xi)\right|<+\infty$,

where $\mathbb{N}_{0}:=\mathbb{N} \cup\{0\}$ and $D^{\alpha}=(-i)^{|\alpha|} \partial^{\alpha}$.

As usual, the corresponding dual space is denoted by $\mathcal{S}_{\omega}^{\prime}\left(\mathbb{R}^{d}\right)$ and is the set of all linear and continuous functionals $u: \mathcal{S}_{\omega}\left(\mathbb{R}^{d}\right) \rightarrow \mathbb{C}$. An element of $\mathcal{S}_{\omega}^{\prime}\left(\mathbb{R}^{d}\right)$ is called an $\omega$-tempered distribution.

In $\left[8\right.$, Thm. 4.8] we provided the space $\mathcal{S}_{\omega}\left(\mathbb{R}^{d}\right)$ with different equivalent systems of seminorms. For example, for $u \in \mathcal{S}_{\omega}\left(\mathbb{R}^{d}\right)$, the family of seminorms

$$
p_{\lambda, \mu}(u):=\sup _{\alpha, \beta \in \mathbb{N}_{0}^{d}} \sup _{x \in \mathbb{R}^{d}}\left|x^{\beta} D^{\alpha} u(x)\right| e^{-\lambda \varphi_{\omega}^{*}\left(\frac{|\alpha|}{\lambda}\right)-\mu \varphi_{\omega}^{*}\left(\frac{|\beta|}{\mu}\right)},
$$

for $\lambda, \mu>0$. On the other hand, it is not difficult to see (using, for instance, [8, Lemma 4.7(ii)]) that the family of seminorms

$$
q_{\lambda, \mu}(u):=\sup _{\alpha \in \mathbb{N}_{0}^{d}} \sup _{x \in \mathbb{R}^{d}}\left|D^{\alpha} u(x)\right| e^{-\lambda \varphi_{\omega}^{*}\left(\frac{|\alpha|}{\lambda}\right)+\mu \omega(x)}, \quad \lambda, \mu>0,
$$

defines another equivalent system of seminorms for $\mathcal{S}_{\omega}\left(\mathbb{R}^{d}\right)$.

We recall that $\mathcal{S}_{\omega}\left(\mathbb{R}^{d}\right) \subseteq \mathcal{S}\left(\mathbb{R}^{d}\right)$ and for their correspondent dual spaces we have the inclusion $\mathcal{S}^{\prime}\left(\mathbb{R}^{d}\right) \subseteq \mathcal{S}_{\omega}^{\prime}\left(\mathbb{R}^{d}\right)$. Moreover, the Fourier transform is a continuous automorphism from $\mathcal{S}_{\omega}\left(\mathbb{R}^{d}\right)$ to $\mathcal{S}_{\omega}\left(\mathbb{R}^{d}\right)$ and from $\mathcal{S}_{\omega}^{\prime}\left(\mathbb{R}^{d}\right)$ to $\mathcal{S}_{\omega}^{\prime}\left(\mathbb{R}^{d}\right)$. 
The condition $(\beta)$ of non-quasianalyticity in Definition 2.1 ensures the existence of functions with compact support in $\mathcal{S}_{\omega}\left(\mathbb{R}^{d}\right)$. To be more precise, let us briefly recall (see $[11,4]$ ) the definition of the space $\mathcal{E}_{(\omega)}(\Omega)$ of $\omega$-ultradifferentiable functions of Beurling type in an open subset $\Omega$ of $\mathbb{R}^{d}$. It is the set

$$
\begin{aligned}
\mathcal{E}_{(\omega)}(\Omega):=\left\{f \in C^{\infty}(\Omega):\right. & \forall K \subset \subset \Omega, \forall m \in \mathbb{N} \\
& \left.\sup _{\alpha \in \mathbb{N}^{d}} \sup _{x \in K}\left|D^{\alpha} f(x)\right| e^{-m \varphi_{\omega}^{*}\left(\frac{|\alpha|}{m}\right)}<+\infty\right\} .
\end{aligned}
$$

To define then the space of $\omega$-ultradifferentiable functions of Beurling type with compact support, we first consider, for a compact set $K \subset \Omega$,

$$
\mathcal{D}_{(\omega)}(K):=\left\{f \in \mathcal{E}_{(\omega)}(\Omega): \operatorname{supp} f \subseteq K\right\} .
$$

This space is not trivial because of $(\beta)$ of Definition 2.1. Finally, we set the space of test functions as follows

$$
\mathcal{D}_{(\omega)}(\Omega)=\operatorname{ind}_{K} \lim _{\nearrow \Omega} \mathcal{D}_{(\omega)}(K) .
$$

Then the following continuous inclusions hold (see [17, 3]):

$$
\mathcal{D}_{(\omega)}\left(\mathbb{R}^{d}\right) \subset \mathcal{S}_{\omega}\left(\mathbb{R}^{d}\right) \subset \mathcal{E}_{(\omega)}\left(\mathbb{R}^{d}\right) .
$$

Example 2.3. An example of non-quasianalytic subadditive weight function is

$$
\omega(t)=t^{1 / s}, \quad s>1 .
$$

In this case $\mathcal{E}_{(\omega)}(\Omega)$ is the space $\gamma^{(s)}(\Omega)$ of small Gevrey functions (see [24]), $\mathcal{D}_{(\omega)}(\Omega)$ is the space of small Gevrey functions with compact support. The space $\mathcal{S}_{\omega}\left(\mathbb{R}^{d}\right)$ is the Gelfand-Shilov space of Beurling type $\Sigma_{s}\left(\mathbb{R}^{d}\right)$ (see $[31]$ ).

Other examples of admissible weights are given by

$$
\omega(t)=\log ^{\beta}\left(e^{\beta-1}+t\right), \quad \beta \geq 1 .
$$

In this case we recover, for $\beta=1$, the class $\mathcal{E}(\Omega)$ of $C^{\infty}$ functions, the class of $\mathcal{D}(\Omega)$ of $C^{\infty}$ functions with compact support in $\Omega$ and, for $\mathcal{S}_{\omega}\left(\mathbb{R}^{d}\right)$, the classical space $\mathcal{S}\left(\mathbb{R}^{d}\right)$ of rapidly decreasing functions in $\mathbb{R}^{d}$.

We refer, for instance, to [11, 1, 2] for more examples. We also refer to [9] for the comparison of the spaces $\mathcal{E}_{(\omega)}, \mathcal{D}_{(\omega)}$ with the analogous ones defined by sequences in the sense of DenjoyCarleman (in the Roumieu case as well; see at the beginning of Section 4 for more information).

Let us denote by $T_{x}, M_{\xi}$ and $\Pi(z)$, respectively, the translation, the modulation and the phase-space shift operators, defined by

$$
T_{x} f(y)=f(y-x), \quad M_{\xi} f(y)=e^{i\langle y, \xi\rangle} f(y), \quad \Pi(z) f(y)=M_{\xi} T_{x} f(y)=e^{i\langle y, \xi\rangle} f(y-x),
$$

for $x, y, \xi \in \mathbb{R}^{d}$ and $z=(x, \xi)$.

Definition 2.4. For a window function $\varphi \in \mathcal{S}_{\omega}\left(\mathbb{R}^{d}\right) \backslash\{0\}$, the short-time Fourier transform (briefly STFT) of $f \in \mathcal{S}_{\omega}^{\prime}\left(\mathbb{R}^{d}\right)$ is defined, for $z=(x, \xi) \in \mathbb{R}^{2 d}$, by:

$$
\begin{aligned}
V_{\varphi} f(z) & :=\langle f, \Pi(z) \varphi\rangle \\
& =\int_{\mathbb{R}^{d}} f(y) \overline{\varphi(y-x)} e^{-i\langle y, \xi\rangle} d y,
\end{aligned}
$$


where the bracket $\langle\cdot, \cdot\rangle$ in (2.5) and the integral in (2.6) denote the conjugate linear action of $\mathcal{S}_{\omega}^{\prime}$ on $\mathcal{S}_{\omega}$, consistent with the inner product $\langle\cdot, \cdot\rangle_{L^{2}}$.

By [19, Lemma 1.1], for $f, \varphi, \psi \in \mathcal{S}_{\omega}\left(\mathbb{R}^{d}\right)$ we have the following inversion formula:

$$
\langle\psi, \varphi\rangle f(y)=\frac{1}{(2 \pi)^{d}} \int_{\mathbb{R}^{2 d}} V_{\varphi} f(z)(\Pi(z) \psi)(y) d z .
$$

In particular, for $\psi=\varphi \in \mathcal{S}_{\omega}\left(\mathbb{R}^{d}\right) \backslash\{0\}$ :

$$
f(y)=\frac{1}{(2 \pi)^{d}\|\varphi\|_{L^{2}}^{2}} \int_{\mathbb{R}^{2 d}} V_{\varphi} f(z)(\Pi(z) \varphi)(y) d z .
$$

We recall, from [19], the following results:

Theorem 2.5. Let $\varphi \in \mathcal{S}_{\omega}\left(\mathbb{R}^{d}\right) \backslash\{0\}$ and $f \in \mathcal{S}_{\omega}^{\prime}\left(\mathbb{R}^{d}\right)$. Then $V_{\varphi} f$ is continuous and there are constants $c, \lambda>0$ such that

$$
\left|V_{\varphi} f(z)\right| \leq c e^{\lambda \omega(z)} \quad \forall z \in \mathbb{R}^{2 d}
$$

Proposition 2.6. Let $\varphi \in \mathcal{S}_{\omega}\left(\mathbb{R}^{d}\right) \backslash\{0\}$ and assume that $F: \mathbb{R}^{2 d} \rightarrow \mathbb{C}$ is a measurable function that satisfies that for all $\lambda>0$ there is a constant $C_{\lambda}>0$ such that

$$
|F(z)| \leq C_{\lambda} e^{-\lambda \omega(z)} \quad \forall z \in \mathbb{R}^{2 d} .
$$

Then

$$
f(y):=\int_{\mathbb{R}^{2 d}} F(z)(\Pi(z) \varphi)(y) d z
$$

defines a function $f \in \mathcal{S}_{\omega}\left(\mathbb{R}^{d}\right)$.

Theorem 2.7. Let $\varphi \in \mathcal{S}_{\omega}\left(\mathbb{R}^{d}\right) \backslash\{0\}$. Then, for $f \in \mathcal{S}_{\omega}^{\prime}\left(\mathbb{R}^{d}\right)$, the following are equivalent:

(i) $f \in \mathcal{S}_{\omega}\left(\mathbb{R}^{d}\right)$;

(ii) for all $\lambda>0$ there exists $C_{\lambda}>0$ such that

$$
\left|V_{\varphi} f(z)\right| \leq C_{\lambda} e^{-\lambda \omega(z)} \quad \forall z \in \mathbb{R}^{2 d}
$$

(iii) $V_{\varphi} f \in \mathcal{S}_{\omega}\left(\mathbb{R}^{2 d}\right)$.

The following lemma is well known for functions in $\mathcal{S}\left(\mathbb{R}^{d}\right)$, and hence in $\mathcal{S}_{\omega}\left(\mathbb{R}^{d}\right)$. So we omit its proof.

Lemma 2.8. For $f, \varphi \in \mathcal{S}_{\omega}\left(\mathbb{R}^{d}\right)$ we have that

$$
\widehat{V_{\varphi} f}(\eta, y)=(2 \pi)^{d} e^{i\langle\eta, y\rangle} f(-y) \overline{\widehat{\varphi}(\eta)} \quad \forall(\eta, y) \in \mathbb{R}^{2 d} .
$$

As a consequence, we can deduce the following result.

Proposition 2.9. Let $\varphi \in \mathcal{S}_{\omega}\left(\mathbb{R}^{d}\right) \backslash\{0\}$. Then

$$
V_{\varphi}: \mathcal{S}_{\omega}\left(\mathbb{R}^{d}\right) \longrightarrow \mathcal{S}_{\omega}\left(\mathbb{R}^{2 d}\right)
$$

is continuous. 
Proof. Let us first remark that if $f \in \mathcal{S}_{\omega}\left(\mathbb{R}^{d}\right)$ then $V_{\varphi} f \in \mathcal{S}_{\omega}\left(\mathbb{R}^{2 d}\right)$ by Theorem 2.7.

Since $\mathcal{S}_{\omega}$ is a Fréchet space, to prove the continuity of $V_{\varphi}$ we consider a sequence $\left\{f_{n}\right\}_{n \in \mathbb{N}} \subset$ $\mathcal{S}_{\omega}\left(\mathbb{R}^{d}\right)$ such that

$$
f_{n} \longrightarrow f \in \mathcal{S}_{\omega}\left(\mathbb{R}^{d}\right) \quad \text { in } \mathcal{S}_{\omega}\left(\mathbb{R}^{d}\right)
$$

and prove that $V_{\varphi} f_{n} \rightarrow V_{\varphi} f$ in $\mathcal{S}_{\omega}\left(\mathbb{R}^{2 d}\right)$.

Indeed, (2.10) implies that

$$
e^{i\langle\eta, y\rangle} f_{n}(-y) \overline{\widehat{\varphi}(\eta)} \longrightarrow e^{i\langle\eta, y\rangle} f(-y) \overline{\widehat{\varphi}(\eta)} \quad \text { in } \mathcal{S}_{\omega}\left(\mathbb{R}^{2 d}\right)
$$

and hence, by Lemma 2.8,

$$
\widehat{V_{\varphi} f_{n}} \rightarrow \widehat{V_{\varphi} f} \quad \text { in } \mathcal{S}_{\omega}\left(\mathbb{R}^{2 d}\right) \text {. }
$$

Applying the inverse Fourier transform, which is continuous on $\mathcal{S}_{\omega}$, we have that

$$
V_{\varphi} f_{n} \rightarrow V_{\varphi} f \quad \text { in } \mathcal{S}_{\omega}\left(\mathbb{R}^{2 d}\right)
$$

and the proof is complete.

The short-time Fourier transform also provides a new equivalent system of seminorms for $\mathcal{S}_{\omega}\left(\mathbb{R}^{d}\right)$.

Proposition 2.10. If $\varphi \in \mathcal{S}_{\omega}\left(\mathbb{R}^{d}\right) \backslash\{0\}$, then the collection of seminorms

$$
\left\|V_{\varphi} f\right\|_{\omega, \lambda}:=\sup _{z \in \mathbb{R}^{2 d}}\left|V_{\varphi} f(z)\right| e^{\lambda \omega(z)}
$$

for $\lambda>0$, forms an equivalent system of seminorms for $\mathcal{S}_{\omega}\left(\mathbb{R}^{d}\right)$.

Proof. Set

$$
\tilde{\mathcal{S}}_{\omega}\left(\mathbb{R}^{d}\right):=\left\{f \in \mathcal{S}\left(\mathbb{R}^{d}\right):\left\|V_{\varphi} f\right\|_{\omega, \lambda}<+\infty \forall \lambda>0\right\} .
$$

By Theorem 2.7 the sets $\tilde{\mathcal{S}}_{\omega}\left(\mathbb{R}^{d}\right)$ and $\mathcal{S}_{\omega}\left(\mathbb{R}^{d}\right)$ are equal. We have to prove that they have the same topology.

By the inversion formula (2.8) we have that, for $z=(x, \xi) \in \mathbb{R}^{2 d}$ and $\lambda, \mu>0$,

$$
\begin{aligned}
& e^{-\lambda \varphi_{\omega}^{*}\left(\frac{|\alpha|}{\lambda}\right)} e^{-\mu \varphi_{\omega}^{*}\left(\frac{|\beta|}{\mu}\right)}\left|y^{\beta} D_{y}^{\alpha} f(y)\right| \\
& \leq C e^{-\lambda \varphi_{\omega}^{*}\left(\frac{|\alpha|}{\lambda}\right)} e^{-\mu \varphi_{\omega}^{*}\left(\frac{|\beta|}{\mu}\right)} \int_{\mathbb{R}^{2 d}}\left|V_{\varphi} f(z)\right| \cdot\left|y^{\beta} D_{y}^{\alpha}(\Pi(z) \varphi)(y)\right| d z \\
& =C e^{-\lambda \varphi_{\omega}^{*}\left(\frac{|\alpha|}{\lambda}\right)} e^{-\mu \varphi_{\omega}^{*}\left(\frac{|\beta|}{\mu}\right)} \int_{\mathbb{R}^{2 d}}\left|V_{\varphi} f(x, \xi)\right| \cdot\left|y^{\beta} D_{y}^{\alpha} e^{i\langle y, \xi\rangle} \varphi(y-x)\right| d x d \xi \\
& \leq C \sum_{\gamma \leq \alpha}\left(\begin{array}{c}
\alpha \\
\gamma
\end{array}\right) 2^{-|\alpha|} \int_{\mathbb{R}^{2 d}}\left|V_{\varphi} f(x, \xi)\right| \cdot|y|^{|\beta|} e^{-\mu \varphi_{\omega}^{*}\left(\frac{|\beta|}{\mu}\right)} \\
& \cdot|\xi|^{|\alpha-\gamma|}\left|D_{y}^{\gamma} \varphi(y-x)\right| e^{-\lambda \varphi_{\omega}^{*}\left(\frac{|\alpha|}{\lambda}\right)} 2^{|\alpha|} d x d \xi
\end{aligned}
$$

for some $C>0$.

We shall now need the following inequality

$$
t^{j} e^{-\mu \varphi_{\omega}^{*}\left(\frac{j}{\mu}\right)} \leq C_{\mu} e^{\mu \omega(t)}, \quad \forall t>0, j \in \mathbb{N}_{0},
$$


that is well known for $t \geq 1$ with $C_{\mu}=1$ (see, for instance, [8, Lemma 4.7(i)] or [15]), and is trivial for $0<t \leq 1$ with $C_{\mu}=e^{-\mu \varphi_{\omega}^{*}(0)}$, since $\varphi^{*}$ is increasing.

Substituting (2.12) and (2.1) into (2.11), by the subadditivity of $\omega$ we have

$$
\begin{aligned}
e^{-\lambda \varphi_{\omega}^{*}\left(\frac{|\alpha|}{\lambda}\right)-\mu \varphi_{\omega}^{*}\left(\frac{|\beta|}{\mu}\right)}\left|y^{\beta} D_{y}^{\alpha} f(y)\right| \leq & C_{\mu} \sum_{\gamma \leq \alpha}\left(\begin{array}{c}
\alpha \\
\gamma
\end{array}\right) 2^{-|\alpha|} \int_{\mathbb{R}^{2 d}}\left|V_{\varphi} f(x, \xi)\right| e^{\mu \omega(x)} e^{\mu \omega(y-x)} \\
& \cdot|\xi|^{|\alpha-\gamma|}\left|D_{y}^{\gamma} \varphi(y-x)\right| e^{-3 \lambda \varphi_{\omega}^{*}\left(\frac{|\alpha|}{3 \lambda}\right)} d x d \xi .
\end{aligned}
$$

Since $\varphi \in \mathcal{S}_{\omega}\left(\mathbb{R}^{d}\right)$, by (2.3), for every $\lambda, \mu>0$ there is a constant $C_{\lambda, \mu}>0$ such that for all $\gamma \in \mathbb{N}_{0}^{d}$ and $y \in \mathbb{R}^{d}$,

$$
\left|D_{y}^{\gamma} \varphi(y)\right| e^{\mu \omega(y)} \leq C_{\lambda, \mu} e^{\lambda \varphi_{\omega}^{*}\left(\frac{|\gamma|}{\lambda}\right)} .
$$

From (2.14) with $3 \lambda$ instead of $\lambda$ and $y-x$ instead of $y$, we have that for every $\mu, \lambda>0$ there exists a constant $C_{\mu, \lambda}>0$ such that

$$
\begin{aligned}
e^{-\lambda \varphi_{\omega}^{*}\left(\frac{|\alpha|}{\lambda}\right)-\mu \varphi_{\omega}^{*}\left(\frac{|\beta|}{\mu}\right)}\left|y^{\beta} D_{y}^{\alpha} f(y)\right| \leq & C_{\mu, \lambda} \sum_{\gamma \leq \alpha}\left(\begin{array}{l}
\alpha \\
\gamma
\end{array}\right) 2^{-|\alpha|} \\
& \cdot \int_{\mathbb{R}^{2 d}}\left|V_{\varphi} f(x, \xi)\right| e^{\mu \omega(x)}|\xi|^{|\alpha-\gamma|} e^{3 \lambda \varphi_{\omega}^{*}\left(\frac{|\gamma|}{3 \lambda}\right)-3 \lambda \varphi_{\omega}^{*}\left(\frac{|\alpha|}{3 \lambda}\right)} d x d \xi .
\end{aligned}
$$

By (2.12) we have $|\xi|^{|\alpha-\gamma|} \leq C_{3 \lambda} e^{3 \lambda \omega(\xi)+3 \lambda \varphi_{\omega}^{*}\left(\frac{|\alpha-\gamma|}{3 \lambda}\right)}$. Since $\varphi_{\omega}^{*}$ is convex and $\varphi_{\omega}^{*}(0) \leq 0$, we have that

$$
\varphi_{\omega}^{*}(a)+\varphi_{\omega}^{*}(b)=\varphi_{\omega}^{*}\left((a+b) \frac{a}{a+b}\right)+\varphi_{\omega}^{*}\left((a+b) \frac{b}{a+b}\right) \leq \varphi_{\omega}^{*}(a+b), \quad a, b>0 .
$$

Therefore, for a new constant $C_{\mu, \lambda}>0$ :

$$
\begin{aligned}
& e^{-\lambda \varphi_{\omega}^{*}\left(\frac{|\alpha|}{\lambda}\right)-\mu \varphi_{\omega}^{*}\left(\frac{|\beta|}{\mu}\right)}\left|y^{\beta} D_{y}^{\alpha} f(y)\right| \\
& \leq C_{\mu, \lambda} \sum_{\gamma \leq \alpha}\left(\begin{array}{c}
\alpha \\
\gamma
\end{array}\right) 2^{-|\alpha|} \int_{\mathbb{R}^{2 d}}\left|V_{\varphi} f(x, \xi)\right| e^{\mu \omega(x)} e^{3 \lambda \omega(\xi)} d x d \xi \\
& \leq C_{\mu, \lambda} \int_{\mathbb{R}^{2 d}}\left|V_{\varphi} f(z)\right| e^{(\mu+3 \lambda+m) \omega(z)} e^{-m \omega(z)} d z \\
& \leq C_{\mu, \lambda}^{\prime}\left\|V_{\varphi} f\right\|_{\omega, \mu+3 \lambda+m},
\end{aligned}
$$

for $C_{\mu, \lambda}^{\prime}:=C_{\mu, \lambda} \int_{\mathbb{R}^{2 d}} e^{-m \omega(z)} d z$, which is finite if $m \geq(2 d+1) / b$, where $b$ is the constant in condition $(\gamma)$ of Definition 2.1.

It is easy to see that $\tilde{\mathcal{S}}_{\omega}\left(\mathbb{R}^{d}\right)$ is a Fréchet space. Indeed, the estimate (2.16) implies that the identity operator $I: \tilde{\mathcal{S}}_{\omega}\left(\mathbb{R}^{d}\right) \rightarrow \mathcal{S}_{\omega}\left(\mathbb{R}^{d}\right)$ is continuous. Hence, any Cauchy sequence $\left\{f_{n}\right\}_{n \in \mathbb{N}}$ in $\tilde{\mathcal{S}}_{\omega}\left(\mathbb{R}^{d}\right)$ is a Cauchy sequence in $\mathcal{S}_{\omega}\left(\mathbb{R}^{d}\right)$. So, it converges in $\mathcal{S}_{\omega}\left(\mathbb{R}^{d}\right)$ to some $f$ (because $\mathcal{S}_{\omega}\left(\mathbb{R}^{d}\right)$ is complete). From Proposition $2.9,\left\{V_{\varphi} f_{n}\right\}_{n \in \mathbb{N}}$ converges to $V_{\varphi} f$ in $\mathcal{S}_{\omega}\left(\mathbb{R}^{2 d}\right)$. Therefore, $\left\{f_{n}\right\}_{n \in \mathbb{N}}$ converges to $f$ in $\tilde{\mathcal{S}}_{\omega}\left(\mathbb{R}^{d}\right)$.

We can apply the open mapping theorem to conclude that $I$ is an isomorphism and hence the two topologies on $\mathcal{S}_{\omega}\left(\mathbb{R}^{d}\right)$ coincide.

Now, we can prove the following 
Proposition 2.11. Assume that $\psi, \gamma \in \mathcal{S}_{\omega}\left(\mathbb{R}^{d}\right) \backslash\{0\}$ with $\langle\psi, \gamma\rangle \neq 0$. Then the following assertions hold:

(a) If $F: \mathbb{R}^{2 d} \rightarrow \mathbb{C}$ is a measurable function that satisfies, for some $c, \lambda>0$,

$$
|F(z)| \leq c e^{\lambda \omega(z)} \quad \forall z \in \mathbb{R}^{2 d}
$$

then

$$
\mathcal{S}_{\omega}\left(\mathbb{R}^{d}\right) \ni \varphi \mapsto\langle f, \varphi\rangle:=\int_{\mathbb{R}^{2 d}} F(z)\langle\Pi(z) \gamma, \varphi\rangle d z
$$

defines an $\omega$-tempered distribution $f \in \mathcal{S}_{\omega}^{\prime}\left(\mathbb{R}^{d}\right)$.

(b) In particular, if $F=V_{\psi} f$ for some $f \in \mathcal{S}_{\omega}^{\prime}\left(\mathbb{R}^{d}\right)$, then the following inversion formula holds:

$$
f=\frac{1}{(2 \pi)^{d}\langle\gamma, \psi\rangle} \int_{\mathbb{R}^{2 d}} V_{\psi} f(z) \Pi(z) \gamma d z
$$

Proof. From (2.17) we have, for all $\varphi \in \mathcal{S}_{\omega}\left(\mathbb{R}^{d}\right)$,

$$
\begin{aligned}
|\langle f, \varphi\rangle| & \leq \int_{\mathbb{R}^{2 d}}|F(z)| \cdot\left|V_{\gamma} \varphi(z)\right| d z \\
& \leq c \int_{\mathbb{R}^{2 d}} e^{\lambda \omega(z)+m \omega(z)}\left|V_{\gamma} \varphi(z)\right| e^{-m \omega(z)} d z \\
& \leq c^{\prime}\left\|V_{\gamma} \varphi\right\|_{\omega, \lambda+m}
\end{aligned}
$$

for some $c^{\prime}>0$ and $m \geq(2 d+1) / b$, where $b$ is the constant in condition $(\gamma)$ of Definition 2.1.

From Proposition 2.10 the inequality (2.19) implies that $f$ defines a continuous linear functional on $\mathcal{S}_{\omega}\left(\mathbb{R}^{d}\right)$, i.e. $f \in \mathcal{S}_{\omega}^{\prime}\left(\mathbb{R}^{d}\right)$. This proves $(a)$.

In particular, if $F=V_{\psi} f$ for some $f \in \mathcal{S}_{\omega}^{\prime}\left(\mathbb{R}^{d}\right)$ then $F$ satisfies $(2.17)$ by Theorem 2.5 and hence (2.18) defines an $\omega$-tempered distribution $\tilde{f} \in \mathcal{S}_{\omega}^{\prime}\left(\mathbb{R}^{d}\right)$ given by

$$
\langle\tilde{f}, \varphi\rangle=\frac{1}{(2 \pi)^{d}\langle\gamma, \psi\rangle} \int_{\mathbb{R}^{2 d}} V_{\psi} f(z)\langle\Pi(z) \gamma, \varphi\rangle d z \quad \forall \varphi \in \mathcal{S}_{\omega}\left(\mathbb{R}^{d}\right) .
$$

However, from (2.7) we have that

$$
\varphi=\frac{1}{(2 \pi)^{d}\langle\psi, \gamma\rangle} \int_{\mathbb{R}^{2 d}} V_{\gamma} \varphi(z) \Pi(z) \psi d z
$$

and then (see also [18, pg 43] for vector valued integrals)

$$
\begin{aligned}
\langle f, \varphi\rangle & =\frac{1}{(2 \pi)^{d} \overline{\langle\psi, \gamma\rangle}} \int_{\mathbb{R}^{2 d}} \overline{V_{\gamma} \varphi(z)}\langle f, \Pi(z) \psi\rangle d z \\
& =\frac{1}{(2 \pi)^{d}\langle\gamma, \psi\rangle} \int_{\mathbb{R}^{2 d}}\langle\Pi(z) \gamma, \varphi\rangle V_{\psi} f(z) d z \\
& =\langle\tilde{f}, \varphi\rangle, \quad \varphi \in \mathcal{S}_{\omega}\left(\mathbb{R}^{d}\right) .
\end{aligned}
$$

Therefore $f=\tilde{f}$ and $(b)$ is proved. 
Let us now recall the definition of the adjoint operator of $V_{\varphi}$. We consider, for $\varphi \in L^{2}\left(\mathbb{R}^{d}\right)$, the operator

$$
A_{\varphi}: L^{2}\left(\mathbb{R}^{2 d}\right) \longrightarrow L^{2}\left(\mathbb{R}^{d}\right)
$$

defined by

$$
A_{\varphi} F=\int_{\mathbb{R}^{2 d}} F(z) \Pi(z) \varphi d z
$$

This is the adjoint operator of $V_{\varphi}: L^{2}\left(\mathbb{R}^{d}\right) \rightarrow L^{2}\left(\mathbb{R}^{2 d}\right)$ since, for all $F \in L^{2}\left(\mathbb{R}^{2 d}\right)$ and $h \in L^{2}\left(\mathbb{R}^{d}\right)$,

$$
\left\langle A_{\varphi} F, h\right\rangle=\int_{\mathbb{R}^{2 d}} F(z)\langle\Pi(z) \varphi, h\rangle d z=\left\langle F, V_{\varphi} h\right\rangle=\left\langle V_{\varphi}^{*} F, h\right\rangle .
$$

In particular, for $\varphi \in \mathcal{S}_{\omega}\left(\mathbb{R}^{d}\right)$ and $F \in \mathcal{S}_{\omega}\left(\mathbb{R}^{2 d}\right)$ we can define the adjoint operator $V_{\varphi}^{*} F=$ $A_{\varphi} F$. We observe that $V_{\varphi}^{*} F \in \mathcal{S}_{\omega}\left(\mathbb{R}^{d}\right)$. In fact, if $G(x, \xi, t):=F(x, \xi) \varphi(t-x) \in \mathcal{S}_{\omega}\left(\mathbb{R}^{3 d}\right)$, we can write $A_{\varphi} F$ as a partial Fourier transform:

$$
A_{\varphi} F(t)=\int_{\mathbb{R}^{2 d}} F(x, \xi) \varphi(t-x) e^{i\langle t, \xi\rangle} d x d \xi=\left.\left(\mathcal{F}_{(x, \xi)} G\right)\left(x^{\prime}, \xi^{\prime}, t\right)\right|_{\left(x^{\prime}, \xi^{\prime}, t\right)=(0,-t, t)} .
$$

Since $\mathcal{S}_{\omega}\left(\mathbb{R}^{3 d}\right)$ is invariant under partial Fourier transforms (see, e.g. [8, Remark 4.10]) and restrictions to linear sub-manifolds we deduce that

$$
V_{\varphi}^{*}: \mathcal{S}_{\omega}\left(\mathbb{R}^{2 d}\right) \longrightarrow \mathcal{S}_{\omega}\left(\mathbb{R}^{d}\right)
$$

is continuous.

Moreover, the inversion formula $(2.7)$ gives, for $\varphi, \psi, f \in \mathcal{S}_{\omega}\left(\mathbb{R}^{d}\right)$ with $\langle\varphi, \psi\rangle \neq 0$,

$$
\frac{1}{\langle\varphi, \psi\rangle} V_{\varphi}^{*} V_{\psi} f=\frac{1}{\langle\varphi, \psi\rangle} \int_{\mathbb{R}^{2 d}} V_{\psi} f(z) \Pi(z) \varphi d z=(2 \pi)^{d} f
$$

i.e.

$$
\frac{1}{(2 \pi)^{d}\langle\varphi, \psi\rangle} V_{\varphi}^{*} V_{\psi}=I_{\mathcal{S}_{\omega}\left(\mathbb{R}^{d}\right)} .
$$

More in general, if $\varphi \in \mathcal{S}_{\omega}\left(\mathbb{R}^{d}\right) \backslash\{0\}$ and $F$ is a measurable function on $\mathbb{R}^{2 d}$, we define the adjoint operator

$$
V_{\varphi}^{*} F=\int_{\mathbb{R}^{2 d}} F(z) \Pi(z) \varphi d z
$$

where the integral is interpreted, if necessary, in a weak sense, i.e.

$$
\left\langle V_{\varphi}^{*} F, g\right\rangle=\int_{\mathbb{R}^{2 d}} F(z)\langle\Pi(z) \varphi, g\rangle d z=\int_{\mathbb{R}^{2 d}} F(z) \overline{V_{\varphi} g}(z) d z=\left\langle F, V_{\varphi} g\right\rangle
$$

for $g \in \mathcal{S}_{\omega}\left(\mathbb{R}^{d}\right)$.

In particular, if $\varphi, \psi \in \mathcal{S}_{\omega}\left(\mathbb{R}^{d}\right) \backslash\{0\}$ with $\langle\varphi, \psi\rangle \neq 0$, by Theorem 2.5 and Proposition 2.11 we can define the adjoint operator $(2.23)$ for $F=V_{\psi} f$ with $f \in \mathcal{S}_{\omega}^{\prime}\left(\mathbb{R}^{d}\right)$ and obtain that, for all $g \in \mathcal{S}_{\omega}\left(\mathbb{R}^{d}\right)$,

$$
\left\langle V_{\varphi}^{*} V_{\psi} f, g\right\rangle=\int_{\mathbb{R}^{2 d}} V_{\psi} f(z)\langle\Pi(z) \varphi, g\rangle d z=(2 \pi)^{d}\langle\varphi, \psi\rangle\langle f, g\rangle
$$


i.e.

$$
\frac{1}{(2 \pi)^{d}\langle\varphi, \psi\rangle} V_{\varphi}^{*} V_{\psi}=I_{\mathcal{S}_{\omega}^{\prime}\left(\mathbb{R}^{d}\right)}
$$

We can now prove the following proposition in a standard way.

Proposition 2.12. Let $\varphi, \psi, \gamma \in \mathcal{S}_{\omega}\left(\mathbb{R}^{d}\right)$ with $\langle\gamma, \psi\rangle \neq 0$ and let $f \in \mathcal{S}_{\omega}^{\prime}\left(\mathbb{R}^{d}\right)$. Then

$$
\left|V_{\varphi} f(z)\right| \leq \frac{1}{(2 \pi)^{d}|\langle\gamma, \psi\rangle|}\left(\left|V_{\psi} f\right| *\left|V_{\varphi} \gamma\right|\right)(z), \quad z=(x, \xi) \in \mathbb{R}^{2 d}
$$

\section{The $\omega$-Gabor wave front set}

In this section we consider a global wave front set for $\omega$-tempered distributions from two different points of view. The first one is defined in terms of rapid decay of the STFT in conical sets, that is a natural approach to analyze the regularity of an ultradistribution with respect to variables and covariables simultaneously. The second one is described in terms of the rapid decay of the Gabor frame coefficients, and is more related to applications to signal processing and related topics (see, for instance, $[35,14,20]$ ).

One of the main results of this section is to prove that these two points of view lead to the same global wave front set, so that it is actually sufficient to consider the decay of the Gabor transform in conical sets intersected with a suitable lattice.

Definition 3.1. Let $u \in \mathcal{S}_{\omega}^{\prime}\left(\mathbb{R}^{d}\right)$ and $\varphi \in \mathcal{S}_{\omega}\left(\mathbb{R}^{d}\right) \backslash\{0\}$. We say that $z_{0}=\left(x_{0}, \xi_{0}\right) \in \mathbb{R}^{2 d} \backslash\{0\}$ is not in the $\omega$-wave front set $\mathrm{WF}_{\omega}^{\prime}(u)$ of $u$ if there exists an open conic set $\Gamma \subseteq \mathbb{R}^{2 d} \backslash\{0\}$ containing $z_{0}$ and such that

$$
\sup _{z \in \Gamma} e^{\lambda \omega(z)}\left|V_{\varphi} u(z)\right|<+\infty, \quad \forall \lambda>0 .
$$

We observe that $\mathrm{WF}_{\omega}^{\prime}(u)$ is a closed conic subset of $\mathbb{R}^{2 d} \backslash\{0\}$. Moreover, it does not depend on the choice of the window function $\varphi$, as the following proposition shows.

Proposition 3.2. Let $u \in \mathcal{S}_{\omega}^{\prime}\left(\mathbb{R}^{d}\right), \varphi \in \mathcal{S}_{\omega}\left(\mathbb{R}^{d}\right) \backslash\{0\}$ and $z_{0} \in \mathbb{R}^{2 d} \backslash\{0\}$. Assume that there exists an open conic set $\Gamma \subseteq \mathbb{R}^{2 d} \backslash\{0\}$ containing $z_{0}$ such that (3.1) is satisfied. Then, for any $\psi \in \mathcal{S}_{\omega}\left(\mathbb{R}^{d}\right) \backslash\{0\}$ and for any open conic set $\Gamma^{\prime} \subseteq \mathbb{R}^{2 d} \backslash\{0\}$ containing $z_{0}$ and such that $\overline{\Gamma^{\prime} \cap S_{2 d-1}} \subseteq \Gamma$, where $S_{2 d-1}$ is the unit sphere in $\mathbb{R}^{2 d}$, we have

$$
\sup _{z \in \Gamma^{\prime}} e^{\lambda \omega(z)}\left|V_{\psi} u(z)\right|<+\infty, \quad \forall \lambda>0 .
$$

Proof. From Proposition 2.12 we have that

$$
\left|V_{\psi} u(z)\right| \leq(2 \pi)^{-d}\|\varphi\|_{L^{2}}^{-2}\left(\left|V_{\varphi} u\right| *\left|V_{\psi} \varphi\right|\right)(z) \quad \forall z \in \mathbb{R}^{2 d} .
$$

Moreover, since $\varphi \in \mathcal{S}_{\omega}\left(\mathbb{R}^{d}\right)$, from Theorem 2.7 we have that for every $\mu>0$ there exists $C_{\mu}>0$ such that

$$
e^{\mu \omega(z)}\left|V_{\psi} \varphi(z)\right| \leq C_{\mu} \quad \forall z \in \mathbb{R}^{2 d} .
$$


Then

$$
\begin{aligned}
\left(\left|V_{\varphi} u\right| *\left|V_{\psi} \varphi\right|\right)(z) & =\int_{\mathbb{R}^{2 d}}\left|V_{\varphi} u\left(z-z^{\prime}\right)\right| \cdot\left|V_{\psi} \varphi\left(z^{\prime}\right)\right| d z^{\prime} \\
& =\int_{\left\langle z^{\prime}\right\rangle \leq \varepsilon\langle z\rangle}\left|V_{\varphi} u\left(z-z^{\prime}\right)\right| \cdot\left|V_{\psi} \varphi\left(z^{\prime}\right)\right| d z^{\prime}+\int_{\left\langle z^{\prime}\right\rangle>\varepsilon\langle z\rangle}\left|V_{\varphi} u\left(z-z^{\prime}\right)\right| \cdot\left|V_{\psi} \varphi\left(z^{\prime}\right)\right| d z^{\prime} \\
& =: I_{1}+I_{2} .
\end{aligned}
$$

Let us choose $\varepsilon>0$ sufficiently small so that

$$
z \in \Gamma^{\prime},|z| \geq 1,\left\langle z^{\prime}\right\rangle \leq \varepsilon\langle z\rangle \quad \Rightarrow \quad z-z^{\prime} \in \Gamma,
$$

and hence, from (3.1), the subadditivity of $\omega$ and (3.4):

$$
\begin{aligned}
I_{1} & \leq C_{\lambda} \int_{\left\langle z^{\prime}\right\rangle \leq \varepsilon(z\rangle} e^{-\lambda \omega\left(z-z^{\prime}\right)}\left|V_{\psi} \varphi\left(z^{\prime}\right)\right| d z^{\prime} \\
& \leq C_{\lambda} e^{-\lambda \omega(z)} \int_{\mathbb{R}^{2 d}} e^{(\lambda+m) \omega\left(z^{\prime}\right)}\left|V_{\psi} \varphi\left(z^{\prime}\right)\right| e^{-m \omega\left(z^{\prime}\right)} d z^{\prime} \\
& \leq C_{\lambda}^{\prime} e^{-\lambda \omega(z)}, \quad \lambda>0, \quad z \in \Gamma^{\prime},|z| \geq 1 .
\end{aligned}
$$

if $m \geq(2 d+1) / b$, where $b$ is the constant in condition $(\gamma)$ of Definition 2.1.

On the other hand, from Theorem 2.5 and (3.4), for $m>0$ big enough:

$$
\begin{aligned}
I_{2} & \leq c \int_{\left\langle z^{\prime}\right\rangle>\varepsilon\langle z\rangle} e^{\lambda \omega\left(z-z^{\prime}\right)}\left|V_{\psi} \varphi\left(z^{\prime}\right)\right| d z^{\prime} \\
& \leq c e^{\lambda \omega(z)} \int_{\left\langle z^{\prime}\right\rangle>\varepsilon\langle z\rangle} e^{(\lambda+m-\mu) \omega\left(z^{\prime}\right)}\left|V_{\psi} \varphi\left(z^{\prime}\right)\right| e^{\mu \omega\left(z^{\prime}\right)} e^{-m \omega\left(z^{\prime}\right)} d z^{\prime} \\
& \leq c^{\prime} e^{\lambda \omega(z)} e^{-A(\lambda+m-\mu)} e^{(\lambda+m-\mu) B_{\varepsilon} \omega(z)} C_{\mu}
\end{aligned}
$$

for some $c^{\prime}>0$, if $\mu>\lambda+m, A=\omega(1)$ and $B_{\varepsilon}=([1 / \varepsilon]+1)^{-1}$, since for $\left\langle z^{\prime}\right\rangle>\varepsilon\langle z\rangle$ by the subadditivity of $\omega$ :

$$
\begin{aligned}
\omega(z) & \leq \omega(\langle z\rangle) \leq \omega\left(\frac{1}{\varepsilon}\left\langle z^{\prime}\right\rangle\right) \leq\left(\left[\frac{1}{\varepsilon}\right]+1\right) \omega\left(\left\langle z^{\prime}\right\rangle\right) \\
& \leq\left(\left[\frac{1}{\varepsilon}\right]+1\right) \omega\left(1+\left|z^{\prime}\right|\right) \leq\left(\left[\frac{1}{\varepsilon}\right]+1\right)\left(\omega(1)+\omega\left(z^{\prime}\right)\right),
\end{aligned}
$$

where $[x]$ denotes the integer part of $x \in \mathbb{R}$.

Since $\varepsilon$ is fixed, the arbitrariness of $\mu>\lambda+m$ in (3.7) implies that for every $\lambda^{\prime}>0$ there exists a constant $C_{\lambda^{\prime}}>0$ such that

$$
I_{2} \leq C_{\lambda^{\prime}} e^{-\lambda^{\prime} \omega(z)}, \quad z \in \mathbb{R}^{2 d} .
$$

This gives the conclusion.

Given $\alpha, \beta>0$, consider the lattice $\Lambda=\alpha \mathbb{Z}^{d} \times \beta \mathbb{Z}^{d} \subset \mathbb{R}^{2 d}$. For a window $\varphi \in L^{2}\left(\mathbb{R}^{d}\right) \backslash\{0\}$ the collection $\{\Pi(\sigma) \varphi\}_{\sigma \in \Lambda}$ is called a Gabor frame for $L^{2}\left(\mathbb{R}^{d}\right)$ provided there exist constants $A, B>0$ such that

$$
A\|f\|_{L^{2}}^{2} \leq \sum_{\sigma \in \Lambda}|\langle f, \Pi(\sigma) \varphi\rangle|^{2} \leq B\|f\|_{L^{2}}^{2}, \quad f \in L^{2}\left(\mathbb{R}^{d}\right)
$$


(see [18] for the analysis of the conditions on $\alpha$ and $\beta$ for which $\{\Pi(\sigma) \varphi\}_{\sigma \in \Lambda}$ is a Gabor frame). Now, we define the Gabor $\omega$-wave front set.

Definition 3.3. Let $\varphi \in \mathcal{S}_{\omega}\left(\mathbb{R}^{d}\right) \backslash\{0\}$ and $\Lambda=\alpha_{0} \mathbb{Z}^{d} \times \beta_{0} \mathbb{Z}^{d} \subseteq \mathbb{R}^{2 d}$ a lattice with $\alpha_{0}, \beta_{0}>0$ sufficiently small so that $\{\Pi(\sigma) \varphi\}_{\sigma \in \Lambda}$ is a Gabor frame for $L^{2}\left(\mathbb{R}^{d}\right)$. If $u \in \mathcal{S}_{\omega}^{\prime}\left(\mathbb{R}^{d}\right)$, we say that $z_{0} \in \mathbb{R}^{2 d} \backslash\{0\}$ is not in the Gabor $\omega$-wave front set $\mathrm{WF}_{\omega}^{G}(u)$ of $u$ if there exists an open conic set $\Gamma \subset \mathbb{R}^{2 d} \backslash\{0\}$ containing $z_{0}$ such that

$$
\sup _{\sigma \in \Lambda \cap \Gamma} e^{\lambda \omega(\sigma)}\left|V_{\varphi} u(\sigma)\right|<+\infty \quad \forall \lambda>0 .
$$

Our next goal is to prove that $\mathrm{WF}_{\omega}^{\prime}(u)=\mathrm{WF}_{\omega}^{G}(u)$. To this aim we need some properties of modulation spaces adapted to our setting. We prove those results that differ from the classical ones already known in $\mathcal{S}\left(\mathbb{R}^{d}\right)$ (see $\left.[18]\right)$.

We consider, for $\lambda \in \mathbb{R} \backslash\{0\}$,

$$
m_{\lambda}(z)=e^{\lambda \omega(z)}, \quad v_{\lambda}(z)=e^{|\lambda| \omega(z)}, \quad z \in \mathbb{R}^{n} .
$$

The weights $m_{\lambda}(z)$ are $v_{\lambda}$-moderate, in the sense that

$$
m_{\lambda}\left(z_{1}+z_{2}\right) \leq v_{\lambda}\left(z_{1}\right) m_{\lambda}\left(z_{2}\right)
$$

for every $\lambda \neq 0$ and $z_{1}, z_{2} \in \mathbb{R}^{n}$. This is immediate from the subadditivity of $\omega$.

We denote, following [18], the weighted $L^{p, q}$ spaces by

$$
\begin{aligned}
L_{m_{\lambda}}^{p, q}\left(\mathbb{R}^{2 d}\right):=\left\{F \text { measurable on } \mathbb{R}^{2 d}\right. \text { such that } & \\
& \left.\|F\|_{L_{m_{\lambda}}^{p, q}}:=\left(\int_{\mathbb{R}^{d}}\left(\int_{\mathbb{R}^{d}}|F(x, \xi)|^{p} m_{\lambda}(x, \xi)^{p} d x\right)^{q / p} d \xi\right)^{1 / q}<+\infty\right\},
\end{aligned}
$$

for $1 \leq p, q<+\infty$, and

$$
\begin{aligned}
& L_{m_{\lambda}}^{\infty, q}\left(\mathbb{R}^{2 d}\right):=\left\{F \text { measurable on } \mathbb{R}^{2 d}\right. \text { such that } \\
&\left.\|F\|_{L_{m_{\lambda}}^{\infty, q}}:=\left(\int_{\mathbb{R}^{d}}\left(\underset{x \in \mathbb{R}^{d}}{\operatorname{ess} \sup }|F(x, \xi)| m_{\lambda}(x, \xi)\right)^{q} d \xi\right)^{1 / q}<+\infty\right\}, \\
& L_{m_{\lambda}}^{p, \infty}\left(\mathbb{R}^{2 d}\right):=\left\{F \text { measurable on } \mathbb{R}^{2 d}\right. \text { such that } \\
&\left.\|F\|_{L_{m_{\lambda}}^{p, \infty}}:=\operatorname{ess~sup}_{\xi \in \mathbb{R}^{d}}\left(\int_{\mathbb{R}^{d}}|F(x, \xi)|^{p} m_{\lambda}(x, \xi)^{p} d x\right)^{1 / p}<+\infty\right\},
\end{aligned}
$$

for $1 \leq p, q \leq+\infty$ with $p=+\infty$ or $q=+\infty$ respectively.

By [18, Lemma 11.1.2] these are Banach spaces for all $1 \leq p, q \leq+\infty$. Moreover, for $F \in L_{m_{\lambda}}^{p, q}\left(\mathbb{R}^{2 d}\right)$ and $H \in L_{1 / m_{\lambda}}^{p^{\prime}, q^{\prime}}\left(\mathbb{R}^{2 d}\right)$, where $p^{\prime}$ and $q^{\prime}$ are the conjugate exponents of $p$ and $q$ respectively (i.e. $\frac{1}{p}+\frac{1}{p^{\prime}}=1$ if $1<p<+\infty, p^{\prime}=+\infty$ if $p=1, p^{\prime}=1$ if $p=+\infty$, and the same for $q)$, then $F \cdot H \in L^{1}\left(\mathbb{R}^{2 d}\right)$ and

$$
\left|\int_{\mathbb{R}^{2 d}} F(z) \overline{H(z)} d z\right| \leq\|F\|_{L_{m_{\lambda}}^{p, q}}\|H\|_{L_{1 / m_{\lambda}}^{p^{\prime}, q^{\prime}}} .
$$

If $1 \leq p, q<+\infty$, the dual of $L_{m_{\lambda}}^{p, q}\left(\mathbb{R}^{2 d}\right)$ is given by $L_{1 / m_{\lambda}}^{p^{\prime}, q^{\prime}}\left(\mathbb{R}^{2 d}\right)$. 
From [18, Proposition 11.1.3] we have the following Young inequality for weighted $L^{p, q}$ spaces. For $F \in L_{m_{\lambda}}^{p, q}$ and $G \in L_{v_{\lambda}}^{1}$,

$$
\|F * G\|_{L_{m_{\lambda}}^{p, q}} \leq C\|F\|_{L_{m_{\lambda}}^{p, q}}\|G\|_{L_{v_{\lambda}}^{1}}
$$

for some $C>0$.

Remark 3.4. It is easy to see that for every $\lambda \in \mathbb{R} \backslash\{0\}$ and $1 \leq p, q \leq+\infty$ we have

$$
\mathcal{S}_{\omega}\left(\mathbb{R}^{2 d}\right) \subset L_{m_{\lambda}}^{p, q}\left(\mathbb{R}^{2 d}\right) .
$$

Moreover $e^{-\mu \omega(z)} \in L_{m_{\lambda}}^{p, q}\left(\mathbb{R}^{2 d}\right)$ for $\mu>0$ large enough, since $m_{\lambda}(z)=e^{\lambda \omega(z)}$ and $e^{-A \omega(z)} \leq$ $e^{-a A}(1+|z|)^{-b A}$ for any $A>0$, by condition $(\gamma)$ of Definition 2.1.

Definition 3.5. Let $\varphi \in \mathcal{S}_{\omega}\left(\mathbb{R}^{d}\right) \backslash\{0\}$, and $m_{\lambda}(z)$ as in (3.10) for some $\lambda \neq 0$. For $1 \leq p, q \leq$ $+\infty$, the modulation space $\boldsymbol{M}_{m_{\lambda}}^{p, q}\left(\mathbb{R}^{d}\right)$ is defined by

$$
\boldsymbol{M}_{m_{\lambda}}^{p, q}\left(\mathbb{R}^{d}\right):=\left\{f \in \mathcal{S}_{\omega}^{\prime}\left(\mathbb{R}^{d}\right): V_{\varphi} f \in L_{m_{\lambda}}^{p, q}\left(\mathbb{R}^{2 d}\right)\right\},
$$

with norm $\|f\|_{M_{m_{\lambda}}^{p, q}}=\left\|V_{\varphi} f\right\|_{L_{m_{\lambda}}^{p, q}}$. We denote then $\boldsymbol{M}_{m_{\lambda}}^{p}\left(\mathbb{R}^{d}\right):=\boldsymbol{M}_{m_{\lambda}}^{p, p}\left(\mathbb{R}^{d}\right)$.

Observe that Definition 3.5 is similar to the definition of modulation spaces in [18]; the difference is that here $\boldsymbol{M}_{m_{\lambda}}^{p, q}\left(\mathbb{R}^{d}\right)$ is a subset of $\mathcal{S}_{\omega}^{\prime}\left(\mathbb{R}^{d}\right)$, and we take a window $\varphi \in \mathcal{S}_{\omega}\left(\mathbb{R}^{d}\right)$, while in [18] the modulation space $M_{m}^{p, q}\left(\mathbb{R}^{d}\right)$ is a subset of $\mathcal{S}^{\prime}\left(\mathbb{R}^{d}\right)$ and the window belongs to $\mathcal{S}\left(\mathbb{R}^{d}\right)$ (or a subset of $\left(M_{v}^{1}\right)^{*}$ for a suitable weight $v$, in a suitable space of 'special' windows $\mathcal{S}_{\mathcal{C}}\left(\mathbb{R}^{d}\right)$ ). Moreover, here we always need weights of exponential type. We refer to $[40,41]$ for modulation spaces in the setting of Gelfand-Shilov spaces, among other type of spaces of ultradifferentiable functions and ultradistributions.

The definition of $\boldsymbol{M}_{m_{\lambda}}^{p, q}$ is independent of the window $\varphi$, in the sense that different (non-zero) windows in $\mathcal{S}_{\omega}\left(\mathbb{R}^{d}\right)$ give equivalent norms. Indeed for $\varphi, \psi \in \mathcal{S}_{\omega}\left(\mathbb{R}^{d}\right), \varphi, \psi \neq 0$, we have from Proposition 2.12, applied with $\gamma=\psi$, that

$$
\left\|V_{\varphi} f\right\|_{L_{m_{\lambda}}^{p, q}} \leq \frac{1}{(2 \pi)^{d}\|\psi\|_{L^{2}}^{2}}\left\|\left|V_{\psi} f\right| *\left|V_{\varphi} \psi\right|\right\|_{L_{m_{\lambda}}^{p, q}} \leq C\left\|V_{\psi} f\right\|_{L_{m_{\lambda}}^{p, q},}
$$

where $C=\frac{\left\|V_{\varphi} \psi\right\|_{L_{\lambda}^{1}}^{1}}{(2 \pi)^{d}\|\psi\|_{L^{2}}^{2}}$, as we can deduce from Young inequality (3.12) (observe that $C$ is finite by Proposition 2.9 and Remark 3.4). Then, by interchanging the roles of $\varphi$ and $\psi$ we have that $V_{\varphi} f \in L_{m_{\lambda}}^{p, q}$ if and only if $V_{\psi} f \in L_{m_{\lambda}}^{p, q}$, and the corresponding modulation space norms of $f$ with respect to the two windows are equivalent.

Remark 3.6. From Theorems 2.7 and 2.5 and Proposition 2.11 we have that

$$
\mathcal{S}_{\omega}\left(\mathbb{R}^{d}\right)=\bigcap_{\lambda>0} \boldsymbol{M}_{m_{\lambda}}^{\infty}\left(\mathbb{R}^{d}\right) ; \quad \mathcal{S}_{\omega}^{\prime}\left(\mathbb{R}^{d}\right)=\bigcup_{\lambda<0} \boldsymbol{M}_{m_{\lambda}}^{\infty}\left(\mathbb{R}^{d}\right)
$$

The inversion formula of Proposition 2.11 holds also in modulation spaces, as stated below.

Proposition 3.7. Let $\gamma \in \mathcal{S}_{\omega}\left(\mathbb{R}^{d}\right)$ be a not identically zero window, and consider, for a measurable function $F$ on $\mathbb{R}^{2 d}$, the adjoint $V_{\gamma}^{*} F$ defined as in (2.23). Then: 
(i) The operator $V_{\gamma}^{*}$ acts continuously as

$$
V_{\gamma}^{*}: L_{m_{\lambda}}^{p, q}\left(\mathbb{R}^{2 d}\right) \rightarrow M_{m_{\lambda}}^{p, q}\left(\mathbb{R}^{d}\right)
$$

and there exists $C>0$ such that

$$
\left\|V_{\gamma}^{*} F\right\|_{M_{m_{\lambda}}^{p, q}} \leq C\left\|V_{\varphi} \gamma\right\|_{L_{v_{\lambda}}^{1}}\|F\|_{L_{m_{\lambda}}^{p, q}}
$$

where $\varphi$ is the window in the corresponding $\boldsymbol{M}_{m_{\lambda}}^{p, q}$ norm.

(ii) In the particular case when $F=V_{g} f$, for $g \in \mathcal{S}_{\omega}\left(\mathbb{R}^{d}\right)$, and $f \in \boldsymbol{M}_{m_{\lambda}}^{p, q}$, if $\langle\gamma, g\rangle \neq 0$ the following inversion formula holds:

$$
f=\frac{1}{(2 \pi)^{d}\langle\gamma, g\rangle} \int_{\mathbb{R}^{2 d}} V_{g} f(z) \Pi(z) \gamma d z
$$

Proof. (i) We start by proving that $V_{\gamma}^{*} F$ is an element of $\mathcal{S}_{\omega}^{\prime}\left(\mathbb{R}^{d}\right)$. For $\psi \in \mathcal{S}_{\omega}\left(\mathbb{R}^{d}\right)$ we have from (3.11),

$$
\begin{aligned}
\left|\left\langle V_{\gamma}^{*} F, \psi\right\rangle\right| & =\left|\left\langle F, V_{\gamma} \psi\right\rangle\right| \leq\|F\|_{L_{m_{\lambda}}^{p, q}\left\|V_{\gamma} \psi\right\|_{L_{1 / m_{\lambda}}^{p^{\prime}, q^{\prime}}}} \\
& \leq\|F\|_{L_{m_{\lambda}}^{p, q}}\left\|e^{\mu \omega(z)} V_{\gamma} \psi\right\|_{\infty}\left\|e^{-\mu \omega(z)}\right\|_{L_{1 / m_{\lambda}}^{p^{\prime}, q^{\prime}}} ;
\end{aligned}
$$

this expression is finite for $\mu>0$ sufficiently large, as we can deduce from Theorem 2.7(ii) and Remark 3.4. Then from Proposition 2.10 we have that $V_{\gamma}^{*} F$ is a well defined element of $\mathcal{S}_{\omega}^{\prime}\left(\mathbb{R}^{2 d}\right)$. From Theorem 2.5 we have that $V_{\varphi} V_{\gamma}^{*} F$ is a continuous function; it is explicitly given by

$$
V_{\varphi} V_{\gamma}^{*} F(z)=\left\langle V_{\gamma}^{*} F, \Pi(z) \varphi\right\rangle=\int_{\mathbb{R}^{2 d}} F(y, \eta) \overline{V_{\gamma}(\Pi(z) \varphi)(y, \eta)} d y d \eta .
$$

Writing $z=(x, \xi)$ we have

$$
\begin{aligned}
\left|V_{\varphi} V_{\gamma}^{*} F(x, \xi)\right| & =\left|\int_{\mathbb{R}^{2 d}} F(y, \eta) V_{\varphi} \gamma(x-y, \xi-\eta) e^{-i\langle y, \xi-\eta\rangle} d y d \eta\right| \\
& \leq\left(|F| *\left|V_{\varphi} \gamma\right|\right)(x, \xi) .
\end{aligned}
$$

Then, from Young inequality (3.12) we obtain

$$
\left\|V_{\gamma}^{*} F\right\|_{M_{m_{\lambda}}^{p, q}}=\left\|V_{\varphi} V_{\gamma}^{*} F\right\|_{L_{m_{\lambda}}^{p, q}} \leq C\|F\|_{L_{m_{\lambda}}^{p, q}}\left\|V_{\varphi} \gamma\right\|_{L_{v_{\lambda}}^{1}},
$$

and this expression is finite since $V_{\varphi} \gamma \in \mathcal{S}_{\omega}\left(\mathbb{R}^{2 d}\right) \subset L_{v_{\lambda}}^{1}\left(\mathbb{R}^{2 d}\right)$ for every $\lambda \in \mathbb{R}$ from Remark 3.4.

(ii) We first observe that, by (3.13), $V_{g} f \in L_{m_{\lambda}}^{p, q}$. Then, from point (i), $\tilde{f}=\frac{1}{(2 \pi)^{d}\langle\gamma, g\rangle} V_{\gamma}^{*} V_{g} f \in$ $\boldsymbol{M}_{m_{\lambda}}^{p, q}$. Since $\boldsymbol{M}_{m_{\lambda}}^{p, q} \subset \mathcal{S}_{\omega}^{\prime}$, we have that $\tilde{f}=f$ by $(2.25)$.

Theorem 3.8. Let $1 \leq p, q<\infty$. We have

$$
\left(\boldsymbol{M}_{m_{\lambda}}^{p, q}\right)^{*}=\boldsymbol{M}_{1 / m_{\lambda}}^{p^{\prime}, q^{\prime}}
$$

and the duality is given by

$$
\langle f, h\rangle=\int_{\mathbb{R}^{2 d}} V_{\varphi} f(z) \overline{V_{\varphi} h(z)} d z
$$

for $f \in M_{m_{\lambda}}^{p, q}$ and $h \in M_{1 / m_{\lambda}}^{p^{\prime}, q^{\prime}}$. 
Proof. The proof of this result relies on the duality of weighted $L^{p, q}$ spaces, and it is the same as in Theorem 11.3.6 of [18].

Proposition 3.9. For $1 \leq p, q<\infty$ we have that $\mathcal{S}_{\omega}\left(\mathbb{R}^{d}\right)$ is a dense subspace of $\boldsymbol{M}_{m_{\lambda}}^{p, q}$.

Proof. We first observe that, from property $(\gamma)$ of the weight function $\omega$ (see Definition 2.1) we have that, for $\mu>\lambda$ big enough, $e^{-\mu \omega(z)} \in L_{m_{\lambda}}^{p, q}$ by Remark 3.4. Hence, for every $f \in \mathcal{S}_{\omega}\left(\mathbb{R}^{d}\right)$ we obtain

$$
\|f\|_{M_{m_{\lambda}}^{p, q}}=\left\|V_{\varphi} f\right\|_{L_{m_{\lambda}}^{p, q}} \leq\left\|V_{\varphi} f(z) e^{\mu \omega(z)}\right\|_{\infty}\left\|e^{-\mu \omega(z)}\right\|_{L_{m_{\lambda}}^{p, q}}
$$

From Proposition 2.10 we have

$$
\mathcal{S}_{\omega}\left(\mathbb{R}^{d}\right) \subset \boldsymbol{M}_{m_{\lambda}}^{p, q}
$$

with continuous inclusion. It remains to prove the density. We denote by $K_{n}:=\left\{z \in \mathbb{R}^{2 d}\right.$ : $|z| \leq n\}$, and we fix $\varphi \in \mathcal{S}_{\omega}$ with $\|\varphi\|_{L^{2}}^{2}=(2 \pi)^{-d}$. Consider $f \in \boldsymbol{M}_{m_{\lambda}}^{p, q}$ and define

$$
F_{n}=V_{\varphi} f \cdot \chi_{K_{n}} \text { and } f_{n}=V_{\varphi}^{*} F_{n} .
$$

From Proposition 2.6 we have that $f_{n} \in \mathcal{S}_{\omega}\left(\mathbb{R}^{d}\right)$. Moreover, using (2.25) and Proposition 3.7 we obtain

$$
\begin{aligned}
\left\|f_{n}-f\right\|_{M_{m_{\lambda}}^{p, q}} & =\left\|V_{\varphi}^{*} F_{n}-V_{\varphi}^{*} V_{\varphi} f\right\|_{M_{m_{\lambda}}^{p, q}} \\
& \leq C\left\|F_{n}-V_{\varphi} f\right\|_{L_{m_{\lambda}}^{p, q}} \\
& =C\left\|V_{\varphi} f\right\|_{L_{m_{\lambda}}^{p, q}\left(\mathbb{R}^{2 d} \backslash K_{n}\right)} .
\end{aligned}
$$

So, $\left\|f_{n}-f\right\|_{\boldsymbol{M}_{m_{\lambda}}^{p, q}}$ tends to 0 for $n \rightarrow \infty$, which completes the proof.

We recall now from [18] some basic facts about amalgam spaces.

Definition 3.10. We indicate with $\ell_{m_{\lambda}}^{p, q}\left(\mathbb{Z}^{2 d}\right)$ the space of all sequences $\left(a_{k n}\right)_{k, n \in \mathbb{Z}^{d}}$, with $a_{k n} \in \mathbb{C}$ for every $k, n \in \mathbb{Z}^{d}$, such that the following norm is finite

$$
\|a\|_{\ell_{m_{\lambda}}^{p, q}}=\left(\sum_{n \in \mathbb{Z}^{d}}\left(\sum_{k \in \mathbb{Z}^{d}}\left|a_{k n}\right|^{p} m_{\lambda}(k, n)^{p}\right)^{q / p}\right)^{1 / q} .
$$

Definition 3.11. Let $F$ be a measurable function on $\mathbb{R}^{2 d}$, and define

$$
a_{k n}=\operatorname{ess~sup}_{(x, \xi) \in[0,1]^{2 d}}|F(k+x, n+\xi)| .
$$

We say that $F \in W\left(L_{m_{\lambda}}^{p, q}\right)$ if the sequence $a=\left(a_{k n}\right)_{k, n \in \mathbb{Z}^{d}}$ belongs to $\ell_{m_{\lambda}}^{p, q}\left(\mathbb{Z}^{2 d}\right)$. The space $W\left(L_{m_{\lambda}}^{p, q}\right)$ is called amalgam space, and has the norm defined by

$$
\|F\|_{W\left(L_{m_{\lambda}}^{p, q}\right)}=\|a\|_{\ell_{m_{\lambda}}^{p, q}}
$$

Let $\varphi \in \mathcal{S}_{\omega}\left(\mathbb{R}^{d}\right)$ and $\Lambda=\alpha_{0} \mathbb{Z}^{d} \times \beta_{0} \mathbb{Z}^{d}$ a lattice with $\alpha_{0}, \beta_{0}>0$ sufficiently small so that $\{\Pi(\sigma) \varphi\}_{\sigma \in \Lambda}$ is a Gabor frame for $L^{2}\left(\mathbb{R}^{d}\right)$. We indicate with $\widetilde{m}_{\lambda}$ the restriction of the weight (3.10) to the lattice $\Lambda$, in the sense that

$$
\widetilde{m}_{\lambda}(k, n):=m_{\lambda}\left(\alpha_{0} k, \beta_{0} n\right) .
$$

We recall the following result (see Proposition 11.1.4 of [18]). 
Proposition 3.12. Let $F \in W\left(L_{m_{\lambda}}^{p, q}\right)$ be a continuous function, and $\alpha_{0}, \beta_{0}>0$. Then $\left.F\right|_{\Lambda} \in$ $\ell_{\widetilde{m}_{\lambda}}^{p, q}$, and there exists a constant $C=C\left(\alpha_{0}, \beta_{0}, \lambda\right)$ such that

$$
\left\|\left.F\right|_{\Lambda}\right\|_{\ell_{\tilde{m}_{\lambda}}^{p, q}} \leq C\|F\|_{W\left(L_{m_{\lambda}}^{p, q}\right)}
$$

Now, we study the Gabor frame operator associated to the lattice $\Lambda$, given by

$$
S_{\varphi, \psi} f=\sum_{\sigma \in \Lambda}\langle f, \Pi(\sigma) \varphi\rangle \Pi(\sigma) \psi,
$$

for $\varphi, \psi, f \in L^{2}\left(\mathbb{R}^{d}\right)$.

We write as usual $S_{\varphi, \psi}=D_{\psi} C_{\varphi}$, where $C_{\varphi}$ is the 'analysis' operator, acting on a function $f$ as

$$
C_{\varphi} f=\langle f, \Pi(\sigma) \varphi\rangle, \quad \sigma \in \Lambda,
$$

and $D_{\psi}$ is the 'synthesis' operator, acting on a sequence $c=\left(c_{k n}\right)_{k, n \in \mathbb{Z}^{d}}$ as

$$
D_{\psi} c=\sum_{k, n \in \mathbb{Z}^{d}} c_{k n} \Pi\left(\alpha_{0} k, \beta_{0} n\right) \psi .
$$

We analyze the action of the previous operators on the modulation spaces $\boldsymbol{M}_{m_{\lambda}}^{p, q}$. The proofs of the next two results are very similar to [18, Thms. 12.2.3, 12.2.4], so we omit them. We just remark that, since $\varphi \in \mathcal{S}_{\omega} \subset \mathcal{S}$, we have that $V_{\varphi} \varphi \in \mathcal{S}$; then by Proposition 12.1.11 of [18] we have $V_{\varphi} \varphi \in W\left(L_{v_{\lambda}}^{1}\right)$, and so we can apply Theorem 11.1.5 of [18].

Theorem 3.13. Let $\varphi \in \mathcal{S}_{\omega}\left(\mathbb{R}^{d}\right)$ and $\Lambda$ a lattice as before. Then the operator

$$
C_{\varphi}: M_{m_{\lambda}}^{p, q}\left(\mathbb{R}^{d}\right) \longrightarrow \ell_{\widetilde{m}_{\lambda}}^{p, q}\left(\mathbb{Z}^{2 d}\right)
$$

is bounded for every $\lambda \in \mathbb{R} \backslash\{0\}, \alpha_{0}, \beta_{0}>0$, and $1 \leq p, q \leq \infty$.

Theorem 3.14. Let $\psi \in \mathcal{S}_{\omega}\left(\mathbb{R}^{d}\right)$. Then we have:

(i) The operator

$$
D_{\psi}: \ell_{\tilde{m}_{\lambda}}^{p, q}\left(\mathbb{Z}^{2 d}\right) \longrightarrow M_{m_{\lambda}}^{p, q}\left(\mathbb{R}^{d}\right)
$$

is bounded, for every $1 \leq p, q \leq \infty, \alpha_{0}, \beta_{0}>0$, and $\lambda \in \mathbb{R} \backslash\{0\}$.

(ii) For every $c \in \ell_{\widetilde{m}_{-\lambda}}^{p^{\prime}, q^{\prime}}$ and $f \in \boldsymbol{M}_{m_{\lambda}}^{p, q}$ we have that

$$
\left\langle D_{\psi} c, f\right\rangle=\left\langle c, C_{\psi} f\right\rangle, \quad \text { for } 1 \leq p, q<\infty
$$

and

$$
\left\langle C_{\psi} f, c\right\rangle=\left\langle f, D_{\psi} c\right\rangle, \quad \text { for } 1<p, q \leq \infty .
$$

(iii) For $p, q<\infty$, we have that $D_{\psi} c$ converges unconditionally in $\boldsymbol{M}_{m_{\lambda}}^{p, q}$; if $p=q=\infty$, then $D_{\psi}$ c converges unconditionally weak ${ }^{*}$ in $\boldsymbol{M}_{1 / v_{\lambda}}^{\infty}$.

Now, we study the Gabor frame operator (3.15). We recall (see [18, Prop. 5.1.1 and 5.2.1]) that if we take a window $\varphi \in L^{2}\left(\mathbb{R}^{d}\right)$ and a lattice $\Lambda$ such that $\{\Pi(\sigma) \varphi\}_{\sigma \in \Lambda}$ is a Gabor frame for $L^{2}\left(\mathbb{R}^{d}\right)$, the operator $(3.15)$ is invertible on $L^{2}\left(\mathbb{R}^{d}\right)$. Moreover, if we define the dual window $\psi$ of $\varphi$ by $\psi:=S_{\varphi, \varphi}^{-1} \varphi$, we have that for every $f \in L^{2}\left(\mathbb{R}^{d}\right)$,

$$
f=\sum_{\sigma \in \Lambda}\langle f, \Pi(\sigma) \varphi\rangle \Pi(\sigma) \psi
$$


with unconditional convergence in $L^{2}\left(\mathbb{R}^{d}\right)$. We observe also that if $\varphi \in \mathcal{S}_{\omega}\left(\mathbb{R}^{d}\right)$ then the dual window $\psi \in \mathcal{S}_{\omega}\left(\mathbb{R}^{d}\right)$ by $[19$, Thm. 4.2].

Lemma 3.15. Fix $\varphi \in \mathcal{S}_{\omega}\left(\mathbb{R}^{d}\right) \backslash\{0\}$, and let $\psi \in \mathcal{S}_{\omega}\left(\mathbb{R}^{d}\right) \backslash\{0\}$ be the dual window of $\varphi$. For $f \in \boldsymbol{M}_{m_{\lambda}}^{p, q}\left(\mathbb{R}^{d}\right), \lambda \in \mathbb{R} \backslash\{0\}$, we have

$$
f=D_{\psi} C_{\varphi} f=\sum_{\sigma \in \Lambda}\langle f, \Pi(\sigma) \varphi\rangle \Pi(\sigma) \psi
$$

and

$$
f=D_{\varphi} C_{\psi} f=\sum_{\sigma \in \Lambda}\langle f, \Pi(\sigma) \psi\rangle \Pi(\sigma) \varphi
$$

with convergence in $\boldsymbol{M}_{m_{\lambda}}^{p, q}$ for $p, q<\infty$, and weak* convergence in $\boldsymbol{M}_{1 / v_{\lambda}}^{\infty}$ in the case $p=q=\infty$.

Proof. We first consider the case $p, q<\infty$. From Proposition 3.9 we have that there exists a sequence $f_{n} \in \mathcal{S}_{\omega}\left(\mathbb{R}^{d}\right)$ such that $f_{n} \rightarrow f$ in $M_{m_{\lambda}}^{p, q}$ as $n \rightarrow \infty$. Since $\mathcal{S}_{\omega}\left(\mathbb{R}^{d}\right) \subset L^{2}\left(\mathbb{R}^{d}\right)$, we have that

$$
f_{n}=D_{\psi} C_{\varphi} f_{n}=D_{\varphi} C_{\psi} f_{n} .
$$

From Theorems 3.13 and 3.14 we obtain $D_{\psi} C_{\varphi} f_{n} \rightarrow D_{\psi} C_{\varphi} f$ and $D_{\varphi} C_{\psi} f_{n} \rightarrow D_{\varphi} C_{\psi} f$ in $\boldsymbol{M}_{m_{\lambda}}^{p, q}$, and so from (3.20) the result is proved.

We now pass to the case $p=q=\infty$. Let $f \in \boldsymbol{M}_{1 / v_{\lambda}}^{\infty}$ and $g \in \boldsymbol{M}_{v_{\lambda}}^{1}$. We have to prove that

$$
\langle f, g\rangle=\left\langle D_{\psi} C_{\varphi} f, g\right\rangle=\left\langle D_{\varphi} C_{\psi} f, g\right\rangle .
$$

From (3.18) and (3.19) we have that

$$
\left\langle D_{\psi} C_{\varphi} f, g\right\rangle=\left\langle f, D_{\varphi} C_{\psi} g\right\rangle
$$

from the previous point we have that $D_{\varphi} C_{\psi} g=g$ in $\boldsymbol{M}_{v_{\lambda}}^{1}$, so the first equality in (3.21) is proved. The other is similar.

Remark 3.16. Let $u \in \mathcal{S}_{\omega}^{\prime}\left(\mathbb{R}^{d}\right)$, and $\varphi, \psi \in \mathcal{S}_{\omega}\left(\mathbb{R}^{d}\right)$ as in Lemma 3.15. Then for every $\theta \in \mathcal{S}_{\omega}\left(\mathbb{R}^{d}\right)$ we have

$$
\langle u, \theta\rangle=\sum_{\sigma \in \Lambda}\langle u, \Pi(\sigma) \varphi\rangle\langle\Pi(\sigma) \psi, \theta\rangle .
$$

We have indeed that from Remark 3.6 there exists $\lambda<0$ such that $u \in \boldsymbol{M}_{m_{\lambda}}^{\infty}=\boldsymbol{M}_{1 / v_{\lambda}}^{\infty}$. Then, from Lemma 3.15, for every $g \in \boldsymbol{M}_{v_{\lambda}}^{1}$,

$$
\langle u, g\rangle=\sum_{\sigma \in \Lambda}\langle u, \Pi(\sigma) \varphi\rangle\langle\Pi(\sigma) \psi, g\rangle .
$$

From Proposition 3.9, the previous formula then holds for $g=\theta \in \mathcal{S}_{\omega}\left(\mathbb{R}^{d}\right)$, so we have (3.22).

We can now prove the main result of this section.

Theorem 3.17. If $u \in \mathcal{S}_{\omega}^{\prime}\left(\mathbb{R}^{d}\right)$ then

$$
\mathrm{WF}_{\omega}^{\prime}(u)=\mathrm{WF}_{\omega}^{G}(u) .
$$


Proof. The inclusion $\mathrm{WF}_{\omega}^{G}(u) \subseteq \mathrm{WF}_{\omega}^{\prime}(u)$ is trivial, so that we only have to prove that

$$
\mathrm{WF}_{\omega}^{\prime}(u) \subseteq \mathrm{WF}_{\omega}^{G}(u)
$$

Let $0 \neq z_{0} \notin \mathrm{WF}_{\omega}^{G}(u)$. So, there exists an open conic set $\Gamma \subset \mathbb{R}^{2 d} \backslash\{0\}$ containing $z_{0}$ such that (3.9) is satisfied. By Remark 3.16 we have that, for $\varphi \in \mathcal{S}_{\omega}\left(\mathbb{R}^{d}\right) \backslash\{0\}$ and $\tilde{\varphi}=S_{\varphi \varphi}^{-1} \varphi \in \mathcal{S}_{\omega}\left(\mathbb{R}^{d}\right)$ its dual window,

$$
\langle u, \psi\rangle=\sum_{\sigma \in \Lambda} V_{\varphi} u(\sigma)\langle\Pi(\sigma) \tilde{\varphi}, \psi\rangle \quad \forall \psi \in \mathcal{S}_{\omega}\left(\mathbb{R}^{d}\right)
$$

We denote

$$
\begin{aligned}
& u_{1}=\sum_{\sigma \in \Lambda \cap \Gamma} V_{\varphi} u(\sigma) \Pi(\sigma) \tilde{\varphi} \\
& u_{2}=\sum_{\sigma \in \Lambda \backslash \Gamma} V_{\varphi} u(\sigma) \Pi(\sigma) \tilde{\varphi} .
\end{aligned}
$$

Clearly $V_{\varphi} u(z)=V_{\varphi} u_{1}(z)+V_{\varphi} u_{2}(z)$. Denoting $\sigma=\left(\sigma_{1}, \sigma_{2}\right) \in \mathbb{R}^{d} \times \mathbb{R}^{d}$, by (2.12), (2.1), the subadditivity of $\omega$ and (2.14), we can estimate, for every $\alpha, \beta \in \mathbb{N}_{0}^{d}, \lambda, \mu>0$ :

$$
\begin{aligned}
& e^{-\lambda \varphi_{\omega}^{*}\left(\frac{|\alpha|}{\lambda}\right)} e^{-\mu \varphi_{\omega}^{*}\left(\frac{|\beta|}{\mu}\right)}\left|x^{\beta} \partial^{\alpha} u_{1}(x)\right| \\
& \leq \sum_{\sigma \in \Lambda \cap \Gamma}\left|V_{\varphi} u(\sigma)\right| \cdot\left|x^{\beta} \partial^{\alpha}\left(e^{i\left\langle\sigma_{2}, x\right\rangle} \tilde{\varphi}\left(x-\sigma_{1}\right)\right)\right| e^{-\lambda \varphi_{\omega}^{*}\left(\frac{|\alpha|}{\lambda}\right)} e^{-\mu \varphi_{\omega}^{*}\left(\frac{|\beta|}{\mu}\right)} \\
& \leq \sum_{\sigma \in \Lambda \cap \Gamma}\left|V_{\varphi} u(\sigma)\right| \sum_{\gamma \leq \alpha}\left(\begin{array}{l}
\alpha \\
\gamma
\end{array}\right) 2^{-|\alpha|}|x|^{|\beta|} e^{-\mu \varphi_{\omega}^{*}\left(\frac{|\beta|}{\mu}\right)}\left\langle\sigma_{2}\right\rangle^{|\alpha-\gamma|}\left|\partial^{\gamma} \tilde{\varphi}\left(x-\sigma_{1}\right)\right| e^{-\lambda \varphi_{\omega}^{*}\left(\frac{|\alpha|}{\lambda}\right) 2^{|\alpha|}} \\
& \leq C_{\mu} \sum_{\sigma \in \Lambda \cap \Gamma}\left|V_{\varphi} u(\sigma)\right| \sum_{\gamma \leq \alpha}\left(\begin{array}{l}
\alpha \\
\gamma
\end{array}\right) 2^{-|\alpha|} e^{\mu \omega(x)}\left|\partial^{\gamma} \tilde{\varphi}\left(x-\sigma_{1}\right)\right|\left\langle\sigma_{2}\right\rangle^{|\alpha-\gamma|} e^{-3 \lambda \varphi_{\omega}^{*}\left(\frac{|\alpha|}{3 \lambda}\right)} \\
& \leq C_{\mu} \sum_{\sigma \in \Lambda \cap \Gamma}\left|V_{\varphi} u(\sigma)\right| \sum_{\gamma \leq \alpha}\left(\begin{array}{l}
\alpha \\
\gamma
\end{array}\right) 2^{-|\alpha|} e^{\mu \omega\left(\sigma_{1}\right)} e^{\mu \omega\left(x-\sigma_{1}\right)}\left|\partial^{\gamma} \tilde{\varphi}\left(x-\sigma_{1}\right)\right|\left\langle\sigma_{2}\right\rangle^{|\alpha-\gamma|} e^{-3 \lambda \varphi_{\omega}^{*}\left(\frac{|\alpha|}{3 \lambda}\right)} \\
& \leq C_{\lambda^{\prime}, \mu} \sum_{\sigma \in \Lambda \cap \Gamma}\left|V_{\varphi} u(\sigma)\right| \sum_{\gamma \leq \alpha}\left(\begin{array}{l}
\alpha \\
\gamma
\end{array}\right) 2^{-|\alpha|} e^{\mu \omega\left(\sigma_{1}\right)} e^{\lambda^{\prime} \varphi_{\omega}^{*}\left(\frac{|\gamma|}{\lambda^{\prime}}\right)-3 \lambda \varphi_{\omega}^{*}\left(\frac{|\alpha|}{3 \lambda}\right)}\left\langle\sigma_{2}\right\rangle^{|\alpha-\gamma|}
\end{aligned}
$$

for some $C_{\mu}, C_{\lambda^{\prime}, \mu}>0$. 
For $\lambda^{\prime}=6 \lambda$ we apply [5, Prop. 2.1(g)], then (2.12) and (3.9), and finally obtain, for some constants depending on $\lambda$ and $\mu$, and $m>0$ big enough:

$$
\begin{aligned}
& e^{-\lambda \varphi_{\omega}^{*}\left(\frac{|\alpha|}{\lambda}\right)} e^{-\mu \varphi_{\omega}^{*}\left(\frac{|\beta|}{\mu}\right)}\left|x^{\beta} \partial^{\alpha} u_{1}(x)\right| \\
& \leq C_{\lambda, \mu} \sum_{\sigma \in \Lambda \cap \Gamma}\left|V_{\varphi} u(\sigma)\right| \sum_{\gamma \leq \alpha}\left(\begin{array}{l}
\alpha \\
\gamma
\end{array}\right) 2^{-|\alpha|} e^{\mu \omega\left(\sigma_{1}\right)} e^{-6 \lambda \varphi_{\omega}^{*}\left(\frac{|\alpha-\gamma|}{6 \lambda}\right)}\left\langle\sigma_{2}\right\rangle^{|\alpha-\gamma|} \\
& \leq C_{\lambda, \mu} \sum_{\sigma \in \Lambda \cap \Gamma}\left|V_{\varphi} u(\sigma)\right| \sum_{\gamma \leq \alpha}\left(\begin{array}{l}
\alpha \\
\gamma
\end{array}\right) 2^{-|\alpha|} e^{\mu \omega\left(\sigma_{1}\right)} e^{6 \lambda \omega\left(\left\langle\sigma_{2}\right\rangle\right)} \\
& \leq C_{\lambda, \mu} \sum_{\sigma \in \Lambda \cap \Gamma}\left|V_{\varphi} u(\sigma)\right| e^{(\mu+6 \lambda) \omega(\langle\sigma\rangle)+m \omega(\langle\sigma\rangle)} e^{-m \omega(\langle\sigma\rangle)} \\
& \leq C_{\lambda, \mu}^{\prime} \sum_{\sigma \in \Lambda \cap \Gamma} e^{-m \omega(\langle\sigma\rangle)} \leq C_{\lambda, \mu}^{\prime \prime}, \quad x \in \mathbb{R}^{d} .
\end{aligned}
$$

This proves that $u_{1} \in \mathcal{S}_{\omega}\left(\mathbb{R}^{d}\right)$ (here, we consider the seminorms given in (2.2)). Therefore, from Theorem 2.7, $V_{\varphi} u_{1} \in \mathcal{S}_{\omega}\left(\mathbb{R}^{2 d}\right)$ and for every $\lambda>0$ there is a constant $C_{\lambda}>0$ such that

$$
e^{\lambda \omega(z)}\left|V_{\varphi} u_{1}(z)\right| \leq C_{\lambda} \quad \forall z \in \mathbb{R}^{2 d} .
$$

Let us now fix an open conic set $\Gamma^{\prime} \subset \mathbb{R}^{2 d} \backslash\{0\}$ containing $z_{0}$ and such that $\overline{\Gamma^{\prime} \cap S_{2 d-1}} \subseteq \Gamma$. Then

$$
\inf _{\substack{0 \neq \sigma \in \Lambda \backslash \Gamma \\ z \in \Gamma^{\prime}}}\left|\frac{\sigma}{|\sigma|}-z\right|=\varepsilon>0
$$

and $|\sigma-z| \geq \varepsilon|\sigma|$ for $0 \neq \sigma \in \Lambda \backslash \Gamma$ and $z \in \Gamma^{\prime}$.

From the subadditivity of $\omega$ we have

$$
\begin{aligned}
e^{\lambda \omega(z)}\left|V_{\varphi} u_{2}(z)\right| & \leq \sum_{\sigma \in \Lambda \backslash \Gamma} e^{\lambda \omega(\sigma)+\lambda \omega(z-\sigma)}\left|V_{\varphi} u_{2}(\sigma)\right| \cdot|\langle\Pi(\sigma) \tilde{\varphi}, \Pi(z) \varphi\rangle| \\
& \leq C \sum_{\sigma \in \Lambda \backslash \Gamma} e^{(\lambda+\bar{\lambda}) \omega(\sigma)} e^{\lambda \omega(z-\sigma)}\left|V_{\varphi} \tilde{\varphi}(z-\sigma)\right|
\end{aligned}
$$

for some $C, \bar{\lambda}>0$, because of Theorem 2.5 and since ([18, pg 41])

$$
|\langle\Pi(\sigma) \tilde{\varphi}, \Pi(z) \varphi\rangle|=\left|e^{-i\left\langle\sigma_{1}, z_{2}-\sigma_{2}\right\rangle} V_{\varphi} \tilde{\varphi}(z-\sigma)\right|=\left|V_{\varphi} \tilde{\varphi}(z-\sigma)\right| .
$$

Since $\tilde{\varphi} \in \mathcal{S}_{\omega}\left(\mathbb{R}^{d}\right)$, from Theorem 2.7 we have that for every $\mu>0$ there is a constant $C_{\mu}>0$ such that

$$
\left|V_{\varphi} \tilde{\varphi}(z-\sigma)\right| \leq C_{\mu} e^{-\mu \omega(z-\sigma)}
$$

and hence, substituting in (3.26):

$$
e^{\lambda \omega(z)}\left|V_{\varphi} u_{2}(z)\right| \leq C C_{\mu} \sum_{\sigma \in \Lambda \backslash \Gamma} e^{(\lambda+\bar{\lambda}) \omega(\sigma)} e^{(\lambda-\mu) \omega(z-\sigma)} .
$$


However, for $z \in \Gamma^{\prime}$ and $\sigma \in \Lambda \backslash \Gamma$ we have $|\sigma-z| \geq \varepsilon|\sigma|$ and therefore, by the subadditivity of $\omega$, we have that

$$
\omega(\sigma)=\omega\left(\frac{\varepsilon|\sigma|}{\varepsilon}\right) \leq\left(\left[\frac{1}{\varepsilon}\right]+1\right) \omega(\varepsilon|\sigma|) \leq\left(\left[\frac{1}{\varepsilon}\right]+1\right) \omega(z-\sigma) .
$$

Substituting in (3.28) we obtain, for $M=([1 / \varepsilon]+1)^{-1}$ and $\mu>\lambda$ sufficiently large:

$$
e^{\lambda \omega(z)}\left|V_{\varphi} u_{2}(z)\right| \leq C C_{\mu} \sum_{\sigma \in \Lambda \backslash \Gamma} e^{(\lambda+\bar{\lambda}+\lambda M-\mu M) \omega(\sigma)} \leq C_{\lambda}, \quad z \in \Gamma^{\prime},
$$

for some $C_{\lambda}>0$.

From (3.24) and (3.29) we finally deduce

$$
\sup _{z \in \Gamma^{\prime}} e^{\lambda \omega(z)}\left|V_{\varphi} u(z)\right|<+\infty, \quad \lambda>0
$$

and hence $z_{0} \notin \mathrm{WF}_{\omega}^{\prime}(u)$.

From Theorem 3.17, in what follows we use $\mathrm{WF}_{\omega}^{\prime}(u)$ for $\mathrm{WF}_{\omega}^{G}(u)$ and any $u \in \mathcal{S}_{\omega}^{\prime}\left(\mathbb{R}^{d}\right)$.

Proposition 3.18. For every $u \in \mathcal{S}_{\omega}^{\prime}\left(\mathbb{R}^{d}\right)$ we have $\mathrm{WF}_{\omega}^{\prime}(u)=\emptyset$ if and only if $u \in \mathcal{S}_{\omega}\left(\mathbb{R}^{d}\right)$.

Proof. Suppose that $u \in \mathcal{S}_{\omega}\left(\mathbb{R}^{d}\right)$, and fix a window function $\varphi \in \mathcal{S}_{\omega}\left(\mathbb{R}^{d}\right) \backslash\{0\}$; from Theorem 2.7 we have that for every $\lambda>0$ there exists $C_{\lambda}>0$ such that

$$
\left|V_{\varphi} u(z)\right| \leq C_{\lambda} e^{-\lambda \omega(z)}, \quad \forall z \in \mathbb{R}^{2 d}
$$

Then for every open conic set $\Gamma \subseteq \mathbb{R}^{2 d} \backslash\{0\}$ condition $(3.1)$ holds, so $\operatorname{WF}_{\omega}^{\prime}(u)=\emptyset$.

Suppose now that $\mathrm{WF}_{\omega}^{\prime}(u)=\emptyset$. From Definition 3.1 we have that for every $\bar{z} \in \mathbb{R}^{2 d} \backslash\{0\}$ there exists an open conic set $\Gamma_{\bar{z}} \subseteq \mathbb{R}^{2 d} \backslash\{0\}$ containing $\bar{z}$ such that for every $\lambda>0$ there exists $C_{\lambda, \bar{z}}>0$ satisfying

$$
\left|V_{\varphi} u(z)\right| \leq C_{\lambda, \bar{z}} e^{-\lambda \omega(z)} \quad \forall z \in \Gamma_{\bar{z}}
$$

Let $\Upsilon_{\bar{z}}=\Gamma_{\bar{z}} \cap S_{2 d-1}$. We have that $\left\{\Upsilon_{\bar{z}}, \bar{z} \in \mathbb{R}^{2 d} \backslash\{0\}\right\}$ is an open covering of $S_{2 d-1}$; since $S_{2 d-1}$ is compact and $\Gamma_{\bar{z}}$ is conic, there exist $z_{1}, \ldots, z_{k} \in \mathbb{R}^{2 d} \backslash\{0\}$ such that

$$
\Gamma_{z_{1}} \cup \cdots \cup \Gamma_{z_{k}}=\mathbb{R}^{2 d} \backslash\{0\}
$$

We then have that for every $\lambda>0$,

$$
\left|V_{\varphi} u(z)\right| \leq C_{\lambda} e^{-\lambda \omega(z)} \quad \forall z \in \mathbb{R}^{2 d},
$$

where $C_{\lambda}=\max \left\{C_{\lambda, z_{1}}, \ldots, C_{\lambda, z_{k}},\left|V_{\varphi} u(0)\right| e^{\lambda \omega(0)}\right\}$. From Theorem 2.7 we finally have $u \in$ $\mathcal{S}_{\omega}\left(\mathbb{R}^{d}\right)$.

We now prove that the wave front set $\mathrm{WF}_{\omega}^{\prime}$ is not affected by the phase-space shift operator.

Proposition 3.19. For every $w=(y, \eta) \in \mathbb{R}^{2 d}$ and for every $u \in \mathcal{S}_{\omega}^{\prime}\left(\mathbb{R}^{d}\right)$ we have

$$
\mathrm{WF}_{\omega}^{\prime}(\Pi(w) u)=\mathrm{WF}_{\omega}^{\prime}(u)
$$


Proof. Since $\Pi(w)=M_{\eta} T_{y}$, it is enough to prove that translation and modulation do not affect the wave front set. Concerning translation, we have that for $z=(x, \xi) \in \mathbb{R}^{2 d}$,

$$
V_{\varphi}\left(T_{y} u\right)(z)=\left\langle T_{y} u, \Pi(z) \varphi\right\rangle=\left\langle u, T_{-y} \Pi(z) \varphi\right\rangle=e^{-i\langle y, \xi\rangle} V_{T_{-y} \varphi} u
$$

writing $\psi=T_{-y} \varphi \in \mathcal{S}_{\omega}\left(\mathbb{R}^{d}\right)$ we have that

$$
\left|V_{\varphi}\left(T_{y} u\right)(z)\right|=\left|V_{\psi} u(z)\right|,
$$

and since the wave front set does not depend on the window (Proposition 3.2) we have $\mathrm{WF}_{\omega}^{\prime}\left(T_{y} u\right)=\mathrm{WF}_{\omega}^{\prime}(u)$. Concerning modulation, we have

$$
V_{\varphi}\left(M_{\eta} u\right)(z)=\left\langle M_{\eta} u, \Pi(z) \varphi\right\rangle=\left\langle u, M_{-\eta} \Pi(z) \varphi\right\rangle=e^{i\langle\eta, x\rangle} V_{M_{-\eta} \varphi} u(z) ;
$$

then, writing $\theta=M_{-\eta} \varphi \in \mathcal{S}_{\omega}\left(\mathbb{R}^{d}\right)$, we get

$$
\left|V_{\varphi}\left(M_{\eta} u\right)(z)\right|=\left|V_{\theta} u(z)\right|
$$

and as before we conclude that $\mathrm{WF}_{\omega}^{\prime}\left(M_{\eta} u\right)=\mathrm{WF}_{\omega}^{\prime}(u)$.

The results obtained in Sections 2 and 3 are true in the quasi-analytic case also, i.e. when we consider that $\omega(t)=o(t)$, as $t \rightarrow+\infty$, instead of condition $(\beta)$ of Definition 2.1. However, in the following we will consider weights satisfying $(\beta)$, i.e. there are compactly supported functions in $\mathcal{S}_{\omega}\left(\mathbb{R}^{d}\right)$.

\section{Applications to (pseudo-)differential operators}

In this section we analyze the action of several operators of pseudo-differential (or differential) type on the global wave front set $\mathrm{WF}_{\omega}^{\prime}(u)$ of $u \in \mathcal{S}_{\omega}^{\prime}\left(\mathbb{R}^{d}\right)$. In particular, we obtain regularity results for pseudo-differential operators of infinite order in the Beurling setting. Note that, in the classical Schwartz space $\mathcal{S}\left(\mathbb{R}^{d}\right)$, Rodino-Wahlberg treat in [35] pseudo-differential operators with symbols of polynomial growth.

In order to study the behaviour of the $\omega$-wave front set of pseudo-differential operators of infinite order we need nuclearity of $\mathcal{S}_{\omega}$ to apply the kernel theorem. It is known that $\mathcal{S}_{\omega}$ is nuclear for many weight functions $\omega$. For example, whenever they satisfy the following condition:

$$
\exists H>1 \forall t \geq 0,2 \omega(t) \leq \omega(H t)+H .
$$

Bonet, Meise and Melikhov [9] proved that under such a condition the classes of ultradifferentiable functions defined by sequences in the sense of Komatsu satisfying the standard conditions $(M 0),(M 1),(M 2)$ and (M3), and the classes defined by weight functions in the sense of Braun, Meise and Taylor [11] coincide. Hence, under condition (4.1) our results are true also for spaces defined by sequences instead of weights (see, for instance, Langenbruch [27] for a complete study of the structure of many global weighted spaces of (ultra)differentiable functions and ultradistributions defined by sequences in the sense of Komatsu).

First, we state the following property:

Lemma 4.1. If the weight function $\omega$ satisfies (4.1) then

$$
\log t=o(\omega(t)), \quad \text { as } t \rightarrow+\infty .
$$


Proof. Let $H>1$ be the constant of (4.1). We fix $c>0$ such that $\omega(c)>H$. For $t \geq c H$ there exists $m \in \mathbb{N}$ such that

$$
c H^{m} \leq t \leq c H^{m+1}
$$

By (4.1) we have, for all $x \geq 0$,

$$
2^{2} \omega(x) \leq 2 \omega(H x)+2 H \leq \omega\left(H^{2} x\right)+2 H+H .
$$

Hence, by induction on $k \in \mathbb{N}$, we obtain

$$
2^{k} \omega(x) \leq \omega\left(H^{k} x\right)+\left(2^{k-1}+2^{k-2}+\cdots+1\right) H .
$$

Therefore,

$$
\begin{aligned}
\lim _{t \rightarrow \infty} \frac{\log t}{\omega(t)} & \leq \lim _{\substack{m \rightarrow \infty \\
m \in \mathbb{N}}} \frac{\log \left(c H^{m+1}\right)}{\omega\left(c H^{m}\right)} \leq \lim _{\substack{m \rightarrow \infty \\
m \in \mathbb{N}}} \frac{(m+1) \log H+\log c}{2^{m}\left[\omega(c)-\frac{H}{2}-\frac{H}{2^{2}}-\cdots-\frac{H}{2^{m}}\right]} \\
& =\lim _{\substack{m \rightarrow \infty \\
m \in \mathbb{N}}} \frac{(m+1) \log H+\log c}{2^{m}\left[\omega(c)-H+\frac{H}{2^{m}}\right]}=0 .
\end{aligned}
$$

We start by defining the following symbol class.

Definition 4.2. For $m \in \mathbb{R}$ we define

$$
\begin{aligned}
S_{\omega}^{m}:=\left\{a \in C^{\infty}\left(\mathbb{R}^{2 d}\right): \forall \lambda, \mu>0 \exists C_{\lambda, \mu}>0\right. \text { such that } \\
\left.\quad\left|\partial_{x}^{\alpha} \partial_{\xi}^{\beta} a(x, \xi)\right| \leq C_{\lambda, \mu} e^{\lambda \varphi_{\omega}^{*}\left(\frac{|\alpha|}{\lambda}\right)} e^{\mu \varphi_{\omega}^{*}\left(\frac{|\beta|}{\mu}\right)} e^{m \omega(\xi)}, \forall(x, \xi) \in \mathbb{R}^{2 d}, \alpha, \beta \in \mathbb{N}_{0}^{d}\right\} .
\end{aligned}
$$

Let us remark that when $\omega(t)=\log (1+t)$ then $S_{\omega}^{m}$ contains the classical Hörmander symbol classes of global type and finite order $S_{\rho, 0}^{m}$, for all $\rho \in[0,1]$, and, in particular, it coincides with $S_{0,0}^{m}$ (see [25] and the arguments of [15, Example $\left.2.11(1)\right]$ ). However, in the present section, we are not considering this extreme case by Lemma 4.1. We extend the results of [35] for symbols of type $(0,0)$ with infinite order.

Then we consider the Kohn-Nirenberg quantization defined by

$$
a(x, D) f(x):=(2 \pi)^{-d} \int_{\mathbb{R}^{d}} e^{i\langle x, \xi\rangle} a(x, \xi) \widehat{f}(\xi) d \xi, \quad a \in S_{\omega}^{m}, f \in \mathcal{S}_{\omega}\left(\mathbb{R}^{d}\right) .
$$

The above Kohn-Nirenberg quantization is well defined since $\widehat{f} \in \mathcal{S}_{\omega}\left(\mathbb{R}^{d}\right)$ and hence for every $\lambda>0$ there exists $C_{\lambda}>0$ such that

$$
|a(x, \xi)| \cdot|\widehat{f}(\xi)| \leq e^{m \omega(\xi)} C_{\lambda} e^{-\lambda \omega(\xi)}
$$

which is integrable in $\mathbb{R}^{d}$ if we choose $\lambda>0$ sufficiently large. Moreover,

$$
a(x, D): \mathcal{S}_{\omega} \longrightarrow \mathcal{S}^{\prime} \subseteq \mathcal{S}_{\omega}^{\prime} .
$$

If $\mathcal{S}_{\omega}$ is nuclear, we can apply the kernel theorem to the linear operator

$$
V_{\varphi} a(x, D) V_{\varphi}^{*}: \mathcal{S}_{\omega}\left(\mathbb{R}^{2 d}\right) \longrightarrow \mathcal{S}_{\omega}^{\prime}\left(\mathbb{R}^{2 d}\right)
$$

and find a unique distribution $K \in \mathcal{S}_{\omega}^{\prime}\left(\mathbb{R}^{4 d}\right)$ such that

$$
V_{\varphi} a(x, D) V_{\varphi}^{*} F\left(y^{\prime}, \eta^{\prime}\right)=(2 \pi)^{d} \int_{\mathbb{R}^{2 d}} K\left(y^{\prime}, \eta^{\prime} ; y, \eta\right) F(y, \eta) d y d \eta \quad \forall F \in \mathcal{S}_{\omega}\left(\mathbb{R}^{2 d}\right)
$$


in the sense that

$$
\left\langle V_{\varphi} a(x, D) V_{\varphi}^{*} F, G\right\rangle=(2 \pi)^{d}\left\langle K\left(y^{\prime}, \eta^{\prime} ; y, \eta\right), G\left(y^{\prime}, \eta^{\prime}\right) \overline{F(y, \eta)}\right\rangle \quad \forall G \in \mathcal{S}_{\omega}\left(\mathbb{R}^{2 d}\right) .
$$

If $u \in \mathcal{S}_{\omega}\left(\mathbb{R}^{d}\right)$ and $F=V_{\varphi} u \in \mathcal{S}_{\omega}\left(\mathbb{R}^{2 d}\right)$ for $\varphi \in \mathcal{S}_{\omega}\left(\mathbb{R}^{d}\right)$ with $\|\varphi\|_{L^{2}}=1$, then, from $(2.22)$,

$$
\begin{aligned}
V_{\varphi} a(x, D) u\left(y^{\prime}, \eta^{\prime}\right) & =(2 \pi)^{-d} V_{\varphi} a(x, D) V_{\varphi}^{*} V_{\varphi} u\left(y^{\prime}, \eta^{\prime}\right) \\
& =\int_{\mathbb{R}^{2 d}} K\left(y^{\prime}, \eta^{\prime} ; y, \eta\right) V_{\varphi} u(y, \eta) d y d \eta
\end{aligned}
$$

and we can compute the kernel directly:

Lemma 4.3. For $a \in S_{\omega}^{m}, \varphi \in \mathcal{S}_{\omega}\left(\mathbb{R}^{d}\right)$ with $\|\varphi\|_{L^{2}}=1$ and $u \in \mathcal{S}_{\omega}\left(\mathbb{R}^{d}\right)$ we have that

$$
V_{\varphi}(a(x, D) u)\left(z^{\prime}\right)=\int_{\mathbb{R}^{2 d}} K\left(z^{\prime}, z\right) V_{\varphi} u(z) d z,
$$

where, for all $z=(y, \eta), z^{\prime}=\left(y^{\prime}, \eta^{\prime}\right) \in \mathbb{R}^{2 d}$,

$$
K\left(z^{\prime}, z\right)=(2 \pi)^{-2 d} e^{i\langle y, \eta\rangle} \int_{\mathbb{R}^{2 d}} e^{i\left(\langle x, \xi\rangle-\langle y, \xi\rangle-\left\langle x, \eta^{\prime}\right\rangle\right)} a(x, \xi) \widehat{\varphi}(\xi-\eta) \overline{\varphi\left(x-y^{\prime}\right)} d x d \xi .
$$

Proof. Let $F \in \mathcal{S}_{\omega}\left(\mathbb{R}^{2 d}\right)$ and consider the Kohn-Nirenberg quantization (4.3) of $V_{\varphi}^{*} F \in \mathcal{S}_{\omega}\left(\mathbb{R}^{d}\right)$ :

$$
a(x, D) V_{\varphi}^{*} F(x)=(2 \pi)^{-d} \int_{\mathbb{R}^{d}} e^{i\langle x, \xi\rangle} a(x, \xi) \widehat{V_{\varphi}^{*} F}(\xi) d \xi .
$$

Then, by the definition of short-time Fourier transform and (4.3):

$$
\begin{aligned}
& V_{\varphi} a(x, D) V_{\varphi}^{*} F\left(y^{\prime}, \eta^{\prime}\right)=\int_{\mathbb{R}^{d}}\left(a(x, D) V_{\varphi}^{*} F\right)(x) \overline{\varphi\left(x-y^{\prime}\right)} e^{-i\left\langle x, \eta^{\prime}\right\rangle} d x \\
& =(2 \pi)^{-d} \int_{\mathbb{R}^{d}} \int_{\mathbb{R}^{d}} e^{i\langle x, \xi\rangle} a(x, \xi) \widehat{V_{\varphi}^{*} F}(\xi) \overline{\varphi\left(x-y^{\prime}\right)} e^{-i\left\langle x, \eta^{\prime}\right\rangle} d \xi d x .
\end{aligned}
$$

So, fixed $x, \xi$ we have, by $(2.23)$,

$$
\begin{aligned}
& e^{i\langle x, \xi\rangle} a(x, \xi) \widehat{V_{\varphi}^{*} F}(\xi) \overline{\varphi\left(x-y^{\prime}\right)} e^{-i\left\langle x, \eta^{\prime}\right\rangle} \\
& =\int_{\mathbb{R}^{d}} e^{i\langle x, \xi\rangle} a(x, \xi) V_{\varphi}^{*} F\left(x^{\prime}\right) e^{-i\left\langle x^{\prime}, \xi\right\rangle} \overline{\varphi\left(x-y^{\prime}\right)} e^{-i\left\langle x, \eta^{\prime}\right\rangle} d x^{\prime} \\
& =\int_{\mathbb{R}^{d}} \int_{\mathbb{R}^{2 d}} e^{i\langle x, \xi\rangle} a(x, \xi) F(y, \eta) e^{i\left\langle x^{\prime}, \eta\right\rangle} \varphi\left(x^{\prime}-y\right) \\
& \cdot e^{-i\left\langle x^{\prime}, \xi\right\rangle} \overline{\varphi\left(x-y^{\prime}\right)} e^{-i\left\langle x, \eta^{\prime}\right\rangle} d y d \eta d x^{\prime} .
\end{aligned}
$$

Since $a \in S_{\omega}^{m}, F \in \mathcal{S}_{\omega}\left(\mathbb{R}^{2 d}\right)$ and $\varphi \in \mathcal{S}_{\omega}\left(\mathbb{R}^{d}\right)$, we have that for every $\lambda_{1}, \lambda_{2}, \lambda_{3}>0$ there exists a constant $C_{\lambda}>0$ such that, by the subaddititvity of $\omega$ :

$$
\begin{aligned}
& \left|a(x, \xi) F(y, \eta) \varphi\left(x^{\prime}-y\right) \overline{\varphi\left(x-y^{\prime}\right)}\right| \\
& \leq C_{\lambda} e^{m \omega(\xi)} e^{-\lambda_{1} \omega(y, \eta)} e^{-\lambda_{2} \omega\left(x^{\prime}-y\right)} e^{-\lambda_{3} \omega\left(x-y^{\prime}\right)} \\
& \leq C_{\lambda} e^{m \omega(\xi)} e^{-\frac{\lambda_{1}}{2} \omega(y)} e^{-\frac{\lambda_{1}}{2} \omega(\eta)} e^{-\lambda_{2} \omega\left(x^{\prime}\right)+\lambda_{2} \omega(y)} e^{-\lambda_{3} \omega(x)+\lambda_{3} \omega\left(y^{\prime}\right)} .
\end{aligned}
$$


Choosing $\lambda_{1}>2 \lambda_{2}>0$ sufficiently large we can apply Fubini's theorem with respect to the variables $y, \eta$ and $x^{\prime}$, obtaining:

$$
\begin{aligned}
& e^{i\langle x, \xi\rangle} a(x, \xi) \widehat{V_{\varphi}^{*} F}(\xi) \overline{\varphi\left(x-y^{\prime}\right)} e^{-i\left\langle x, \eta^{\prime}\right\rangle} \\
& =\int_{\mathbb{R}^{2 d}} e^{i\langle x, \xi\rangle} a(x, \xi) F(y, \eta) \\
& \cdot\left(\int_{\mathbb{R}^{d}} e^{i\left\langle x^{\prime}, \eta\right\rangle} \varphi\left(x^{\prime}-y\right) e^{-i\left\langle x^{\prime}, \xi\right\rangle} d x^{\prime}\right) \overline{\varphi\left(x-y^{\prime}\right)} e^{-i\left\langle x, \eta^{\prime}\right\rangle} d y d \eta \\
& =\int_{\mathbb{R}^{2 d}} e^{i\langle x, \xi\rangle} a(x, \xi) F(y, \eta) \\
& \cdot\left(\int_{\mathbb{R}^{d}} e^{i\langle y+s, \eta\rangle} e^{-i\langle y+s, \xi\rangle} \varphi(s) d s\right) \overline{\varphi\left(x-y^{\prime}\right)} e^{-i\left\langle x, \eta^{\prime}\right\rangle} d y d \eta \\
& =\int_{\mathbb{R}^{2 d}} e^{i\langle x, \xi\rangle} a(x, \xi) F(y, \eta) e^{i\langle y, \eta\rangle} e^{-i\langle y, \xi\rangle} \\
& \cdot\left(\int_{\mathbb{R}^{d}} e^{-i\langle s, \xi-\eta\rangle} \varphi(s) d s\right) \overline{\varphi\left(x-y^{\prime}\right)} e^{-i\left\langle x, \eta^{\prime}\right\rangle} d y d \eta \\
& =\int_{\mathbb{R}^{2 d}} e^{i\langle x, \xi\rangle} a(x, \xi) F(y, \eta) e^{i\langle y, \eta\rangle} e^{-i\langle y, \xi\rangle} \\
& \widehat{\varphi}(\xi-\eta) \overline{\varphi\left(x-y^{\prime}\right)} e^{-i\left\langle x, \eta^{\prime}\right\rangle} d y d \eta
\end{aligned}
$$

Since $a \in S_{\omega}^{m}, F \in \mathcal{S}_{\omega}\left(\mathbb{R}^{2 d}\right)$ and $\varphi \in \mathcal{S}_{\omega}\left(\mathbb{R}^{d}\right)$, for every $\mu_{1}, \mu_{2}, \mu_{3}>0$ there exists a constant $C_{\mu}>0$ such that, by the subadditivity of $\omega$,

$$
\begin{aligned}
& \left|a(x, \xi) F(y, \eta) \widehat{\varphi}(\xi-\eta) \overline{\varphi\left(x-y^{\prime}\right)}\right| \\
& \quad \leq C_{\mu} e^{m \omega(\xi)} e^{-\mu_{1} \omega(y)} e^{-\mu_{1} \omega(\eta)} e^{-\mu_{2} \omega(\xi)+\mu_{2} \omega(\eta)} e^{-\mu_{3} \omega(x)+\mu_{3} \omega\left(y^{\prime}\right)},
\end{aligned}
$$

so that, for $\mu_{3}, \mu_{1}>\mu_{2}$ sufficiently large, the above function is integrable in $\mathbb{R}_{(x, \xi, \eta, y)}^{4 d}$ and substituting (4.9) into (4.8) we can apply Fubini's theorem to obtain:

$$
\begin{array}{rl}
V_{\varphi} a(x, D) V_{\varphi}^{*} F\left(y^{\prime}, \eta^{\prime}\right)= & \\
=(2 \pi)^{-d} \int_{\mathbb{R}^{2 d}} & F(y, \eta) e^{i\langle y, \eta\rangle} \\
& \cdot\left(\int_{\mathbb{R}^{2 d}} e^{i\left(\langle x, \xi\rangle-\langle y, \xi\rangle-\left\langle x, \eta^{\prime}\right\rangle\right)} a(x, \xi) \widehat{\varphi}(\xi-\eta) \overline{\varphi\left(x-y^{\prime}\right)} d x d \xi\right) d y d \eta .
\end{array}
$$

Applying the above result to $F=V_{\varphi} u$ for some $u \in \mathcal{S}_{\omega}\left(\mathbb{R}^{d}\right)$, since $\|\varphi\|_{L^{2}}=1$ and hence $V_{\varphi}^{*} F=V_{\varphi}^{*} V_{\varphi} u=(2 \pi)^{d} u$ by $(2.22)$, we have

$$
V_{\varphi}(a(x, D) u)\left(y^{\prime}, \eta^{\prime}\right)=\int_{\mathbb{R}^{2 d}} K\left(y^{\prime}, \eta^{\prime} ; y, \eta\right) V_{\varphi} u(y, \eta) d y d \eta
$$

for

$$
K\left(y^{\prime}, \eta^{\prime} ; y, \eta\right)=(2 \pi)^{-2 d} e^{i\langle y, \eta\rangle} \int_{\mathbb{R}^{2 d}} e^{i\left(\langle x, \xi\rangle-\langle y, \xi\rangle-\left\langle x, \eta^{\prime}\right\rangle\right)} a(x, \xi) \widehat{\varphi}(\xi-\eta) \overline{\varphi\left(x-y^{\prime}\right)} d x d \xi
$$

which concludes the proof of the lemma. 
In the next result the following property on the weight function $\omega$, which can be proved as in [8, Lemma 4.7(ii)] (for instance), will be useful: from for every $\sigma, \mu>0$ and $t \geq 1$,

$$
\inf _{j \in \mathbb{N}_{0}} t^{-\sigma j} e^{\mu \varphi_{\omega}^{*}\left(\frac{\sigma j}{\mu}\right)} \leq e^{-\left(\mu-\frac{\sigma}{b}\right) \omega(t)-\frac{\sigma a}{b}}
$$

where $a \in \mathbb{R}$ and $b>0$ are the constants of condition $(\gamma)$ in Definition 2.1.

Proposition 4.4. If $a \in S_{\omega}^{m}, m \in \mathbb{R}$ and $K \in C^{\infty}\left(\mathbb{R}^{4 d}\right)$ is defined by (4.7), then for every $\lambda>0$ there exists a constant $C_{\lambda}>0$ such that

$$
\left|K\left(z^{\prime}, z\right)\right| \leq C_{\lambda} e^{-\lambda \omega\left(y-y^{\prime}\right)} e^{(m-\lambda) \omega\left(\eta-\eta^{\prime}\right)} e^{m \omega\left(\eta^{\prime}\right)}, \quad z=(y, \eta), z^{\prime}=\left(y^{\prime}, \eta^{\prime}\right) \in \mathbb{R}^{2 d} .
$$

Moreover, if $a(z)=0$ for $z \in \Gamma \backslash \overline{B(0, R)}$ for an open conic set $\Gamma \subseteq \mathbb{R}^{2 d} \backslash\{0\}$ and for some $R>0$ (here $B(0, R)$ is the ball of center 0 and radius $R$ in $\mathbb{R}^{2 d}$ ), then for every open conic set $\Gamma^{\prime} \subseteq \mathbb{R}^{2 d} \backslash\{0\}$ such that $\overline{\Gamma^{\prime} \cap S_{2 d-1}} \subseteq \Gamma$ we have that for every $\lambda>0$ there exists a constant $C_{\lambda}>0$ such that for all $z^{\prime}=\left(y^{\prime}, \eta^{\prime}\right) \in \Gamma^{\prime}$ and $z=(y, \eta) \in \mathbb{R}^{2 d}$,

$$
\left|K\left(z^{\prime}, z\right)\right| \leq C_{\lambda} e^{-\lambda \omega\left(y-y^{\prime}\right)} e^{-\lambda \omega\left(\eta-\eta^{\prime}\right)} e^{-2 \lambda \omega\left(y^{\prime}\right)} e^{-2 \lambda \omega\left(\eta^{\prime}\right)} .
$$

Proof. By the linear change of variables $\xi^{\prime}=\xi-\eta$ and $x^{\prime}=x-y^{\prime}$ in (4.7) we have

$$
\begin{aligned}
K\left(z^{\prime}, z\right)= & (2 \pi)^{-2 d} e^{i\langle y, \eta\rangle} \int_{\mathbb{R}^{2 d}} e^{i\left(\left\langle x^{\prime}+y^{\prime}, \xi^{\prime}+\eta\right\rangle-\left\langle y, \xi^{\prime}+\eta\right\rangle-\left\langle x^{\prime}+y^{\prime}, \eta^{\prime}\right\rangle\right)} a\left(x^{\prime}+y^{\prime}, \xi^{\prime}+\eta\right) \widehat{\varphi}\left(\xi^{\prime}\right) \overline{\varphi\left(x^{\prime}\right)} d x^{\prime} d \xi^{\prime} \\
= & (2 \pi)^{-2 d} e^{i\left(\left\langle y^{\prime}, \eta\right\rangle-\left\langle y^{\prime}, \eta^{\prime}\right\rangle\right)} \\
& \cdot \int_{\mathbb{R}^{2 d}} e^{i\left(\left\langle x^{\prime}, \xi^{\prime}\right\rangle+\left\langle x^{\prime}, \eta\right\rangle+\left\langle y^{\prime}, \xi^{\prime}\right\rangle-\left\langle y, \xi^{\prime}\right\rangle-\left\langle x^{\prime}, \eta^{\prime}\right\rangle\right)} a\left(x^{\prime}+y^{\prime}, \xi^{\prime}+\eta\right) \widehat{\varphi}\left(\xi^{\prime}\right) \overline{\varphi\left(x^{\prime}\right)} d x^{\prime} d \xi^{\prime},
\end{aligned}
$$

and hence, setting $x=x^{\prime}$ and $\xi=\xi^{\prime}$ :

$$
\left|K\left(z^{\prime}, z\right)\right|=(2 \pi)^{-2 d}\left|\int_{\mathbb{R}^{2 d}} e^{i\left(\left\langle x, \eta-\eta^{\prime}+\xi\right\rangle+\left\langle\xi, y^{\prime}-y\right\rangle\right)} a\left(x+y^{\prime}, \xi+\eta\right) \widehat{\varphi}(\xi) \overline{\varphi(x)} d x d \xi\right| .
$$

Writing, for $M, N \in \mathbb{N}_{0}$,

$$
\begin{aligned}
e^{i\left(\left\langle x, \eta-\eta^{\prime}+\xi\right\rangle+\left\langle\xi, y^{\prime}-y\right\rangle\right)} & =\left\langle\eta-\eta^{\prime}+\xi\right\rangle^{-2 M}\left(1-\Delta_{x}\right)^{M} e^{i\left(\left\langle x, \eta-\eta^{\prime}+\xi\right\rangle+\left\langle\xi, y^{\prime}-y\right\rangle\right)} \\
& =\left\langle y-y^{\prime}\right\rangle^{-2 N}\left\langle\eta-\eta^{\prime}+\xi\right\rangle^{-2 M}\left(1-\Delta_{x}\right)^{M} e^{i\left\langle x, \eta-\eta^{\prime}+\xi\right\rangle}\left(1-\Delta_{\xi}\right)^{N} e^{i\left\langle\xi, y^{\prime}-y\right\rangle}
\end{aligned}
$$

and integrating by parts in (4.13), we have

$$
\left|K\left(z^{\prime}, z\right)\right|=(2 \pi)^{-2 d}\left\langle y-y^{\prime}\right\rangle^{-2 N}\left|\int_{\mathbb{R}^{2 d}} e^{i\left(\left\langle x, \eta-\eta^{\prime}\right\rangle+\left\langle\xi, y^{\prime}-y\right\rangle\right)} \lambda_{N, M}\left(y^{\prime}, \eta^{\prime}, \eta, x, \xi\right) d x d \xi\right|
$$

where

$$
\begin{aligned}
& \lambda_{N, M}\left(y^{\prime}, \eta^{\prime}, \eta, x, \xi\right) \\
& =\left(1-\Delta_{\xi}\right)^{N}\left[e^{i\langle x, \xi\rangle}\left\langle\eta-\eta^{\prime}+\xi\right\rangle^{-2 M}\left(1-\Delta_{x}\right)^{M}\left(a\left(x+y^{\prime}, \xi+\eta\right) \widehat{\varphi}(\xi) \overline{\varphi(x)}\right)\right] .
\end{aligned}
$$

For $a \in S_{\omega}^{m}$, since $\varphi, \widehat{\varphi} \in \mathcal{S}_{\omega}\left(\mathbb{R}^{d}\right)$, we use the definition of symbol (Definition 4.2) and the seminorms (2.3) to obtain that for each $\lambda, \mu, \lambda^{\prime}, \mu^{\prime}, \lambda^{\prime \prime}, \mu^{\prime \prime}>0$ there is a positive constant 
$C:=C_{\lambda, \mu, \lambda^{\prime}, \mu^{\prime}, \lambda^{\prime \prime}, \mu^{\prime \prime}}$ such that for every $M, N \in \mathbb{N}_{0}$ :

$$
\begin{aligned}
\left|\lambda_{N, M}\left(y^{\prime}, \eta^{\prime}, \eta, x, \xi\right)\right| \leq C & \sum_{\gamma_{1}+\gamma_{2}+\gamma_{3}+\gamma_{4}=2 N} \frac{(2 N) !}{\gamma_{1} ! \gamma_{2} ! \gamma_{3} ! \gamma_{4} !} \sum_{\sigma_{1}+\sigma_{2}=2 M} \frac{(2 M) !}{\sigma_{1} ! \sigma_{2} !}\langle x\rangle^{\left|\gamma_{1}\right|}\left\langle\eta-\eta^{\prime}+\xi\right\rangle^{-2 M-\left|\gamma_{2}\right|} \\
& \times e^{\lambda \varphi_{\omega}^{*}\left(\frac{\left|\gamma_{3}\right|}{\lambda}\right)} e^{\mu \varphi_{\omega}^{*}\left(\frac{\left|\sigma_{1}\right|}{\mu}\right)} e^{m \omega(\xi+\eta)} e^{\lambda^{\prime} \varphi_{\omega}^{*}\left(\frac{\left|\gamma_{4}\right|}{\lambda^{\prime}}\right)} e^{-\mu^{\prime} \omega(\xi)} \\
& \times e^{\lambda^{\prime \prime} \varphi_{\omega}^{*}\left(\frac{\left|\sigma_{2}\right|}{\lambda^{\prime \prime}}\right)} e^{-\mu^{\prime \prime} \omega(x)} .
\end{aligned}
$$

We observe that

$$
\left\langle\eta-\eta^{\prime}+\xi\right\rangle^{-1} \leq \sqrt{2}\left\langle\eta-\eta^{\prime}\right\rangle^{-1}\langle\xi\rangle
$$

and, hence,

$$
\left\langle\eta-\eta^{\prime}+\xi\right\rangle^{-2 M-\left|\gamma_{2}\right|} \leq 2^{\left(2 M+\left|\gamma_{2}\right|\right) / 2}\left\langle\eta-\eta^{\prime}\right\rangle^{-2 M-\left|\gamma_{2}\right|}\langle\xi\rangle^{2 M+\left|\gamma_{2}\right|} .
$$

By (2.12) and by the subadditivity of $\omega$, for all $\tilde{\lambda}>0$ :

$$
\langle x\rangle^{\left|\gamma_{1}\right|} \leq e^{\tilde{\lambda} \varphi_{\omega}^{*}\left(\frac{\left|\gamma_{1}\right|}{\bar{\lambda}}\right)} e^{\tilde{\lambda} \omega(\langle x\rangle)} \leq e^{\tilde{\lambda} \varphi_{\omega}^{*}\left(\frac{\left|\gamma_{\tilde{\lambda}}\right|}{\bar{\lambda}}\right)} e^{\tilde{\lambda} \omega(1+|x|)} \leq C_{\tilde{\lambda}} e^{\tilde{\lambda} \varphi_{\omega}^{*}\left(\frac{\left|\gamma_{1}\right|}{\tilde{\lambda}}\right)} e^{\tilde{\lambda} \omega(x)}
$$

for $C_{\tilde{\lambda}}=e^{\tilde{\lambda} \omega(1)}$.

Analogously

$$
\langle\xi\rangle^{\left|\gamma_{2}\right|} \leq C_{\tilde{\lambda}} e^{\tilde{\lambda} \varphi_{\omega}^{*}\left(\frac{\left|\gamma_{2}\right|}{\tilde{\lambda}}\right)} e^{\tilde{\lambda} \omega(\xi)}
$$

Substituting (4.17) and (4.18) into (4.15), choosing $\mu^{\prime}=\tilde{\lambda}+m+1, \mu^{\prime \prime}=\tilde{\lambda}+1, \mu=\lambda=$ $\lambda^{\prime}=\lambda^{\prime \prime}=\tilde{\lambda}$ and applying (2.15) we obtain a constant $C_{\lambda}>0$ such that for all $M, N \in \mathbb{N}_{0}$ :

$$
\begin{aligned}
& \left|\lambda_{N, M}\left(y^{\prime}, \eta^{\prime}, \eta, x, \xi\right)\right| \leq C_{\lambda}(d \sqrt{2})^{2 M+2 N} \sum_{\gamma_{1}+\gamma_{2}+\gamma_{3}+\gamma_{4}=2 N} \frac{(2 N) !}{\gamma_{1} ! \gamma_{2} ! \gamma_{3} ! \gamma_{4} !} d^{-2 N} \sum_{\sigma_{1}+\sigma_{2}=2 M} \frac{(2 M) !}{\sigma_{1} ! \sigma_{2} !} d^{-2 M} \\
& \times e^{\lambda \varphi_{\omega}^{*}\left(\frac{2 N}{\lambda}\right)} e^{\lambda \varphi_{\omega}^{*}\left(\frac{2 M}{\lambda}\right)}\left\langle\eta-\eta^{\prime}\right\rangle^{-2 M-\left|\gamma_{2}\right|}\langle\xi\rangle^{2 M} e^{-\omega(\xi)-\omega(x)} e^{m \omega\left(\eta-\eta^{\prime}\right)} e^{m \omega\left(\eta^{\prime}\right)} \\
& \leq C_{\lambda}(d \sqrt{2})^{2 M+2 N} e^{\lambda \varphi_{\omega}^{*}\left(\frac{2 N}{\lambda}\right)} e^{\lambda \varphi_{\omega}^{*}\left(\frac{2 M}{\lambda}\right)} \\
& \times\left\langle\eta-\eta^{\prime}\right\rangle^{-2 M}\langle\xi\rangle^{2 M} e^{-\omega(\xi)-\omega(x)} e^{m \omega\left(\eta-\eta^{\prime}\right)} e^{m \omega\left(\eta^{\prime}\right)} .
\end{aligned}
$$

By [8, Lemma 4.5] we have that for every $\lambda^{\prime}>0$ there exists $C_{\lambda^{\prime}}>0$ such that

$$
\left|\lambda_{N, M}\left(y^{\prime}, \eta^{\prime}, \eta, x, \xi\right)\right| \leq C_{\lambda^{\prime}} e^{\lambda^{\prime} \varphi_{\omega}^{*}\left(\frac{2 N}{\lambda^{\prime}}\right)} e^{\lambda^{\prime} \varphi_{\omega}^{*}\left(\frac{2 M}{\lambda^{\prime}}\right)}\left\langle\eta-\eta^{\prime}\right\rangle^{-2 M}\langle\xi\rangle^{2 M} e^{-\omega(\xi)-\omega(x)} e^{m \omega\left(\eta-\eta^{\prime}\right)} e^{m \omega\left(\eta^{\prime}\right)} .
$$

Now, we turn to formula (4.14) and we have that for all $\lambda$ there is a constant $C_{\lambda}>0$ such that for every $M, N \in \mathbb{N}_{0}$ :

$$
\begin{aligned}
\left|K\left(z^{\prime}, z\right)\right| \leq & C_{\lambda}\left\langle y-y^{\prime}\right\rangle^{-2 N} e^{\lambda \varphi_{\omega}^{*}\left(\frac{2 N}{\lambda}\right)}\left\langle\eta-\eta^{\prime}\right\rangle^{-2 M} e^{\lambda \varphi_{\omega}^{*}\left(\frac{2 M}{\lambda}\right)} e^{m \omega\left(\eta-\eta^{\prime}\right)} e^{m \omega\left(\eta^{\prime}\right)} \\
& \times\left(\int_{\mathbb{R}^{d}} e^{-\omega(x)} d x\right)\left(\int_{\mathbb{R}^{d}}\langle\xi\rangle^{2 M} e^{-\omega(\xi)} d \xi\right) .
\end{aligned}
$$

We observe that the integrals are convergent (the second one, for all $M \in \mathbb{N}_{0}$ ) by Lemma 4.1. We take the infimum on $N$ and $M$ separately and use the property that the infimum of the pointwise product of two sets of positive numbers is the product of the infimums of the two sets. Therefore, we apply (4.10) for $\sigma=2$ to obtain (possibly) a new constant $C_{\lambda}^{\prime}$ such that:

$$
\left|K\left(z^{\prime}, z\right)\right| \leq C_{\lambda}^{\prime} e^{-\left(\lambda-\frac{2}{b}\right) \omega\left(y-y^{\prime}\right)} e^{-\left(\lambda-\frac{2}{b}\right) \omega\left(\eta-\eta^{\prime}\right)} e^{m \omega\left(\eta-\eta^{\prime}\right)} e^{m \omega\left(\eta^{\prime}\right)},
$$


which proves (4.11) by the arbitrariness of $\lambda$.

Now, we want to prove (4.12). To do so, we apply (4.16) only to $\left\langle\eta-\eta^{\prime}+\xi\right\rangle^{-M}$ in (4.15) and, by the same computations to get (4.19), we have that if $a(z)=0$ for $z \in \Gamma \backslash \overline{B(0, R)}$, for every $\lambda>0$ there is a constant $C_{\lambda}>0$ such that

$$
\begin{aligned}
\left|K\left(z^{\prime}, z\right)\right| \leq C_{\lambda} & \left\langle y-y^{\prime}\right\rangle^{-2 N} e^{\lambda \varphi^{*}\left(\frac{2 N}{\lambda}\right)}\left\langle\eta-\eta^{\prime}\right\rangle^{-M} e^{\lambda \varphi_{\omega}^{*}\left(\frac{2 M}{\lambda}\right)} e^{m \omega\left(\eta-\eta^{\prime}\right)} e^{m \omega\left(\eta^{\prime}\right)} \\
& \times \int_{D_{y^{\prime}, \eta}}\left\langle\eta^{\prime}-(\xi+\eta)\right\rangle^{-M} e^{-\omega(x)}\langle\xi\rangle^{M} e^{-\omega(\xi)} d x d \xi
\end{aligned}
$$

where

$$
D_{y^{\prime}, \eta}:=\left\{(x, \xi) \in \mathbb{R}^{2 d}:\left(x+y^{\prime}, \xi+\eta\right) \in\left(\mathbb{R}^{2 d} \backslash \Gamma\right) \cup \overline{B(0, R)}\right\} .
$$

We now want to estimate (4.21) for $z^{\prime}=\left(y^{\prime}, \eta^{\prime}\right) \in \Gamma^{\prime}$ and $z=(y, \eta) \in \mathbb{R}^{2 d}$. By [35, pg 643] we know that

$$
\left\langle y^{\prime}\right\rangle\left\langle\eta^{\prime}\right\rangle \leq C\langle x\rangle^{2}\left\langle\eta^{\prime}-(\xi+\eta)\right\rangle^{2}, \quad z^{\prime} \in \Gamma^{\prime} \backslash B(0,2 R), z \in \mathbb{R}^{2 d},(x, \xi) \in D_{y^{\prime}, \eta},
$$

for some constant $C>0$.

We plug (4.22) into (4.21) and apply [5, Prop. 2.1(g)] to obtain, for $z^{\prime} \in \Gamma^{\prime} \backslash B(0,2 R)$ and $z \in \mathbb{R}^{2 d}$,

$$
\begin{aligned}
\left|K\left(z^{\prime}, z\right)\right| \leq & C^{M / 2} C_{\lambda}\left\langle y-y^{\prime}\right\rangle^{-2 N} e^{\lambda \varphi_{\omega}^{*}\left(\frac{2 N}{\lambda}\right)} \\
& \times\left\langle\eta-\eta^{\prime}\right\rangle^{-M} e^{\frac{\lambda}{2} \varphi_{\omega}^{*}\left(\frac{M}{\lambda / 2}\right)} e^{\frac{\lambda}{4} \varphi_{\omega}^{*}\left(\frac{M / 2}{\lambda / 4}\right)}\left\langle y^{\prime}\right\rangle^{-M / 2} e^{\frac{\lambda}{4} \varphi_{\omega}^{*}\left(\frac{M / 2}{\lambda / 4}\right)}\left\langle\eta^{\prime}\right\rangle^{-M / 2} \\
& \times e^{m \omega\left(\eta-\eta^{\prime}\right)} e^{m \omega\left(\eta^{\prime}\right)} \int_{D_{y^{\prime}, \eta}}\langle x\rangle^{M}\langle\xi\rangle^{M} e^{-\omega(x)} e^{-\omega(\xi)} d x d \xi .
\end{aligned}
$$

Proceeding as in the case before (taking the infimum in $M$ and $N$ separately), we obtain, from (4.10), that for every $\lambda>0$ there exists another constant $C_{\lambda}>0$ such that

$$
\begin{aligned}
\left|K\left(z^{\prime}, z\right)\right| \leq & C_{\lambda} e^{-\left(\lambda-\frac{2}{b}\right) \omega\left(y-y^{\prime}\right)} e^{-\left(\frac{\lambda}{2}-\frac{1}{b}\right) \omega\left(\eta-\eta^{\prime}\right)} \\
& \times e^{-\left(\frac{\lambda}{4}-\frac{1}{2 b}\right) \omega\left(y^{\prime}\right)} e^{-\left(\frac{\lambda}{4}-\frac{1}{2 b}\right) \omega\left(\eta^{\prime}\right)} e^{m \omega\left(\eta-\eta^{\prime}\right)} e^{m \omega\left(\eta^{\prime}\right)} \\
\leq & C_{\lambda} e^{-\bar{\lambda} \omega\left(y-y^{\prime}\right)} e^{-\bar{\lambda} \omega\left(\eta-\eta^{\prime}\right)} e^{-2 \bar{\lambda} \omega\left(y^{\prime}\right)} e^{-2 \bar{\lambda} \omega\left(\eta^{\prime}\right)}
\end{aligned}
$$

for $\bar{\lambda}=\frac{\lambda}{8}-\frac{2}{b}-m$ and $z^{\prime} \in \Gamma^{\prime} \backslash B(0,2 R), z \in \mathbb{R}^{2 d}$. The estimate (4.12) for $\left|z^{\prime}\right| \leq 2 R$ follows from the case before, so the proof is complete.

Remark 4.5. For $a \in S_{\omega}^{m}, m \in \mathbb{R}$, and $K \in C^{\infty}\left(\mathbb{R}^{4 d}\right)$ defined by (4.7) the integral in (4.6) is well defined also for $u \in \mathcal{S}_{\omega}^{\prime}\left(\mathbb{R}^{d}\right)$. In fact, (2.9) and (4.11) imply that there exist $\tilde{C}, \tilde{\lambda}>0$ and that for every $\lambda>0$ there exists $C_{\lambda}>0$ such that

$$
\begin{aligned}
\left|K\left(z^{\prime}, z\right) V_{\varphi} u(z)\right| & \leq \tilde{C} C_{\lambda} e^{-\lambda \omega\left(y-y^{\prime}\right)+(m-\lambda) \omega\left(\eta-\eta^{\prime}\right)} e^{m \omega\left(\eta^{\prime}\right)} e^{\tilde{\lambda} \omega(y)+\tilde{\lambda} \omega(\eta)} \\
& \leq \tilde{C} C_{\lambda} e^{\lambda \omega\left(y^{\prime}\right)+(m+\lambda) \omega\left(\eta^{\prime}\right)} e^{(\tilde{\lambda}-\lambda) \omega(y)+(m+\tilde{\lambda}-\lambda) \omega(\eta)} \in L^{1}\left(\mathbb{R}_{z=(y, \eta)}^{2 d}\right)
\end{aligned}
$$

if $\lambda>\max \{\tilde{\lambda}, m+\tilde{\lambda}\}$, by $(4.2)$.

We now want to extend Lemma 4.3 for $u \in \mathcal{S}_{\omega}^{\prime}\left(\mathbb{R}^{d}\right)$. To this aim we first need the next two results.

Proposition 4.6. The space $\mathcal{S}_{\omega}\left(\mathbb{R}^{d}\right)$ is dense in $\mathcal{S}_{\omega}^{\prime}\left(\mathbb{R}^{d}\right)$. 
Proof. Let us consider the inclusion

$$
\begin{aligned}
i: \mathcal{S}_{\omega}\left(\mathbb{R}^{d}\right) & \hookrightarrow \mathcal{S}_{\omega}^{\prime}\left(\mathbb{R}^{d}\right) \\
f & \mapsto\langle i(f), \varphi\rangle:=\int_{\mathbb{R}^{d}} f(x) \overline{\varphi(x)} d x \quad \forall \varphi \in \mathcal{S}_{\omega}\left(\mathbb{R}^{d}\right) .
\end{aligned}
$$

To show that the image is dense we take $T \in\left(\mathcal{S}_{\omega}^{\prime}\left(\mathbb{R}^{d}\right)\right)^{\prime}$ such that $\left.T\right|_{\mathcal{S}_{\omega}}=0$ and prove that $T \equiv 0$ (Hahn-Banach theorem for locally convex spaces).

Since $\mathcal{S}_{\omega}\left(\mathbb{R}^{d}\right)$ is reflexive, there exists a unique $f \in \mathcal{S}_{\omega}\left(\mathbb{R}^{d}\right)$ such that

$$
T(\varphi)=\int_{\mathbb{R}^{d}} f(x) \overline{\varphi(x)} d x=0, \quad \forall \varphi \in \mathcal{S}_{\omega}\left(\mathbb{R}^{d}\right)
$$

because of $\left.T\right|_{\mathcal{S}_{\omega}}=0$. Therefore $f=0$, i.e. $T \equiv 0$.

Proposition 4.7. Let $\varphi \in \mathcal{S}_{\omega}\left(\mathbb{R}^{d}\right) \backslash\{0\}$. Then

$$
V_{\varphi}: \mathcal{S}_{\omega}^{\prime}\left(\mathbb{R}^{d}\right) \longrightarrow \mathcal{S}_{\omega}^{\prime}\left(\mathbb{R}^{2 d}\right)
$$

is continuous.

Proof. We already know that

$$
V_{\varphi}^{*}: \mathcal{S}_{\omega}\left(\mathbb{R}^{2 d}\right) \longrightarrow \mathcal{S}_{\omega}\left(\mathbb{R}^{d}\right)
$$

is continuous by (2.21). It follows that

$$
\left(V_{\varphi}^{*}\right)^{*}: \mathcal{S}_{\omega}^{\prime}\left(\mathbb{R}^{d}\right) \longrightarrow \mathcal{S}_{\omega}^{\prime}\left(\mathbb{R}^{2 d}\right)
$$

is continuous and moreover $\left.\left(V_{\varphi}^{*}\right)^{*}\right|_{\mathcal{S}_{\omega}\left(\mathbb{R}^{d}\right)}=V_{\varphi}$ because, for $f, g \in \mathcal{S}_{\omega}\left(\mathbb{R}^{d}\right)$,

$$
\left\langle\left(V_{\varphi}^{*}\right)^{*} f, g\right\rangle=\left\langle f, V_{\varphi}^{*} g\right\rangle=\left\langle V_{\varphi} f, g\right\rangle .
$$

Since $\mathcal{S}_{\omega}\left(\mathbb{R}^{d}\right)$ is dense in $\mathcal{S}_{\omega}^{\prime}\left(\mathbb{R}^{d}\right)$ by Proposition 4.6 , we have that $\left(V_{\varphi}^{*}\right)^{*}$ is the continuous extension of $V_{\varphi}$ to $\mathcal{S}_{\omega}^{\prime}\left(\mathbb{R}^{d}\right)$ and, hence, $V_{\varphi}$ is continuous on $\mathcal{S}_{\omega}^{\prime}\left(\mathbb{R}^{d}\right)$ also.

Now, we need amplitudes $a(x, y, \xi)$, instead of symbols $a(x, \xi)$.

Definition 4.8. Given $m \in \mathbb{R}$, we say that $a(x, y, \xi) \in C^{\infty}\left(\mathbb{R}^{3 d}\right)$ is an amplitude in the space $S_{\omega}^{m}$ if for every $\lambda, \mu>0$ there is $C_{\lambda, \mu}>0$ such that

$$
\left|\partial_{x}^{\alpha} \partial_{y}^{\gamma} \partial_{\xi}^{\beta} a(x, y, \xi)\right| \leq C_{\lambda, \mu} e^{\lambda \varphi^{*}\left(\frac{|\alpha+\gamma|}{\lambda}\right)+\mu \varphi^{*}\left(\frac{|\beta|}{\mu}\right)} e^{m \omega(\xi)},
$$

for all $(x, y, \xi) \in \mathbb{R}^{3 d}$ and $\alpha, \beta, \gamma \in \mathbb{N}_{0}^{d}$.

Now, proceeding in a similar way to that of Proposition 1.9 and Theorem 2.2 of [15], one can prove that if $a(x, y, \xi) \in S_{\omega}^{m}$ is an amplitude as in Definition 4.8, the operator acting on $\mathcal{S}_{\omega}$, given by the iterated integral

$$
A(f)(x):=\int_{\mathbb{R}^{d}}\left(\int_{\mathbb{R}^{d}} e^{i\langle x-y, \xi\rangle} a(x, y, \xi) f(y) d y\right) d \xi, \quad f \in \mathcal{S}_{\omega},
$$

is well defined and continuous from $\mathcal{S}_{\omega}$ into itself. The operator $A$ is called pseudo-differential operator of type $\omega$ with amplitude $a(x, y, \xi)$. Moreover, $A$ can be extended continuously to 
the dual space $\tilde{A}: \mathcal{S}_{\omega}^{\prime} \rightarrow \mathcal{S}_{\omega}^{\prime}$ in a standard way (see [15, Theorem 2.5]). In particular, the Kohn-Nirenberg quantization defined in (4.3) is a pseudo-differential operator with amplitude

$$
a(x, y, \xi):=(2 \pi)^{-d} p(x, \xi),
$$

where $p(x, \xi)$ is a symbol as in Definition 4.2 .

As a consequence of the above considerations and of the estimates of the kernel in Proposition 4.4, we obtain the following result:

Corollary 4.9. Let $a(x, \xi) \in S_{\omega}^{m}$ a symbol as in Definition 4.2, $\varphi \in \mathcal{S}_{\omega}\left(\mathbb{R}^{d}\right)$ with $\|\varphi\|_{L^{2}}=1$ and $u \in \mathcal{S}_{\omega}^{\prime}\left(\mathbb{R}^{d}\right)$. Then, for $K\left(z^{\prime}, z\right)$ as in $(4.7)$, we have

$$
V_{\varphi} a(x, D) u\left(z^{\prime}\right)=\int_{\mathbb{R}^{2 d}} K\left(z^{\prime}, z\right) V_{\varphi} u(z) d z,
$$

for all $z^{\prime} \in \mathbb{R}^{2 d}$.

Proof. Since $V_{\varphi}$ operates on $\mathcal{S}_{\omega}^{\prime}$, from the previous comments it is clear that $V_{\varphi} a(x, D)$ can be extended to $\mathcal{S}_{\omega}^{\prime}\left(\mathbb{R}^{d}\right)$. We take $u \in \mathcal{S}_{\omega}^{\prime}\left(\mathbb{R}^{d}\right)$. By Proposition 4.6, there exists a sequence $\left\{u_{n}\right\}_{n \in \mathbb{N}} \subset \mathcal{S}_{\omega}\left(\mathbb{R}^{d}\right)$ which converges to $u$ in $\mathcal{S}_{\omega}^{\prime}$ and, hence,

$$
\int_{\mathbb{R}^{2 d}} K\left(z^{\prime}, z\right) V_{\varphi} u_{n}(z) d z=V_{\varphi} a(x, D) u_{n}\left(z^{\prime}\right) \longrightarrow V_{\varphi} a(x, D) u\left(z^{\prime}\right) \quad \text { in } \mathcal{S}_{\omega}^{\prime}\left(\mathbb{R}^{2 d}\right)
$$

We want to prove that

$$
\int_{\mathbb{R}^{2 d}} K\left(z^{\prime}, z\right) V_{\varphi} u_{n}(z) d z \longrightarrow \int_{\mathbb{R}^{2 d}} K\left(z^{\prime}, z\right) V_{\varphi} u(z) d z
$$

using Lebesgue's dominated convergence theorem. First, it is easy to see that $\left\{V_{\varphi} u_{n}(z)\right\}_{n \in \mathbb{N}}$ converges pointwise to $V_{\varphi} u(z)$ for every $z \in \mathbb{R}^{2 d}$ from the definition of the short-time Fourier transform.

Now, since $\left\{u_{n}\right\}_{n \in \mathbb{N}}$ is bounded in $\mathcal{S}_{\omega}^{\prime}\left(\mathbb{R}^{d}\right)$, it is equicontinuous there. So, there exist a constant $C>0$ and a seminorm $q$ on $\mathcal{S}_{\omega}\left(\mathbb{R}^{d}\right)$ such that

$$
\left|\left\langle u_{n}, \varphi\right\rangle\right| \leq C q(\varphi), \quad \varphi \in \mathcal{S}_{\omega}\left(\mathbb{R}^{d}\right)
$$

This yields a uniform estimate of the inequality (2.9) (see the proof of [19, Theorem 2.4]) in the sense:

$$
\left|V_{\varphi} u_{n}(z)\right| \leq \tilde{C} e^{\tilde{\lambda} \omega(z)}, \quad z \in \mathbb{R}^{2 d}, n \in \mathbb{N}
$$

for some $\tilde{C}, \tilde{\lambda}>0$ independent of $n$ and $z$. From (4.27) and (4.23) we have that $K\left(z^{\prime}, z\right) V_{\varphi} u_{n}(z)$ is dominated by a function in $L^{1}\left(\mathbb{R}_{z}^{2 d}\right)$.

Therefore (4.26) is satisfied and hence, from (4.25),

$$
V_{\varphi} a(x, D) u\left(z^{\prime}\right)=\int_{\mathbb{R}^{2 d}} K\left(z^{\prime}, z\right) V_{\varphi} u(z) d z
$$

also for $u \in \mathcal{S}_{\omega}^{\prime}\left(\mathbb{R}^{d}\right)$.

We recall the notion of conic support from [35]: 
Definition 4.10. For $a \in \mathcal{D}^{\prime}\left(\mathbb{R}^{2 d}\right)$ the conic support of $a$, denoted by cone $\operatorname{supp}(a)$, is the set of all $z \in \mathbb{R}^{2 d} \backslash\{0\}$ such that any open conic set $\Gamma \subset \mathbb{R}^{2 d} \backslash\{0\}$ containing z satisfies that

$$
\overline{\operatorname{supp}(a) \cap \Gamma} \text { is not compact in } \mathbb{R}^{2 d} \text {. }
$$

We have the following

Proposition 4.11. If $m \in \mathbb{R}, a \in S_{\omega}^{m}$ and $u \in \mathcal{S}_{\omega}^{\prime}\left(\mathbb{R}^{d}\right)$, then

$$
\mathrm{WF}_{\omega}^{\prime}(a(x, D) u) \subseteq \text { conesupp }(a) .
$$

Proof. Let $0 \neq z_{0} \notin$ cone $\operatorname{supp}(a)$. This means that there exists an open conic set $\Gamma \subset \mathbb{R}^{2 d} \backslash\{0\}$ containing $z_{0}$ and such that $a(z)=0$ for $z \in \Gamma \backslash \overline{B(0, R)}$ for some $R>0$. Then, from Proposition 4.4, for every open conic set $\Gamma^{\prime} \subseteq \mathbb{R}^{2 d} \backslash\{0\}$ with $\overline{\Gamma^{\prime} \cap S_{2 d-1}} \subseteq \Gamma$ we have that the kernel $K\left(z^{\prime}, z\right)$ defined by (4.7) satisfies the estimate (4.12) for all $z^{\prime} \in \Gamma^{\prime}$ and $z \in \mathbb{R}^{2 d}$.

We argue as in Corollary 4.9 and use (4.12) to obtain that formula (4.24) holds for all $z^{\prime} \in \Gamma^{\prime}$ and therefore there exist $C, \bar{\lambda}>0$, and for every $\lambda, N>0$ there exists $C_{\lambda, N}>0$ such that, for all $z^{\prime} \in \Gamma^{\prime}$,

$$
\begin{aligned}
\left|V_{\varphi}(a(x, D) u)\left(z^{\prime}\right)\right| \leq & \int_{\mathbb{R}^{2 d}}\left|K\left(z^{\prime}, z\right)\right| \cdot\left|V_{\varphi} u(z)\right| d z \\
\leq & C_{\lambda, N} e^{-2(\lambda+N) \omega\left(y^{\prime}\right)} e^{-2(\lambda+N) \omega\left(\eta^{\prime}\right)} \\
& \cdot \int_{\mathbb{R}^{2 d}} e^{-(\lambda+N) \omega\left(y-y^{\prime}\right)} e^{-(\lambda+N) \omega\left(\eta-\eta^{\prime}\right)}\left|V_{\varphi} u(y, \eta)\right| d y d \eta \\
\leq & C C_{\lambda, N} e^{-2(\lambda+N) \omega\left(y^{\prime}\right)} e^{-2(\lambda+N) \omega\left(\eta^{\prime}\right)} \\
& \cdot \int_{\mathbb{R}^{2 d}} e^{-(\lambda+N) \omega\left(y-y^{\prime}\right)} e^{-(\lambda+N) \omega\left(\eta-\eta^{\prime}\right)} e^{\bar{\lambda} \omega(y, \eta)} d y d \eta .
\end{aligned}
$$

It follows, by the subadditivity of $\omega$, that

$$
\begin{aligned}
\left|V_{\varphi} a(x, D) u\left(z^{\prime}\right)\right| \leq & C C_{\lambda, N} e^{-2(\lambda+N) \omega\left(y^{\prime}\right)} e^{-2(\lambda+N) \omega\left(\eta^{\prime}\right)} \\
& \cdot \int_{\mathbb{R}^{2 d}} e^{-(\lambda+N) \omega(y)+(\lambda+N) \omega\left(y^{\prime}\right)} e^{-(\lambda+N) \omega(\eta)+(\lambda+N) \omega\left(\eta^{\prime}\right)} e^{\bar{\lambda} \omega(y)+\bar{\lambda} \omega(\eta)} d y d \eta \\
\leq & C C_{\lambda, N} e^{-\lambda \omega\left(y^{\prime}\right)} e^{-\lambda \omega\left(\eta^{\prime}\right)} \int_{\mathbb{R}^{2 d}} e^{(\bar{\lambda}-N) \omega(y)} e^{(\bar{\lambda}-N) \omega(\eta)} d y d \eta \\
\leq & C_{\lambda} e^{-\lambda \omega\left(y^{\prime}\right)} e^{-\lambda \omega\left(\eta^{\prime}\right)} \leq C_{\lambda} e^{-\lambda \omega\left(z^{\prime}\right)} \quad \forall z^{\prime}=\left(y^{\prime}, \eta^{\prime}\right) \in \Gamma^{\prime}
\end{aligned}
$$

for some $C_{\lambda}>0$ if we choose $N$ sufficiently large so that the integral in (4.28) converges.

This proves that $z_{0} \notin \mathrm{WF}_{\omega}^{\prime}(a(x, D) u)$ by Definition 3.1, and the proof is complete.

Since our weight functions are non-quasianalytic, we can obtain the following consequence of Proposition 4.11.

Corollary 4.12. Let $a \in \mathcal{S}_{\omega}\left(\mathbb{R}^{2 d}\right)$ with compact support, and consider the corresponding pseudodifferential operator $a(x, D)$, cf. (4.3). Then $a(x, D)$ is globally $\omega$-regularizing, in the sense that for every $u \in \mathcal{S}_{\omega}^{\prime}\left(\mathbb{R}^{d}\right)$ we have $a(x, D) u \in \mathcal{S}_{\omega}\left(\mathbb{R}^{d}\right)$.

Proof. It is easy to see that $a \in S_{\omega}^{0}$. Consequently, the corresponding pseudo-differential operator $a(x, D)$ can be extended to $\mathcal{S}_{\omega}^{\prime}\left(\mathbb{R}^{d}\right)$. Since the support of $a$ is compact, we have that 
cone $\operatorname{supp}(a)=\emptyset$. From Proposition 4.11 we get $\mathrm{WF}_{\omega}^{\prime}(a(x, D) u)=\emptyset$. We apply Proposition 3.18 to conclude.

In the next part of the section we consider other kind of operators, proving that their application to ultradistributions does not enlarge the wave front set. We start from the operators with polynomial coefficients.

Theorem 4.13. Let $m>0$ be an integer, and consider

$$
A(x, D)=\sum_{|\alpha+\beta| \leq m} c_{\alpha \beta} x^{\alpha} D_{x}^{\beta},
$$

where $c_{\alpha \beta} \in \mathbb{C}$. Then for every $u \in \mathcal{S}_{\omega}^{\prime}\left(\mathbb{R}^{d}\right)$ we have

$$
\mathrm{WF}_{\omega}^{\prime}(A(x, D) u) \subseteq \mathrm{WF}_{\omega}^{\prime}(u)
$$

Proof. We fix a window function $\varphi \in \mathcal{S}_{\omega}\left(\mathbb{R}^{d}\right)$, and, for $\nu \in \mathbb{N}_{0}^{d}$ we write $\varphi_{\nu}$ for the function

$$
\varphi_{\nu}(x)=x^{\nu} \varphi(x) \text {. }
$$

For every $\alpha \in \mathbb{N}_{0}^{d}$ and $z=(y, \eta) \in \mathbb{R}^{2 d}$ we obtain by induction on $|\alpha|$ that

$$
x^{\alpha} \Pi(z) \varphi=\sum_{\nu \leq \alpha}\left(\begin{array}{l}
\alpha \\
\nu
\end{array}\right) y^{\alpha-\nu} \Pi(z) \varphi_{\nu} .
$$

We have indeed that for $|\alpha|=1$, writing $\mathbf{1}_{j}$ for the multi-index in $\mathbb{N}_{0}^{d}$ having 1 in the $j$-th position and 0 elsewhere, we have

$$
x_{j} \Pi(z) \varphi=y_{j} \Pi(z) \varphi+\Pi(z) \varphi_{\mathbf{1}_{j}}
$$

we suppose now that (4.29) is true for every $|\alpha|=n$, and prove it for $\tilde{\alpha}$ with $|\tilde{\alpha}|=n+1$. There exists $j \in\{1, \ldots, d\}$ such that $\tilde{\alpha}=\alpha+\mathbf{1}_{j}$. Then by the inductive hypothesis we have

$$
\begin{aligned}
x^{\tilde{\alpha}} \Pi(z) \varphi & =x_{j} \sum_{\nu \leq \alpha}\left(\begin{array}{l}
\alpha \\
\nu
\end{array}\right) y^{\alpha-\nu} \Pi(z) \varphi_{\nu} \\
& =\sum_{\nu \leq \alpha}\left(\begin{array}{l}
\alpha \\
\nu
\end{array}\right)\left[y^{\alpha-\nu+\mathbf{1}_{j}} \Pi(z) \varphi_{\nu}+y^{\alpha-\nu} \Pi(z) \varphi_{\nu+\mathbf{1}_{j}}\right] \\
& =y^{\tilde{\alpha}} \Pi(z) \varphi+\Pi(z) \varphi_{\tilde{\alpha}}+\sum_{\substack{\nu \leq \alpha \\
\nu \neq 0}}\left[\left(\begin{array}{l}
\alpha \\
\nu
\end{array}\right)+\left(\begin{array}{c}
\alpha \\
\nu-\mathbf{1}_{j}
\end{array}\right)\right] y^{\tilde{\alpha}-\nu} \Pi(z) \varphi_{\nu} \\
& =\sum_{\nu \leq \tilde{\alpha}}\left(\begin{array}{l}
\tilde{\alpha} \\
\nu
\end{array}\right) y^{\tilde{\alpha}-\nu} \Pi(z) \varphi_{\nu},
\end{aligned}
$$

and so (4.29) is proved. From the definition of short-time Fourier transform we have

$$
V_{\varphi}\left(x^{\alpha} u\right)(z)=\left\langle x^{\alpha} u, \Pi(z) \varphi\right\rangle=\left\langle u, x^{\alpha} \Pi(z) \varphi\right\rangle
$$

and so by (4.29) we get

$$
V_{\varphi}\left(x^{\alpha} u\right)(z)=\sum_{\nu \leq \alpha}\left(\begin{array}{l}
\alpha \\
\nu
\end{array}\right) y^{\alpha-\nu} V_{\varphi_{\nu}} u(z)
$$


Concerning differentiation, since

$$
V_{\varphi}\left(D^{\beta} u\right)(z)=\left\langle D^{\beta} u, \Pi(z) \varphi\right\rangle=\left\langle u, D^{\beta}(\Pi(z) \varphi)\right\rangle
$$

a direct computation shows that

$$
V_{\varphi}\left(D^{\beta} u\right)(z)=\sum_{\mu \leq \beta}\left(\begin{array}{l}
\beta \\
\mu
\end{array}\right) \eta^{\beta-\mu} V_{D^{\mu} \varphi} u
$$

From (4.30) and (4.31) we finally obtain

$$
\begin{aligned}
V_{\varphi}(A(x, D) u)(y, \eta) & =\sum_{|\alpha+\beta| \leq m} c_{\alpha \beta} V_{\varphi}\left(x^{\alpha} D_{x}^{\beta} u\right)(y, \eta) \\
& =\sum_{|\alpha+\beta| \leq m} \sum_{\substack{\nu \leq \alpha \\
\mu \leq \beta}} c_{\alpha \beta}\left(\begin{array}{l}
\alpha \\
\nu
\end{array}\right)\left(\begin{array}{l}
\beta \\
\mu
\end{array}\right) y^{\alpha-\nu} \eta^{\beta-\mu} V_{D^{\mu} \varphi_{\nu}} u(y, \eta) .
\end{aligned}
$$

On the other hand, it is not difficult to see that for every $\mu, \nu \in \mathbb{N}_{0}^{d}, D^{\mu} \varphi_{\nu} \in \mathcal{S}_{\omega}\left(\mathbb{R}^{d}\right)$.

Suppose now that $z_{0}=\left(y_{0}, \eta_{0}\right) \notin \mathrm{WF}_{\omega}^{\prime}(u), z_{0} \in \mathbb{R}^{2 d} \backslash\{0\}$. Then, there exists an open conic set $\Gamma \subseteq \mathbb{R}^{2 d} \backslash\{0\}$ containing $z_{0}$ and such that

$$
\sup _{z \in \Gamma} e^{\lambda \omega(z)}\left|V_{\varphi} u(z)\right|<+\infty, \quad \lambda>0
$$

From Proposition 3.2 we have that for every $\mu, \nu \in \mathbb{N}_{0}^{d}$ and for every open conic set $\Gamma^{\prime} \subseteq \mathbb{R}^{2 d} \backslash\{0\}$ containing $z_{0}$ and such that $\overline{\Gamma^{\prime} \cap S_{2 d-1}} \subseteq \Gamma$,

$$
\sup _{z \in \Gamma^{\prime}} e^{\lambda \omega(z)}\left|V_{D^{\mu} \varphi_{\nu}} u(z)\right|<+\infty \quad \forall \lambda>0 .
$$

From (4.32), for every $k>0$ we get

$$
e^{\lambda \omega(z)}\left|V_{\varphi}(A(x, D) u)(z)\right| \leq \sum_{|\alpha+\beta| \leq m} \sum_{\substack{\nu \leq \alpha \\
\mu \leq \beta}} c_{\alpha \beta}\left(\begin{array}{l}
\alpha \\
\nu
\end{array}\right)\left(\begin{array}{l}
\beta \\
\mu
\end{array}\right) e^{-k \omega(z)}\left|y^{\alpha-\nu} \eta^{\beta-\mu}\right| e^{(\lambda+k) \omega(z)}\left|V_{D^{\mu} \varphi_{\nu}} u(z)\right| .
$$

Since $|\alpha-\nu|+|\beta-\mu| \leq m$, from (4.2) we have that for any $m \in \mathbb{N}, m \log (t) \leq \omega(t)$ for $t>0$ large enough. So, $t^{m} \leq e^{\omega(t)}$ for $t>0$ large enough, and hence

$$
\sup _{z \in \mathbb{R}^{2 d}} e^{-k \omega(z)}\left|y^{\alpha-\nu} \eta^{\beta-\mu}\right|<+\infty,
$$

for every $\nu \leq \alpha$ and $\mu \leq \beta$. Therefore, from (4.33) we obtain

$$
\sup _{z \in \Gamma^{\prime}} e^{\lambda \omega(z)}\left|V_{\varphi}(A(x, D) u)(z)\right|<+\infty, \quad \lambda>0
$$

which means that $z_{0} \notin \mathrm{WF}_{\omega}^{\prime}(A(x, D) u)$, and the proof is complete.

We now want to prove an analogue of Theorem 4.13 for the case of localization operators. We recall here the definition of such operators and prove some results that are needed for our purpose. Given two window functions $\psi, \gamma \in \mathcal{S}_{\omega}\left(\mathbb{R}^{d}\right) \backslash\{0\}$ and a symbol $a \in \mathcal{S}_{\omega}^{\prime}\left(\mathbb{R}^{2 d}\right)$, the corresponding localization operator $L_{\psi, \gamma}^{a}$ is defined, for $f \in \mathcal{S}_{\omega}\left(\mathbb{R}^{d}\right)$, as

$$
L_{\psi, \gamma}^{a} f=V_{\gamma}^{*}\left(a \cdot V_{\psi} f\right) .
$$


From Proposition 2.9 we have that

$$
L_{\psi, \gamma}^{a}: \mathcal{S}_{\omega}\left(\mathbb{R}^{d}\right) \rightarrow \mathcal{S}_{\omega}^{\prime}\left(\mathbb{R}^{d}\right)
$$

We want now to consider symbols in a smaller class than $\mathcal{S}_{\omega}^{\prime}\left(\mathbb{R}^{2 d}\right)$, in order to apply the corresponding localization operator to distributions. We have the following result.

Lemma 4.14. Let $a(z), z \in \mathbb{R}^{2 d}$, be a measurable function such that there exist $\tau, C>0$ such that

$$
|a(z)| \leq C e^{\tau \omega(z)} \quad \forall z \in \mathbb{R}^{2 d}
$$

Then

$$
L_{\psi, \gamma}^{a}: \mathcal{S}_{\omega}\left(\mathbb{R}^{d}\right) \rightarrow \mathcal{S}_{\omega}\left(\mathbb{R}^{d}\right)
$$

and

$$
L_{\psi, \gamma}^{a}: \mathcal{S}_{\omega}^{\prime}\left(\mathbb{R}^{d}\right) \rightarrow \mathcal{S}_{\omega}^{\prime}\left(\mathbb{R}^{d}\right)
$$

are continuous.

Proof. Let $f \in \mathcal{S}_{\omega}\left(\mathbb{R}^{d}\right)$. From Theorem 2.7 we have that for every $\lambda, \rho>0$ there exists $C_{\lambda}>0$ such that

$$
e^{\rho \omega(z)}|a(z)|\left|V_{\psi} f(z)\right| \leq C_{\lambda} e^{(\rho+\tau-\lambda) \omega(z)},
$$

and so, choosing $\lambda \geq \rho+\tau$, we have that $a \cdot V_{\psi} f \in L_{m_{\rho}}^{\infty}\left(\mathbb{R}^{2 d}\right)$ for every $\rho>0$, where $m_{\rho}$ is defined by (3.10). From Proposition 3.7 and (4.34), we have that $L_{\psi, \gamma}^{a} f \in M_{m_{\rho}}^{\infty}\left(\mathbb{R}^{d}\right)$ for every $\rho>0$, and then, from Remark 3.6, $L_{\psi, \gamma}^{a} f \in \mathcal{S}_{\omega}\left(\mathbb{R}^{d}\right)$. To prove the continuity of $L_{\psi, \gamma}^{a}$ on $\mathcal{S}_{\omega}\left(\mathbb{R}^{d}\right)$ we fix $\varphi \in \mathcal{S}_{\omega}\left(\mathbb{R}^{d}\right) \backslash\{0\}, \rho>0$, and we observe that from (3.14) (with $p=q=\infty$ ) and (4.35) we get

$$
\begin{aligned}
\sup _{z \in \mathbb{R}^{2 d}}\left|V_{\varphi}\left(L_{\psi, \gamma}^{a} f\right)(z)\right| e^{\rho \omega(z)} & =\sup _{z \in \mathbb{R}^{2 d}}\left|V_{\varphi} V_{\gamma}^{*}\left(a \cdot V_{\psi} f\right)\right| e^{\rho \omega(z)} \\
& \leq C\left\|V_{\varphi} \gamma\right\|_{L_{v_{\rho}}^{1}} \sup _{z \in \mathbb{R}^{2 d}}\left|a(z) V_{\psi} f(z)\right| e^{\rho \omega(z)} \\
& \leq C^{\prime} \sup _{z \in \mathbb{R}^{2 d}}\left|V_{\psi} f(z)\right| e^{(\tau+\rho) \omega(z)} .
\end{aligned}
$$

From Proposition 2.10 we have that (4.36) is continuous.

Let now $f \in \mathcal{S}_{\omega}^{\prime}\left(\mathbb{R}^{d}\right)$. From Remark 3.6 there exists $\lambda<0$ such that $f \in \boldsymbol{M}_{m_{\lambda}}^{\infty}\left(\mathbb{R}^{d}\right)$; then, choosing $\rho=-|\tau|-|\lambda|$ we have

$$
e^{\rho \omega(z)}|a(z)|\left|V_{\psi} f(z)\right| \leq C e^{(\rho+\tau-\lambda) \omega(z)}<+\infty
$$

for every $z \in \mathbb{R}^{2 d}$, so $a \cdot V_{\psi} f \in L_{m_{\rho}}^{\infty}\left(\mathbb{R}^{2 d}\right)$. Then by Proposition 3.7 we have $L_{\psi, \gamma}^{a} f \in M_{m_{\rho}}^{\infty}\left(\mathbb{R}^{d}\right)$, and from Remark 3.6 we finally have $L_{\psi, \gamma}^{a} f \in \mathcal{S}_{\omega}^{\prime}\left(\mathbb{R}^{d}\right)$. Observe now that for every $u \in \mathcal{S}_{\omega}^{\prime}\left(\mathbb{R}^{d}\right)$ and $v \in \mathcal{S}_{\omega}\left(\mathbb{R}^{d}\right)$ we have

$$
\left\langle L_{\psi, \gamma}^{a} u, v\right\rangle=\left\langle V_{\gamma}^{*}\left(a \cdot V_{\psi} u\right), v\right\rangle=\left\langle u, V_{\psi}^{*}\left(\bar{a} \cdot V_{\gamma} v\right)\right\rangle=\left\langle u, L_{\gamma, \psi}^{\bar{a}} v\right\rangle .
$$

Then $L_{\psi, \gamma}^{a}=\left(L_{\gamma, \psi}^{\bar{a}}\right)^{*}$; since $\bar{a}$ satisfies the same estimates as $a$, the continuity of (4.37) follows from that of (4.36). 
Theorem 4.15. Let $\psi, \gamma \in \mathcal{S}_{\omega}\left(\mathbb{R}^{d}\right) \backslash\{0\}$, and let a be a symbol satisfying (4.35). Then for every $u \in \mathcal{S}_{\omega}^{\prime}\left(\mathbb{R}^{d}\right)$ we have

$$
\mathrm{WF}_{\omega}^{\prime}\left(L_{\psi, \gamma}^{a} u\right) \subseteq \mathrm{WF}_{\omega}^{\prime}(u) .
$$

Proof. Let $z_{0} \notin \mathrm{WF}_{\omega}^{\prime}(u), z_{0} \in \mathbb{R}^{2 d} \backslash\{0\}$. Then there exists an open conic set $\Gamma \subseteq \mathbb{R}^{2 d} \backslash\{0\}$ containing $z_{0}$ such that

$$
\sup _{z \in \Gamma} e^{\lambda \omega(z)}\left|V_{\psi} u(z)\right|<+\infty \quad \forall \lambda>0 .
$$

From (4.35), since $\lambda$ is arbitrary we have

$$
\sup _{z \in \Gamma} e^{\lambda \omega(z)}\left|a(z) V_{\psi} u(z)\right|<+\infty \quad \forall \lambda>0 .
$$

For window functions $\varphi, \gamma \in \mathcal{S}_{\omega}\left(\mathbb{R}^{d}\right)$ we can then repeat the same procedure used in the proof of Proposition 3.2. First, we observe that from the definition of localization operator

$$
V_{\varphi}\left(L_{\psi, \gamma}^{a} u\right)=V_{\varphi} V_{\gamma}^{*}\left(a \cdot V_{\psi} u\right) .
$$

Now, it is not difficult to see that

$$
\begin{aligned}
& V_{\varphi}\left(L_{\psi, \gamma}^{a} u\right)(x, \xi)=\int_{\mathbb{R}^{2 d}}\left(a \cdot V_{\psi} u\right)(s, \eta) \overline{V_{\gamma}(\Pi(z) \varphi)}(s, \eta) d s d \eta, \\
& \overline{V_{\gamma}(\Pi(z) \varphi)}(s, \eta)=V_{\varphi} \gamma(x-s, \xi-\eta) e^{-i\langle s, \xi-\eta\rangle},
\end{aligned}
$$

and hence

$$
\left|V_{\varphi}\left(L_{\psi, \gamma}^{a} u\right)\right| \leq\left|a \cdot V_{\psi} u\right| *\left|V_{\varphi} \gamma\right| .
$$

Consequently, for every open conic set $\Gamma^{\prime} \subseteq \mathbb{R}^{2 d} \backslash\{0\}$ containing $z_{0}$ and such that $\overline{\Gamma^{\prime} \cap S_{2 d-1}} \subseteq \Gamma$ we have (see the proof of Proposition 3.2)

$$
\sup _{z \in \Gamma^{\prime}} e^{\lambda \omega(z)}\left|V_{\varphi}\left(L_{\psi, \gamma}^{a} u\right)(z)\right|<+\infty, \quad \lambda>0 .
$$

This implies that $z_{0} \notin \mathrm{WF}_{\omega}^{\prime}\left(L_{\psi, \gamma}^{a} u\right)$ and the proof is complete.

\section{Examples}

In this section we compute the Gabor wave front set for some particular $u \in \mathcal{S}_{\omega}^{\prime}\left(\mathbb{R}^{d}\right.$ ) (see also the examples in [35]).

Example 5.1. Consider the Dirac distribution $u=\delta \in \mathcal{S}_{\omega}^{\prime}\left(\mathbb{R}^{d}\right)$ for every weight $\omega$. We have that

$$
V_{\varphi} \delta(x, \xi)=\overline{\varphi(-x)}
$$

Since $V_{\varphi} \delta(0, \xi)=\overline{\varphi(0)}$, choosing $\varphi$ in such a way that $\varphi(0) \neq 0$ we have

$$
\{0\} \times\left(\mathbb{R}^{d} \backslash\{0\}\right) \subseteq \mathrm{WF}_{\omega}^{\prime}(\delta) .
$$

Let now $\left(x_{0}, \xi_{0}\right) \in \mathbb{R}^{2 d} \backslash\{0\}$ such that $x_{0} \neq 0$, and consider an open conic set containing $\left(x_{0}, \xi_{0}\right)$ of the form

$$
\Gamma=\left\{(x, \xi) \in \mathbb{R}^{2 d} \backslash\{0\}:|\xi|<C|x|\right\}
$$


for $C>0$. From the subadditivity of $\omega$, there exists $C_{1}>0$ such that, writing $z=(x, \xi)$,

$$
\sup _{z \in \Gamma} e^{\lambda \omega(z)}\left|V_{\varphi} \delta(z)\right| \leq \sup _{x \in \mathbb{R}^{d}} e^{\lambda C_{1} \omega(x)}|\varphi(-x)|<+\infty
$$

since $\varphi \in \mathcal{S}_{\omega}\left(\mathbb{R}^{d}\right)$. Then $\left(x_{0}, \xi_{0}\right) \notin \mathrm{WF}_{\omega}^{\prime}(\delta)$, and so $\mathrm{WF}_{\omega}^{\prime}(\delta)=\{0\} \times\left(\mathbb{R}^{d} \backslash\{0\}\right)$. From Proposition 3.19 we have that for every $\bar{x} \in \mathbb{R}^{d}$, writing $\delta_{\bar{x}}$ for the Dirac distribution centered at $\bar{x}$

$$
\mathrm{WF}_{\omega}^{\prime}\left(\delta_{\bar{x}}\right)=\{0\} \times\left(\mathbb{R}^{d} \backslash\{0\}\right)
$$

Example 5.2. Let $u=1$ be the function identically 1, that belong to $\mathcal{S}_{\omega}^{\prime}\left(\mathbb{R}^{d}\right)$ for every weight $\omega$. A direct computation shows that

$$
V_{\varphi}(\mathbf{1})=e^{-i\langle x, \xi\rangle} \overline{\hat{\varphi}(-\xi)}
$$

since $\hat{\varphi} \in \mathcal{S}_{\omega}\left(\mathbb{R}^{d}\right)$ we can proceed as in Example 5.1, obtaining that for every weight $\omega$, $\mathrm{WF}_{\omega}^{\prime}(\mathbf{1})=\left(\mathbb{R}^{d} \backslash\{0\}\right) \times\{0\}$. From Proposition 3.19 we then have that for every $\bar{\xi} \in \mathbb{R}^{d}$ and for every weight $\omega$,

$$
\mathrm{WF}_{\omega}^{\prime}\left(e^{i\langle\cdot \bar{\xi}\rangle}\right)=\left(\mathbb{R}^{d} \backslash\{0\}\right) \times\{0\}
$$

Example 5.3. We consider now the function $u(x)=e^{i c x^{2} / 2}$, for $x \in \mathbb{R}$ and $c \in \mathbb{R} \backslash\{0\}$. Observe that $u \in \mathcal{S}_{\omega}^{\prime}(\mathbb{R})$ for every $\omega$. Choosing as window function the Gaussian $\varphi(t)=e^{-t^{2} / 2}$, that belongs to $\mathcal{S}_{\omega}(\mathbb{R})$ for every $\omega$, we have, as in Example 6.6 of [35], that there exists $C>0$ such that

$$
\left|V_{\varphi} u(x, \xi)\right|=C \exp \left(-\frac{(\xi-c x)^{2}}{2\left(1+c^{2}\right)}\right) .
$$

Then, proceeding in a similar way as in the previous cases we have

$$
\mathrm{WF}_{\omega}^{\prime}(u)=\{(x, c x): x \in \mathbb{R} \backslash\{0\}\}
$$

for every weight $\omega$.

We observe that in the cases (5.1) and (5.2) the Gabor wave front set gives rougher information since it does not take into account translations and modulations, while for the case (5.3) it gives finer information, since it identifies the so-called instantaneous frequency, that is the only direction along which the time-frequency content of $u$ does not decay. For a comparison of the Gabor wave front set of the element considered in the previous examples with other type of global wave front set (at least in the frame of tempered distributions) we refer to [35].

We observe now that in the previous examples the considered distributions have the same wave front set for every weight $\omega$. In general the Gabor wave front set may depend on $\omega$, as shown in the next example.

Example 5.4. Let $\omega$ and $\sigma$ be two weight functions, such that $\omega(t) \leq \sigma(t)$ and $\mathcal{S}_{\sigma}\left(\mathbb{R}^{d}\right) \cap$ $\mathcal{D}\left(\mathbb{R}^{d}\right) \subsetneq \mathcal{S}_{\omega}\left(\mathbb{R}^{d}\right) \cap \mathcal{D}\left(\mathbb{R}^{d}\right)$. We then fix a function $f \in \mathcal{S}_{\omega}\left(\mathbb{R}^{d}\right)$ with compact support such that $f \notin \mathcal{S}_{\sigma}\left(\mathbb{R}^{d}\right)$. From Proposition 3.18 we have

$$
\mathrm{WF}_{\omega}^{\prime}(f)=\emptyset \text {. }
$$

Fix now a window $\varphi_{0} \in \mathcal{S}_{\sigma}\left(\mathbb{R}^{d}\right)$ with compact support such that $\varphi_{0} \equiv 1$ on $\operatorname{supp}(f)$. From the definition of short-time Fourier transform, we then have that the orthogonal projection on $\mathbb{R}_{x}^{d}$ 
of the support of $V_{\varphi_{0}} f(x, \xi)$ is compact. Let now $z_{0}=\left(x_{0}, \xi_{0}\right) \in \mathbb{R}^{2 d}$ with $x_{0} \neq 0$, and fix an open conic set containing $z_{0}$ of the form

$$
\Gamma=\left\{(x, \xi) \in \mathbb{R}^{2 d} \backslash\{0\}:|\xi|<C|x|\right\},
$$

for $C>0$. We then have that $\overline{\Gamma \cap \operatorname{supp}\left(V_{\varphi_{0}} f\right)}$ is compact, so the condition (3.1) is satisfied for every $\lambda>0$. Then $\left(x_{0}, \xi_{0}\right) \notin \mathrm{WF}_{\sigma}^{\prime}(f)$ for every $x_{0} \neq 0$. Consider now a point of the type $\left(0, \xi_{0}\right)$ with $\xi_{0} \neq 0, \xi_{0} \in \mathbb{R}^{d}$. From the fact that $\varphi_{0} \equiv 1$ on $\operatorname{supp}(f)$, we have

$$
V_{\varphi_{0}} f(0, \xi)=\int e^{-i\langle t, \xi\rangle} f(t) \overline{\varphi_{0}(t)} d t=\hat{f}(\xi) .
$$

Since $f \notin \mathcal{S}_{\sigma}\left(\mathbb{R}^{d}\right)$, we have that there exists $\lambda>0$ such that

$$
\sup _{\xi \in \mathbb{R}^{d}} e^{\lambda \sigma(\xi)}\left|V_{\varphi_{0}} f(0, \xi)\right|=+\infty,
$$

so (3.1) cannot be satisfied in an open conic set containing $\left(0, \xi_{0}\right)$, and then $\left(0, \xi_{0}\right) \in \mathrm{WF}_{\omega}^{\prime}(f)$. We then have that

$$
\mathrm{WF}_{\sigma}^{\prime}(f)=\{0\} \times\left(\mathbb{R}^{d} \backslash\{0\}\right) ;
$$

in particular $\mathrm{WF}_{\sigma}^{\prime}(f) \neq \mathrm{WF}_{\omega}^{\prime}(f)$.

Acknowledgments. The authors are very grateful to the reviewers for the careful reading and for the comments and remarks which improve the presentation and the quality of the paper.

The authors were partially supported by the INdAM-Gnampa Project 2016 "Nuove prospettive nell'analisi microlocale e tempo-frequenza", by FAR 2013 (University of Ferrara) and by the project "Ricerca Locale - Analisi di Gabor, operatori pseudodifferenziali ed equazioni differenziali" (University of Torino). The research of the second author was partially supported by the project MTM2016-76647-P.

\section{References}

[1] A. Albanese, D. Jornet, A. Oliaro, Quasianalytic wave front sets for solutions of linear partial differential operators, Integr. Equ. Oper. Theory 66 (2010), 153-181.

[2] A. Albanese, D. Jornet, A. Oliaro, Wave front sets for ultradistribution solutions of linear partial differential operators with coefficients in non-quasianalytic classes, Math. Nachr. 285, n. 4 (2012), 411-425.

[3] G. Björck, Linear partial differential operators and generalized distributions, Ark. Mat. 6, n. 21 (1966), 351-407.

[4] C. Boiti, E. Gallucci, The overdetermined Cauchy problem for $\omega$-ultradifferentiable functions, Manuscripta Math. 155, n.3-4 (2018), 419-448.

[5] C. Boiti, D. Jornet, A simple proof of Kotake-Narasimhan Theorem in some classes of ultradifferentiable functions, J. Pseudo-Differ. Oper. Appl. 8(2), (2017), 297-317.

[6] C. Boiti, D. Jornet, A characterization of the wave front set defined by the iterates of an operator with constant coefficients, Rev. R. Acad. Cienc. Exactas Fs. Nat. Ser. A Math. RACSAM 111 (2017), no. 3, 891-919.

[7] C. Boiti, D. Jornet, J. Juan-Huguet, Wave front sets with respect to the iterates of an operator with constant coefficients, Abstr. Appl. Anal., (2014), Art. ID 438716, 1-17; http://dx.doi.org/10.1155/2014/438716 
[8] C. Boiti, D. Jornet, A. Oliaro, Regularity of partial differential operators in ultradifferentiable spaces and Wigner type transforms, J. Math. Anal. Appl. 446 (2017), 920-944.

[9] J. Bonet, R. Meise, S.N. Melikhov, A comparison of two different ways to define classes of ultradifferentiable functions, Bull. Belg. Math. Soc. Simon Stevin, Volume 14, Number 3 (2007), 425-444.

[10] J.M. Borwein, A.S. Lewis, Convex analysis and nonlinear optimization. Theory and examples, CMS Books in Mathematics/Ouvrages de Mathématiques de la SMC, 3, Springer, New York, 2006.

[11] R.W. Braun, R. Meise, B.A. Taylor, Ultradifferentiable functions and Fourier analysis, Result. Math. 17 (1990), 206-237.

[12] M. Cappiello, R. Schulz, Microlocal analysis of quasianalytic Gelfand-Shilov type ultradistributions, Complex Var. Elliptic Equ. 61, n. 4 (2016), 538-561.

[13] E. Carypis, P. Wahlberg, Propagation of Exponential Phase Space Singularities for Schrödinger Equations with Quadratic Hamiltonians, J. Fourier Anal. Appl. 23, n. 3 (2017), 530-571.

[14] O. Christensen, An introduction to frames and Riesz bases, Applied and Numerical Harmonic Analysis, Birkhäuser/Springer, 2016.

[15] C. Fernández, A. Galbis, D. Jornet, Pseudodifferential operators on non-quasianalytic classes of Beurling type, Studia Math. 167, n. 2 (2005), 99-131.

[16] C. Fernández, A. Galbis, D. Jornet, Pseudodifferential operators of Beurling type and the wave front set, J. Math. Anal. Appl. 340 (2008), no. 2, 1153-1170.

[17] C. Fieker, P-Konvexität und $\omega$-Hypoelliptizität für partielle Differentialoperatoren mit konstanten Koeffizienten, Diplomarbeit, Mathematischen Institut der Heinrich-Heine-Universität Düsseldorf (1993).

[18] K. Gröchenig, Foundations of Time-Frequency Analysis, Birkhäuser, Boston (2001).

[19] K. Gröchenig, G. Zimmermann, Spaces of Test Functions via the STFT, J. Funct. Spaces Appl. 2, n. 1 (2004), 25-53

[20] C. Heil, A basis theory primer, Applied and Numerical Harmonic Analysis, Birkhäuser/Springer, New York, 2011.

[21] L. Hörmander, Fourier integral operators, Acta Math. 127, n. 1 (1971), 79-183.

[22] L. Hörmander, Quadratic Hyperbolic operators. In Cattabriga, L., Rodino, L. (eds.) Microlocal Analysis and Applications. Lecture Notes in Mathematics, vol. 1495, pp. 118-160. Springer, Berlin (1991).

[23] L. Hörmander, The analysis of linear partial differential operators, vol. I, Springer-Verlag, Berlin (1983)

[24] L. Hörmander, The analysis of linear partial differential operators, vol. II, Springer-Verlag, Berlin (1983)

[25] L. Hörmander, The analysis of linear partial differential operators, vol. III, Springer-Verlag, Berlin (1985)

[26] A.J.E.M. Janssen, Duality and Biorthogonality for Weyl-Heisenberg Frames, J. Fourier Anal. Appl. 1, n. 4 (1995), 403-436.

[27] M. Langenbruch, Hermite functions and weighted spaces of generalized functions, Manuscripta Math. 119, n. 3 (2006), 269-285.

[28] R. Meise, D. Vogt, Introduction to Functional Analysis, Oxford Science Publications, Clarendon Press, Oxford, 1997.

[29] S. Nakamura, Propagation of the homogeneous wave front set for Schrödinger equations, Duke Math. J. 126 (2005), 349-367.

[30] F. Nicola, L. Rodino, Global Pseudo-Differential Calculus on Euclidean Spaces, Springer, Basel (2010).

[31] S. Pilipović, Tempered ultradistributions, Boll. U.M.I. B (7) 2 (1988), no. 2, 235-251.

[32] B. Prangoski, Pseudodifferential operators of infinite order in spaces of tempered ultradistributions, J. Pseudo-Differ. Oper. Appl. 4 (2013), no. 4, 495-549.

[33] S. Pilipović, B. Prangoski, Anti-Wick and Weyl quantization on ultradistribution spaces, J. Math. Pures Appl. (9) 103 (2015), no. 2, 472-503.

[34] L. Rodino, Linear partial differential operators and Gevrey spaces, World Scientific Publishing Co., Inc. River Edge, NJ, (1993).

[35] L. Rodino, P. Wahlberg, The Gabor wave front set, Monatsh. Math. 173 (2014), 625-655.

[36] R. Schulz, P. Wahlberg, Microlocal properties of Shubin pseudodifferential and localization operators, J. Pseudo-Differ. Oper. Appl. 7, n. 1 (2016), 91-111.

[37] R. Schulz, P. Wahlberg, Equality of the homogeneous and the Gabor wave front set, Comm. Partial Differential Equations 42, n. 5 (2017), 703-730. 
[38] M.A. Shubin, Pseudodifferential Operators and Spectral Theory, Springer-Verlag, Berlin, 1987.

[39] J. Sjöstrand, Singularités analytiques microlocales, Astérisque 95(1982), 1-166.

[40] J. Toft, The Bargmann transform on modulation and Gelfand-Shilov spaces, with applications to Toeplitz and pseudo-differential operators, J. Pseudo-Differ. Oper. Appl. 3, n.2 (2012), 145-227.

[41] J. Toft, Images of function and distribution spaces under the Bargmann transform, J. Pseudo-Differ. Oper. Appl. 8, n.1 (2017), 83-139.

[42] F. Treves, Topological vector spaces, distributions and kernels, Academic Press, New York, 1967.

Dipartimento di Matematica e Informatica, Università di Ferrara, Via Machiavelli n. 30, I-44121 FERRARA, ITALY

E-mail address: chiara.boiti@unife.it

Instituto Universitario de Matemática Pura y Aplicada IUMPA, Universitat Politècnica de València, Camino de Vera, S/n, E-46071 Valencia, Spain

E-mail address: djornet@mat.upv.es

Dipartimento di Matematica, Università di Torino, Via Carlo Alberto n. 10, I-10123 Torino, ITALY

E-mail address: alessandro.oliaro@unito.it 